\title{
Article
}

\section{The Automaticity of Semantic Processing Revisited: Auditory Distraction by a Categorical Deviation}

Vachon, Francois, Marsh, John Everett and Labonte, Katherine Available at https://clok.uclan.ac.uk/30122/

Vachon, Francois, Marsh, John Everett orcid iconORCID: 0000-0002-9494-1287 and Labonte, Katherine (2019) The Automaticity of Semantic Processing Revisited: Auditory Distraction by a Categorical Deviation. Journal of Experimental Psychology: General, 149 (7). pp. 1360-1397. ISSN 0096-3445

It is advisable to refer to the publisher's version if you intend to cite from the work. http://dx.doi.org/10.1037/xge0000714

For more information about UCLan's research in this area go to http://www.uclan.ac.uk/researchgroups/ and search for < name of research Group>.

For information about Research generally at UCLan please go to http://www.uclan.ac.uk/research/

All outputs in CLoK are protected by Intellectual Property Rights law, including Copyright law. Copyright, IPR and Moral Rights for the works on this site are retained by the individual authors and/or other copyright owners. Terms and conditions for use of this material are defined in the policies page.

\section{CLoK}

Central Lancashire online Knowledge www.clok.uclan.ac.uk

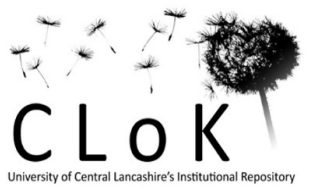


Revision of XGE-2018-1119R2 as invited by the action editor, Moshe Naveh-Benjamin

The Automaticity of Semantic Processing Revisited: Auditory Distraction by a Categorical

Deviation

François Vachon $^{1,2}$, John E. Marsh ${ }^{2,3}$, \& Katherine Labonté ${ }^{1}$

${ }^{1}$ Université Laval, Québec, Canada

${ }^{2}$ University of Gävle, Gävle, Sweden

${ }^{3}$ University of Central Lancashire, Preston, UK

Word count (main text + abstract): 23,692

\section{Author Note}

We are grateful to Valérie Gagnon, Karianne Guay, Joanie Lamirande, Maxime Legendre, Michaël Lévesque-Dion, Alessandro Pozzi, and Rosalie Savard for running the experiments. Thanks are also due to Neil McLatchie for his guidance regarding the Bayes factor metaanalysis, and to Hae-Sung Jeon for her advice on the acoustical analysis of our spoken stimuli. This research was supported by a grant (418623-2013) from the Natural Sciences and Engineering Research Council of Canada (NSERC) awarded to François Vachon and a grant from the Swedish Research Council (2015-01116) awarded to Patrik Sörqvist and John E. Marsh. Katherine Labonté received support from NSERC in the form of a doctoral scholarship. Portions of this research were reported at the $27^{\text {th }}$ Association for Psychological Science Annual Convention, May 2015, New York, NY, at the $57^{\text {th }}$ Annual Meeting of the Psychonomic Society, November 2016, Boston, MA, and at the $60^{\text {th }}$ Annual Meeting of the Psychonomic Society, November 2019, Montreal, Quebec, Canada. Correspondence concerning this article may be addressed to François Vachon, École de psychologie, Université Laval, Québec (QC), G1V 0A6, Canada.E-mail: francois.vachon@psy.ulaval.ca. 
Revision of XGE-2018-1119R2 as invited by the action editor, Moshe Naveh-Benjamin

The Automaticity of Semantic Processing Revisited: Auditory Distraction by a Categorical

Deviation

François Vachon ${ }^{1,2}$, John E. Marsh ${ }^{2,3}$, \& Katherine Labonté ${ }^{1}$

${ }^{1}$ Université Laval, Québec, Canada

${ }^{2}$ University of Gävle, Gävle, Sweden

${ }^{3}$ University of Central Lancashire, Preston, UK

Word count (main text + abstract): 23,692

\section{Author Note}

We are grateful to Valérie Gagnon, Karianne Guay, Joanie Lamirande, Maxime Legendre, Michaël Lévesque-Dion, Alessandro Pozzi, and Rosalie Savard for running the experiments. Thanks are also due to Neil McLatchie for his guidance regarding the Bayes factor metaanalysis, and to Hae-Sung Jeon for her advice on the acoustical analysis of our spoken stimuli. This research was supported by a grant (418623-2013) from the Natural Sciences and Engineering Research Council of Canada (NSERC) awarded to François Vachon and a grant from the Swedish Research Council (2015-01116) awarded to Patrik Sörqvist and John E. Marsh. Katherine Labonté received support from NSERC in the form of a doctoral scholarship. Portions of this research were reported at the $27^{\text {th }}$ Association for Psychological Science Annual Convention, May 2015, New York, NY, at the $57^{\text {th }}$ Annual Meeting of the Psychonomic Society, November 2016, Boston, MA, and at the $60^{\text {th }}$ Annual Meeting of the Psychonomic Society, November 2019, Montreal, Quebec, Canada. Correspondence concerning this article may be addressed to François Vachon, École de psychologie, Université Laval, Québec (QC), G1V 0A6, Canada.E-mail: francois.vachon@psy.ulaval.ca. 


\begin{abstract}
Automatic information processing has been and still is a debated topic. Traditionally, automatic processes are deemed to take place autonomously and independently of top-down cognitive control. For decades, the literature on reading has brought to the fore empirical phenomena such as Stroop and semantic priming effects that provide support for the assumption that semantic information can be accessed automatically. More recently, there has been growing evidence that semantic processing is in fact susceptible to higher level cognitive influences, suggesting that this form of processing is instead conditionally automatic. The purpose of the present study was to revisit this debate using a novel approach: the automatic access to the meaning of irrelevant auditory stimuli was tested through the assessment of their distractive power. More specifically, we aimed to examine whether a categorical change in the content of to-be-ignored auditory sequences composed of speech items that are personally non-significant to participants (e.g., a digit among letters) can disrupt an unrelated visual focal task. In seven experiments, we assessed this categorical deviation effect and its functional properties. We established that distraction by categorical deviation is non-contingent on the activated task set and appears resistant to topdown control manipulations. By suggesting not only that the semantic content of the irrelevant sound can be extracted preattentively, but also that such semantic activation is ineluctable during auditory distraction, these findings shed new light on the automatic nature of semantic processing.
\end{abstract}

Keyword: semantic processing; automaticity; attentional capture; deviation effect; irrelevant sound 


\section{The Automaticity of Semantic Processing Revisited: Auditory Distraction by a Categorical}

\section{Deviation}

Ever since the early days of scientific psychology automatic information processing has been a hotly debated topic. Automatic processes are traditionally deemed to take place autonomously and independently of top-down control. Hence, early theories of automaticity (e.g., Posner \& Snyder, 1975; Schneider \& Shiffrin, 1977) hold that an automatic cognitive process must be independent of capacity-limited attentional resources, can function in parallel with other processes without interference, can occur unconsciously, and cannot be prevented. One key premise of this classical view of automaticity is the independence of an automatic process from top-down influences such as attention, task (or attentional) sets, task demands, and intention or behavioral goals. Any process that is contingent on the way the cognitive system is configured is said to be nonautomatic (cf. Logan, 1988). From this classical all-or-none standpoint, top-down control is confined to the domain of conscious cognition. Given that decades of research on human cognition have revealed that most cognitive processes can be more or less susceptible to attentional factors, and hence be considered as nonautomatic, such an inflexible conceptualization of automaticity is not really helpful in determining whether a process is automatic or not (cf. Kiefer \& Martens, 2010). Moreover, the existence of various automatic processes would place considerable demands on cognitive control given their likely influence on conscious processing (e.g., Botvinick, Braver, Barch, Carter, \& Cohen, 2001).

Although this all-or-none view remains influential nowadays, at least implicitly, several theoretical accounts of automaticity have been elaborated since (see Moors \& De Houwer, 2006, 2007). Most of these contemporary approaches to automaticity tend to offer more flexibility in the conception of automatic processing (e.g., Ansorge, Kunde, \& Kiefer, 2014; Bargh, 1989, 1992; Kiefer \& Martens, 2010; Naccache, Blandin, \& Dehaene, 2002; Neumann, 1984, 1990). 
Challenging the classical view, these refined theories of automaticity share the idea that an automatic process is triggered unconsciously, but nevertheless depends on the current configuration of the cognitive system. Thus, this approach assumes that automatic processes can be under the control of higher-level cognitive influences. This dependency on currently active task representations is often referred to as conditional automaticity (Bargh, 1989, 1992; Hackley, 1993; Kahneman \& Treisman, 1984; Logan, 1989; Woldorff, Hackley, \& Hillyard, 1991). In line with this refined view of automaticity, Moors and De Houwer $(2006,2007)$ proposed a set of key criteria, called features, to investigate in order to determine the degree of automaticity of a process. These so-called goal-related features include (un)intentional, goal(in)dependent, (un)controllable, and autonomous. A process is intentional when it is caused by an intention, i.e. the goal representation of that process, whereas an unintentional process is not produced by the goal to engage in the process. A goal-dependent process is one that depends on a goal that is in place. If that goal is the proximal goal to engage in the process, then the goaldependent process is also considered as intentional. Hence, one can view an intentional process as a form of goal-dependent process. A process is considered to be goal-independent when it does not depend on a goal (proximal or remote) for its occurrence. Hence, goal-independent processes are by definition a subtype of unintentional processes. A process is controllable when it is possible for the individual to control it whereas an uncontrollable process is one that it is impossible for the person to control. Finally, an autonomous process is one that is not controlled by outside forces. In contrast with an uncontrolled process, which is specified in terms of a processing goal, a fully autonomous process is uncontrolled in the sense of every possible goal. Table 1 summarizes the characteristics of each type of (non)automaticity feature according to Moors and De Houwer's nomenclature. It is noteworthy that these goal-related features are not perfectly orthogonal as there is some conceptual overlap among them. In order to diagnose the 
automatic nature of processes, the authors recommend investigating these features separately. To be considered as fully automatic, a process must be at the same time unintentional, goalindependent, uncontrollable, and autonomous.

Table 1

Characteristics of the main (non)automaticity features according to Moors and De Houwer (2006, 2007).

\begin{tabular}{lll}
\hline \multicolumn{1}{c}{ Feature } & \multicolumn{1}{c}{ Condition } & \multicolumn{1}{c}{ Effect } \\
\hline Intentional & Intention present & Process present \\
Unintentional & Intention absent & Process present \\
\hline Goal-dependent & Goal present & Process present \\
Goal-independent & Goal absent & Process present \\
\hline Controllable & Goal pertaining to process present & Effect on process \\
& - To engage in process & - Occurrence of process \\
& - To alter process & - Change in process \\
& - To stop process & - Interruption of process \\
& - To avoid process & - Prevent of process \\
Uncontrollable & Goal pertaining to process absent or present & No effect on process \\
\hline Autonomous & Goal pertaining to process absent or present & Process present \\
& & No effect on process \\
\hline
\end{tabular}

An important question in the study of language is to what degree semantic processes are automatic or nonautomatic. For some time, several researchers favored the view that semantic processing can occur in a purely automatic fashion. Yet, more recent demonstrations that semantic processing can be susceptible to top-down control have led others to conclude that it would be in fact conditionally automatic. Adopting Moors and De Houwer's $(2006,2007)$ feature approach, the present study revisits this debate about the automaticity of semantic processing by exploiting a novel experimental paradigm inspired from research in the field of auditory distraction. 


\section{Automaticity of Semantic Processing}

The literature on reading provides prima facie evidence supporting the assumption that semantic processing is fully automatic (i.e. according to the standards of the classical approach to automaticity), the so-called Stroop effect being the example par excellence (see MacLeod, 1991, for a review). When naming the color of the ink in which a color word is printed, naming latencies tend to be longer for incongruent stimuli (e.g., the word 'red' printed in green) compared to congruent stimuli (e.g., the word 'red' printed in red). These increased latencies are assumed to reflect the automatic activation of the semantic information conveyed by the printed word, rendering that information unavoidable to the reader, which, in turn, interferes with the deliberate report of conflicting information, namely the color of the ink. Another phenomenon arguing in favor of the automaticity of semantic processing is semantic priming (see J. H. Neely, 1991, for a review). The fact that the semantic content of a subliminal—i.e. masked—stimulus (the prime) can affect the perception of a subsequent stimulus (the target) has been taken as evidence that semantic processing can take place without awareness (e.g., Dehaene et al., 1998; Kiefer, 2002; Marcel, 1983) and even without attention (e.g., Luck, Vogel, \& Shapiro, 1996; Maki, Frigen, \& Paulson, 1997). More recently, Schnuerch, Kreitz, Gibbons, and Memmert (2016) showed that stimuli remaining unnoticed due to inattentional blindness nevertheless interfere with the processing of semantically-related attended objects, providing further evidence that the meaning of unconscious stimuli can be accessed instantaneously.

Yet, there is growing evidence that semantic processing can be under the control of higher-level cognitive influences. In fact, the impact of unconscious semantic information appears crucially dependent upon top-down attention, intentions, and task sets (e.g., Kiefer \& Martens, 2010; Kunde, Kiesel, \& Hoffmann, 2003; Neumann, 1984). For instance, a task that directs attention to non-semantic properties of the priming stimulus (e.g., focusing on a letter 
rather than on the whole word) tends to attenuate or even eliminate semantic priming (see Maxfield, 1997) and the Stroop effect (e.g., Besner, Stolz, \& Boutilier, 1997; but see J. H. Neely \& Kahan, 2001). Similarly, the need to perform a perceptual judgment on the prime strongly diminishes the semantic priming of a target compared to a semantic judgment (e.g., Kiefer \& Martens, 2010; Vachon \& Jolicœur, 2011). The abolition of semantic priming is not limited to cases for which no semantic processing of the prime is required, as the same effects are found as long as different task sets are applied to the prime and the target (e.g., Logan \& Schulkind, 2000; Tse \& J. H. Neely, 2007; Vachon, Tremblay, \& Jones, 2007). This is true even for situations in which a distinct semantic judgment is required for both the prime and the target tasks (Vachon \& Jolicœur, 2012). Such findings strongly suggest that semantic activation is contingent on how the task set is configured (e.g., Kiefer, 2007; Kiefer \& Martens, 2010; Naccache et al., 2002; Vachon \& Jolicœur, 2012). Accordingly, subliminal stimuli bias subsequent processing to the degree they match pre-specified, goal-related cognitive templates. If a stimulus (e.g., a digit) sufficiently matches one of these templates (e.g., 'categorize the target digit'), the corresponding activation will then be initiated and spread automatically (e.g., Kunde et al., 2003). Indeed, the power of the semantic preactivation (or priming) of a stimulus has been shown to be very limited when such preactivation is not motivationally relevant for current action goals (Kreitz, Schnuerch, Furley, Gibbons, \& Memmert, 2015).

The fact that semantic processing appears susceptible to top-down control suggests that this type of processing may not be fully automatic after all. Yet, the debate regarding the full or conditional automaticity of semantic processing may have been spawned by the paradigms typically used to study semantic activation. For instance, in the semantic priming technique, assessing the impact of a prime on a target requires the two objects to be related in some way (see, e.g., Dehaene et al., 1998; Luck et al., 1996; Schnuerch et al., 2016). Moreover, the prime, 
either masked or not, is typically presented in the same location as the target so that it appears where attention is focused. Under such conditions, there is a risk that the semantic information conveyed by the prime somehow matches the configuration of the cognitive system established for the appropriate processing of the target. If so, the activated task set would be more likely to exert some influence on—or to sensitize (cf. Kiefer \& Martens, 2010)—-the processing of the prime, to hence reveal top-down effects. The purpose of the present study was to provide an investigation of the automaticity of semantic processing while minimizing these potential influences. To do so, one could examine the semantic activation of stimuli that are irrelevant to the action goals and, thus, must be ignored. One way to determine whether-and to what extent—an irrelevant stimulus has been processed is through the assessment of its distracting power, that is, its impact on the deliberate processing involved in an ongoing task. A cross-modal distraction paradigm is ideal for the purpose of the present study as it allows minimization of the relationship between the goal-related and irrelevant materials. We therefore selected the irrelevant sound paradigm (see Hughes \& Jones, 2003, for a review) because it does not involve focal attention to the auditory stimuli (see Macken, Tremblay, Houghton, Nicholls, \& Jones, 2003). Indeed, participants are instructed to focus exclusively on the task-relevant visual material and ignore any auditory stimulation as they are not going to be tested on its content. Within the context of this paradigm, Röer, Körner, Buchner, and Bell (2017b) showed that irrelevant auditory sequences composed of related category exemplars can facilitate the subsequent production of exemplars from the same category. This finding is promising as it suggests that tobe-ignored (TBI) sounds can be processed at a post-categorical level. In the present study, the automaticity of access to meaning was not assessed through semantic priming; it was instead tested through semantic auditory distraction. More specifically, we aimed to examine whether the semantic content of the irrelevant sound can involuntarily attract attention away from a 
visual, focal task. In such a context, the finding of distraction effects caused by the semantic content of TBI sound would suggest that the activation of that content was unintentional (cf. Moors \& De Houwer, 2006).

\section{Semantic Auditory Distraction}

The early behavioral evidence that individuals can notice their own name occurring in an unattended channel (Moray, 1959) — the so-called cocktail party effect—revealed that some of the content of irrelevant sound can be extracted preattentively. There have been ample reports of semantic auditory distraction effects since then. Yet, the mere demonstration that the semanticity of irrelevant sound can interfere with ongoing cognitive activities cannot be taken as evidence for the full automaticity of semantic processing (Holender, 1986; Lachter, Forster, \& Ruthruff, 2004; Wood \& Cowan, 1995). In fact, existing evidence of semantic auditory distraction remains inconclusive in that regard. For instance, the episodic recall of semantic information (e.g., words drawn from a single semantic category) tends to be disrupted by TBI sound that is similar in semantic content to the to-be-remembered (TBR) visual stimuli (e.g., spoken words drawn from the same semantic category; Beaman, 2004; Marsh, Hughes, \& Jones, 2008, 2009; C. B. Neely \& LeCompte, 1999; but see Hanczakowski, Beaman, \& Jones, 2017 for a beneficial effect of TBRTBI semantic similarity). Although such between-sequence semantic similarity effects point to the obligatory semantic activation of the spoken material, interference from the meaning of the background sound was found exclusively when the focal task involved a semantic component (e.g., Marsh, Hughes, \& Jones, 2008, 2009; Sörqvist, Marsh, \& Jahncke, 2010). This form of distraction is better explained in terms of interference-by-process: the semantic activation of the irrelevant speech competes with—and impairs accessibility to- the semantic representations activated through the deliberate semantic processing of the TBR items (see Marsh \& Jones, 2010, for a discussion). Because of the semantic similarity between the TBR and TBI material, it 
is not clear from this body of research whether access to the meaning of the irrelevant sound was purely automatic or triggered in a goal-dependent fashion. The fact that this form of semantic auditory distraction appears susceptible to top-down cognitive control (Marsh, Sörqvist, Hodgetts, Beaman, \& Jones, 2015; Marsh, Sörqvist, \& Hughes, 2015) provides evidence against the full automaticity hypothesis.

One form of auditory distraction that may be more suitable for the purpose of the present study is attentional capture, the exogenous orienting of the attentional focus away from ongoing mental activity due to the occurrence of task-irrelevant stimulation. Although auditory attentional capture can be contingent on the individual's task set (e.g., Dalton \& Lavie, 2007; Escera, Alho, Winkler, \& Näätänen, 1998; Meade \& Fernandes, 2016), it can also reflect the action of a purely stimulus-driven mechanism (e.g., Dalton \& Lavie, 2004; Vachon, Labonté, \& Marsh, 2017). Notwithstanding the attentional grabbing potential of the acoustical properties of irrelevant sound, there is evidence that the semantic content of unattended sound is also endowed with the power to capture attention. This is particularly the case for highly self-relevant information such as one's own name, which can be consciously detected when presented in a TBI auditory channel (e.g., Moray, 1959; Wood \& Cowan, 1995) and tends to attract attention thereby disrupting performance (e.g., Conway, Cowan, \& Bunting, 2001; Röer, Bell \& Buchner, 2013). This attention-capturing power of personal significance is not restricted to names as it extends to a mother's own baby cries (Formby, 1967), and even to one's personal ringtone (Roye, Jacobsen, \& Schröger, 2007). Task-irrelevant emotionally arousing sounds are also known to capture attention (e.g., Keil et al., 2007; Marsh, Yang et al., 2018; Sokka et al., 2014; Thierry \& Roberts, 2007) and impair prevailing mental activity (e.g., Buchner, Rothermund, Wentura, \& Mehl, 2004; Buchner, Mehl, Rothermund, \& Wentura, 2006; Keil et al., 2007; Marsh, Yang et al., 2018). Yet, stimuli that are loaded with motivational value such as personal significance and 
emotional valence are known to bias the contents of awareness (e.g., Holeckova, Fischer, Giard, Delpuech, \& Morlet, 2006; Roye et al., 2007; West, Anderson, \& Pratt, 2009). Some authors have proposed that motivational salience is a factor driving attentional selection that increases the priority of a stimulus as if this stimulus was perceptually salient (e.g., Anderson, 2013). Others have suggested that motivationally significant stimuli prompt the formation of long-term representations that modulates attentional processes and leads to qualitatively different and additional subsequent processing of those stimulations (e.g., Roye et al., 2007). In any case, there are automatic biases toward motivationally significant stimuli. Hence, any conclusion about the full automaticity of semantic processing based exclusively on attention-capture effects by particularly meaningful sounds would remain equivocal. In order to elucidate this issue, we assessed whether the semantic content of meaningless—i.e. non-significant—irrelevant sound can also automatically attract attention.

\section{Auditory Deviance}

One obstacle in trying to capture attention with non-significant distractors is that there is nothing inherent in the content of such stimuli per se that endows them with the power to capture attention. Yet, there is evidence that irrelevant stimulations with no inherent motivational value can nonetheless elicit an attentional response when they differ in some way from the prevailing context. Such an aspecific form of attentional capture (cf. Eimer, Nattkemper, Schröger, \& Prinz, 1996) takes place when an infrequent and unexpected acoustical change or deviation follows a repetitive or continuous auditory input. This auditory deviation—or oddball—effect (e.g., Escera et al., 1998; Hughes, Vachon, \& Jones, 2005, 2007; Näätänen, 1990; Parmentier, 2008; Schröger \& Wolff, 1998; Sörqvist, 2010) has nothing to do with the physical properties of the deviant sound per se. The deviant captures attention because it violates a predictable pattern—or regularity—extracted from the incoming sounds (e.g., Bendixen, Roeber, \& Schröger, 2007; 
Vachon, Hughes, \& Jones, 2012; Winkler, Denham, \& Nelken, 2009), regardless of the ongoing cognitive activity (Hughes et al., 2007; Vachon et al., 2017).

A widely accepted assumption is that a (deviant) sound produces attentional capture to the degree that its physical characteristics mismatch the short-lived memory representation of the acoustic invariances automatically extracted from the recent auditory stimulation (Cowan, 1995; Schröger, 1997; Sokolov, 1963). The underlying processes are regarded as preattentive insofar as they are active in the absence of attention. Hence, the attentional response to an acoustical deviant can be taken as evidence for the obligatory processing of the physical properties of the auditory environment. But what of a semantic deviation? By extension, the observation that a rare and unexpected change in the semantic content of a TBI auditory stimulation can capture attention and, in turn, disrupt performance would support the hypothesis that semantic processing is preattentive or automatic. To our knowledge, however, there has been no empirical demonstration of such a deviation effect in the semantic domain.

There is evidence that the semantic content of irrelevant sound can produce interference with task-relevant stimuli in the context of the deviation paradigm. Yet, these semantic distraction effects were found exclusively for acoustically deviant—hence attention-capturingsounds (e.g., Escera, Yago, Corral, Corbera, \& Nuñez, 2003; Parmentier, 2008; Parmentier \& Kefauver, 2015; Parmentier, Turner, \& Perez, 2014; Roye et al., 2007), leading researchers to conclude that the semantic analysis of auditory distractors always follows attention switching toward the deviant stimulus (Escera et al., 2003; Parmentier, 2008; but see Röer, Körner, Buchner, \& Bell, 2017a). More recently, Parmentier and his colleagues (2014) claimed that semantic processing of deviant sounds does not only depend on prior attention capture by acoustical deviation. Contrary to previous studies, the authors found a semantic distraction effect in the absence of an acoustical deviation effect, which led them to conclude that semantic 
processing of the irrelevant sound is not exclusively contingent on acoustic deviance distraction. Yet, their 'semantic deviants' were still physically distinct from the standard sound. Moreover, the semantic content of these deviant words matched the task set for the focal task as it was directly related to the judgment participants had to perform. It is thus possible that the observed semantic distraction effect ensued from the facilitated activation of auditory distractors conveying some task-relevant information. Therefore, this study as well as previous 'deviant' studies do not speak to the question of whether the semantic content of irrelevant sound is processed in a purely obligatory fashion.

\section{The Present Study}

With the general intent of resolving the debate on the automaticity of semantic processing, the purpose of the present study was to establish whether the semantic content of an irrelevant and non-significant sound can be automatically accessed, and if the result of such preattentive processing can interfere with subsequent, this time, deliberate processing. More specifically, we provided the first direct empirical tests of the distractive power of a semantic deviation within an irrelevant auditory stimulation. We capitalized on the established phenomenon whereby a focal visually presented short-term memory task was found to be highly sensitive to disruption by infrequent physical changes in concurrent task-irrelevant auditory stimulation (Hughes et al., 2005, 2007; Lange, 2005; Sörqvist, 2010; Vachon et al., 2012, 2017). Meade and Fernandes (2016) argued that semantic distraction is contingent on the congruency between the semantic properties of a distractor and the task set. To minimize any potential goaldependent capture effects from the semantic content of the irrelevant sound, we used a serial recall task that involved a serial order encoding strategy that tends to promote order processing over item processing (e.g., Beaman \& Jones, 1997; Buschke, 1963; Murdock, 1993). The processes central to supporting serial recall are articulatory-based seriation processes that are not 
semantically-based (e.g., Jones, Macken, \& Nicholls, 2004). Therefore, with serial recall as a focal task, it is thus assumed that attentional control settings should be biased toward the processing of serial information, hence minimizing the activation of language-related-i.e. phonologic, semantic_representations (e.g., Marsh, Hughes, \& Jones, 2008; Marsh, Vachon, \& Jones, 2008). By making the task set incompatible with semantic processing, "rehearsal of order for a serial recall task would lead to a lower likelihood that semantic features of the distractors would capture attention" (Meade \& Fernandes, 2016, p. 802).

In the present set of serial-recall experiments, a visual sequence of seven to eight items (e.g., digits) was presented and participants were requested to recall the items in strict serial order. The presentation of the TBR visual list was accompanied by a TBI auditory sequence composed of acoustically homogenous, 'neutral' (i.e. non-arousing and non-significant) speech tokens drawn from the same category. Previous research has demonstrated that a rare presentation of a single acoustically deviant event within the auditory stream (e.g., a letter spoken in a different voice from that conveying the remaining letters) captures attention as indexed by its disruption of serial recall (e.g., Hughes et al., 2007; Sörqvist, 2010; Vachon et al., 2017). Here, the deviant item, when present, was taken from a different semantic category from the rest of the auditory items (e.g., a digit among letters) so that it diverged from the remaining auditory events exclusively at the semantic level. The use of 'neutral' items was motivated by the fact that such stimuli allow for a change of semantic category that is, at the same time, rather insignificant for the participant. Within this experimental context, the disruption of serial recall by such a categorical change alone-i.e. without any acoustical deviation-would indicate that non-significant extraneous auditory information is endowed with the power to elicit an involuntary attentional response, suggesting in turn that semantic processing takes place automatically. More specifically, the presence of a categorical deviation effect would reveal for 
the first time that it is the semantic relationship between consecutive words within the unattended speech that is automatically processed.

In the present study, we attempted to diagnose the automaticity of semantic processing by examining some of its component features, as defined by Moors and De Houwer (2006, 2007). This investigation was undertaken in two distinct steps. The first step was designed to determine whether semantic processing is goal-independent. Indeed, the first three experiments looked at whether the semantic content of auditory distractors can disrupt the execution of a completely unrelated visual focal task. In the second part of this article, we addressed more specifically whether semantic processing is uncontrollable by testing across four experiments the susceptibility of the semantic deviation effect to top-down control. Given that the semantic material was always task-irrelevant and presented in a TBI channel, the whole set of experiments speak to whether semantic processing can take place unintentionally. Table 2 provides an overview of the experimental work accomplished in every experiment of the current study.

\section{Experiment 1}

Experiment 1 aimed to establish whether an unexpected change in the semantic content of an auditory stimulation could capture attention even when the auditory context is irrelevant to an ongoing visual task. In the context of the irrelevant sound paradigm, participants were asked to recall the serial order of either visually-presented digits or letters while being simultaneously presented with a TBI auditory stream. Overlearned stimuli such as alphanumerical characters were used as TBR items to minimize errors due to the identity of the item being forgotten (e.g., Beaman \& Jones, 1997). Hence, most errors will be ones of order. TBI auditory sequences were composed of items that were emotionally neutral, had no particular significance to participants, 


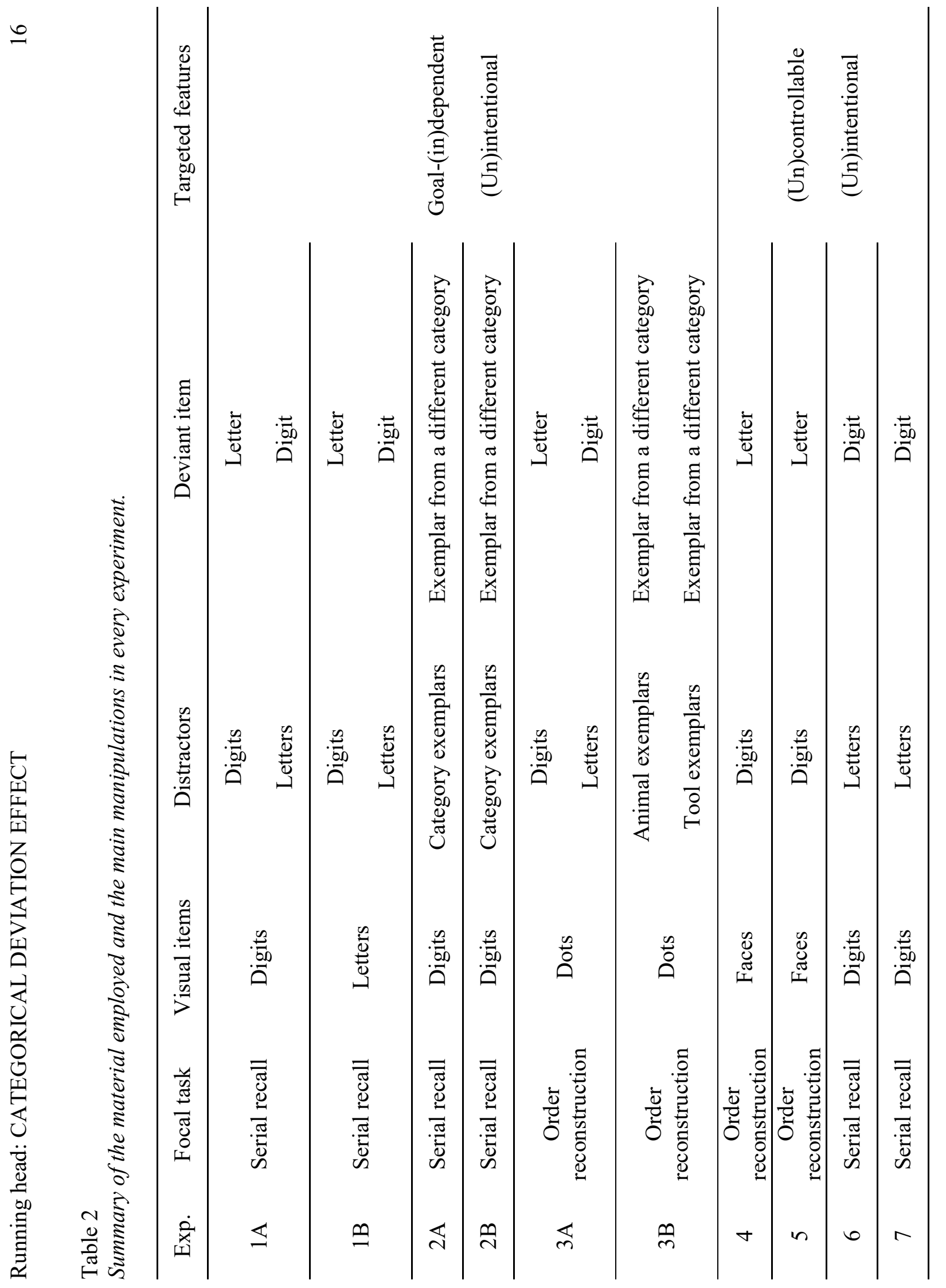


and were irrelevant to the serial recall task. Half of TBI sequences mainly consisted of spoken digits, whereas the other half mainly consisted of spoken letters. Therefore, auditory sequences were composed of items always drawn from the same category (letters or digits). Yet, on some infrequent trials, an unexpected change of category—or categorical deviation —was inserted within the TBI auditory stream. This means that when TBI sounds were digits, the deviant item was a letter and vice versa. As for the acoustical deviation effect, the distractive power of this categorical deviation was assessed by comparing serial recall performance between control and deviant trials. If the categorical deviant is endowed with the power to capture attention, performance should be poorer in deviant trials than in control trials. The experiment was comprised of two experimental blocks that differed with regard to the semantic category of the auditory items (digits vs. letters). In Experiment 1A, participants had to recall visual lists of digits whereas in Experiment 1B, TBR lists were composed of letters. Within such a design, TBR and TBI items came from the same category in one block of trials (e.g., visual digits and auditory digits) and from different categories in the other block (e.g., visual digits and auditory letters). Such a manipulation was made to determine whether TBR and TBI material had to be semantically related in order for a categorical deviation effect to take place.

Given that in the Introduction, we stressed the importance of minimizing the relationship between the relevant and irrelevant stimuli when assessing the automaticity of semantic processing, our choice of design in this experiment may appear surprising. Indeed, not only the same items composed both the visual and auditory sequences in one block of trials, but also the auditory deviant item was drawn from the same category as the visual memoranda in the other block. We exploited this latter feature of our design to characterize the nature of the deviant disruption. Indeed, using a deviant item that can be retrieved as a potential candidate for output allowed for the assessment of intrusions of that key distractor. The underlying notion is that if 
attention is allocated to the deviant, its identity should be processed (Parmentier, 2008), promoting the inappropriate recall of that item (cf. identity intrusion technique; Theeuwes \& Burger, 1998). Given that the TBR sequences were composed of eight visual items drawn from a 9-item stimulus set, one item from the set was always excluded. For instance, if the visual sequence was composed of the digits 1 to 8 , the digit 9 was omitted. The incorrect recall of this 'missing' item was thus considered as an intrusion. Typically, extra-list intrusions tend to be quite rare in the serial recall setting (Surprenant, Neath, \& Brown, 2006). In deviant trials where TBR and TBI items were drawn from different categories, the deviant item was automatically part of the same category as the TBR items. In such cases, we made sure that the identity of the missing item always corresponded to that of the deviant item. In the previous example, if the digit ' 9 ' was excluded from the visual list on a deviant trial, it was presented as the deviant digit among spoken letters. We assumed that if the categorical deviant involuntarily attracts attention, this irrelevant item is likely to receive further processing of its identity. Such enhanced processing should in turn promote the incorrect recall—or intrusion—of that (deviant) auditory stimulus as a visual item. Therefore, if the occurrence of a categorical deviation truly captures attention, it should not only translate into poorer serial recall but also into increased intrusions of the missing item.

\section{Method}

Participants. Thirty adults (12 women; mean age: 24.3 years) took part in Experiment 1A then another 20 adults (14 women; mean age: 30.1 years) participated in Experiment 1B. For all the experiments of this study, volunteers were recruited on the campus of Université Laval and received a small honorarium for their participation. They were first asked to complete a written consent form and a questionnaire about their age, gender, education level, and history of vision or hearing impairments. They all reported normal hearing and normal or corrected-to- 
normal vision. The research reported in the present article received approval from the ethics committee of Université Laval.

Materials. The experiment was controlled by a PC computer using E-Prime 2.0 Professional (Psychology Software Tools). To-be-remembered visual stimuli were presented on a computer screen located at approximately $60 \mathrm{~cm}$ from the participant while to-be-ignored auditory stimuli were presented binaurally through headphones at approximately $65 \mathrm{~dB}(\mathrm{~A})$.

TBR visual stimuli. All visual sequences were eight items in length. In Experiment 1A, TBR items were taken without replacement from the digit set $1-9$ and arranged in a quasirandom order, with the constraint that successive digits were not adjacent integers. In Experiment 1B, the items were taken without replacement from the letter set B, F, H, K, M, Q, $\mathrm{R}, \mathrm{X}$, and $\mathrm{Z}$, and arranged in a random order. Each item was approximately $2.39^{\circ}$ in height and presented sequentially in a black Times New Roman font at the center of a white background. Each item was presented for $250 \mathrm{~ms}$ and the interstimulus interval (offset to onset) was $500 \mathrm{~ms}$.

TBI auditory stimuli. For the irrelevant auditory sequences, two sets of the French spoken items were recorded in a male voice: the digit set $1-9$ and the letter set B, F, H, K, M, Q, $\mathrm{R}, \mathrm{X}$, and Z. Note that these letters are phonologically dissimilar in French. Each item was spoken at an approximately even pitch and edited using SoundForge (Sony) to last $250 \mathrm{~ms}$. The interstimulus interval was $500 \mathrm{~ms}$ so that the visual and auditory sequences were synchronized. Using these sounds, we generated four types of TBI sequence:

1. Letters: This type of sequence was composed of eight letters randomly selected from the set of nine spoken letters. Letters were presented in a random order.

2. Letters + deviant digit: A sequence of this type was identical to the Letters sequencetype except that the fifth letter, the onset of which coincided with the onset of the fifth TBR item, 
was replaced by one of the nine spoken digits. In Experiment 1A, the deviant spoken digit corresponded to the digit that was absent from the TBR sequence.

3. Digits: This type of sequence was composed of eight digits randomly selected from the set of nine spoken digits. The items were presented in a quasi-random order, with the constraint that successive digits were not adjacent integers.

4. Digits + deviant letter: A sequence of this type was identical to the Digits sequencetype except that the fifth digit, the onset of which coincided with the onset of the fifth TBR item, was replaced by one of the nine spoken letters. In Experiment 1B, the deviant spoken letter corresponded to the letter that was absent from the TBR sequence.

One could argue that spoken digits are more acoustically complex than spoken letters since they sound like spoken words. On this logic, a change of category may have indirectly induced a change in acoustical complexity. If so, any disruption found in deviant trials could in fact reflect, at least in part, an acoustical distraction effect. To test this possibility, we contrasted key acoustical and phonological properties of the two sets of spoken stimuli. The analysis focused more specifically on acoustic characteristics such as stimulus duration, loudness and spectral composition and on phonological features such as the number of syllables and phonemes as well as the phonological uniqueness point. Methodological details and results are presented in Appendix A. The analysis revealed that there was no systematic discrepancy at either acoustical or phonological level between the spoken letters and the spoken digits employed in the present experiment that could account for any disruption produced by the introduction of a categorical deviant item within a sequence of spoken alphanumerical stimuli.

Design and procedure. In Experiment 1A, participants performed serial recall of visual digits whereas they carried out serial recall of visual letters in Experiment 1B. This yielded a $2 \times$ $2 \times 2$ mixed design, which comprised two within-subject factors: Type of sound (letters or digits) 
and Deviation (whether or not the auditory sequence contained a categorical deviation), and the between-subjects factor Type of TBR material (Exp. 1A: digits and Exp. 1B: letters). In each experiment, 80 serial recall trials were divided into two blocks: The 'letter' block consisted of 40 trials with TBI auditory sequences composed of spoken letters and the 'digit' block was comprised of 40 trials with TBI spoken digits. The order of the two blocks was counterbalanced across participants. Each block contained 32 standard—or non-deviant—trials and 8 deviant trials. In the 'letter' block, the deviant trials were Trials 5, 8, 15, 21, 26, 30, 36, and 39 whereas in the 'digit' block, the deviant trials were Trials $4,10,14,19,25,28,35$, and 40.

Participants were tested individually in a sound-attenuated booth. They read standard instructions that informed them of what the serial recall task involved. They were also told that sound would be presented over the headphones but that it was irrelevant to their task and that they were therefore to ignore it. They were not told about the presence of deviant events within the sound. Participants were informed that the trials would be presented at a pre-set pace. Fifty ms following the offset of both TBR and TBI sequences, the screen flashed from white to black for $150 \mathrm{~ms}$, which signaled to the participants that they should begin to write out the TBR list in an answer booklet containing 82 rows of eight blank squares ( 2 for the practice trials, and 80 for the experimental trials). From the offset of the screen flashing, there were $15 \mathrm{~s}$ before the presentation of the first item of the next TBR list. Thirteen seconds into the $15 \mathrm{~s}$ of writing time, a 500-ms tone was presented over the headphones to signal to the participant that the presentation of the first item of the next sequence was imminent. There were two standard practice trials before the first block (one 'TBI letters' trial and one 'TBI digits' trial). Including an optional 5-min break between blocks, the experiment lasted approximately $40 \mathrm{~min}$.

Analyses. Here, and applicable elsewhere in this study, all data were analyzed using the analysis of variance (ANOVA) technique with an alpha level of .05. For each main effect and 
interaction effect, we reported the classical $F$ and $p$ values along with an estimate of the effect size $\left(\eta_{\mathrm{p}}^{2}\right)$ as well as the probability that the data favor the null hypothesis over the alternative hypothesis $\left[p_{\mathrm{BIC}}\left(\mathrm{H}_{0} \mid \mathrm{D}\right)\right]$ as computed by a Bayes factor analysis using Masson's (2011) method.

Two dependent variables were analyzed: recall performance and intrusion errors. With regard to performance, the raw data were scored according to the strict serial recall criterion: To be recorded as correct, an item had to be recalled in its original presentation position. An intrusion is committed when an item that had not been presented in the just-presented sequence is recalled. In the present experiments, an intrusion corresponded to the erroneous recall of the 'missing' item, i.e. the only item from the original set of nine visual items that was absent from the TBR list. When drawn from the same category as the TBR items, the deviant identity always corresponded to that of the 'missing' item (i.e. in 'Letters + deviant digit' trials of Experiment $1 \mathrm{~A}$ and in 'Digits + deviant letter' trials of Experiment 1B). This manipulation allowed verification of whether the categorical deviant tended to be processed and then falsely recalled.

\section{Results}

Serial recall. Figure 1 presents the percentage of digits correctly reported in the four conditions of Experiment 1A (left panel) and Experiment 1B (right panel). Here, and applicable elsewhere in this study, error bars represent $95 \%$ within-subject confidence intervals computed using Cousineau's (2005) method with Morey's (2008) correction. Serial recall performance appeared to be lower on deviant trials regardless of the type of TBI sound and the type of TBR material. These data were submitted to a $2 \times 2 \times 2$ mixed ANOVA with Type of TBR material (Exp. 1A: digits, and Exp. 1B: letters) as the between-subjects factor and Type of sound (letters or digits) and Deviation (with or without deviant) as within-subject factors. The ANOVA revealed a significant main effect of Deviation, $F(1,48)=34.07, p<.001, \eta_{\mathrm{p}}^{2}=.415, p_{\mathrm{BIC}}\left(\mathrm{H}_{0} \mid \mathrm{D}\right)$ 
$<.001$, confirming that serial recall was poorer in the presence of a categorical deviation. The main effects of Type of Sound, $F(1,48)=1.13, p=.293, \eta_{\mathrm{p}}^{2}=.023, p_{\mathrm{BIC}}\left(\mathrm{H}_{0} \mid \mathrm{D}\right)=.798$, and Type of TBR material were not significant, $F(1,48)=2.69, p=.107, \eta_{\mathrm{p}}^{2}=.053, p_{\mathrm{BIC}}\left(\mathrm{H}_{0} \mid \mathrm{D}\right)=.644$.

None of the interaction effects were significant $\left(F \mathrm{~s}<1.43, p \mathrm{~s}>.238, \eta_{\mathrm{p}}^{2} \mathrm{~s}<.03, p_{\mathrm{BIC}}\left(\mathrm{H}_{0} \mid \mathrm{D}\right) \mathrm{s}>\right.$ .774), suggesting that the deviation effect was not influenced by the type of stimuli presented in the visual and auditory channels.
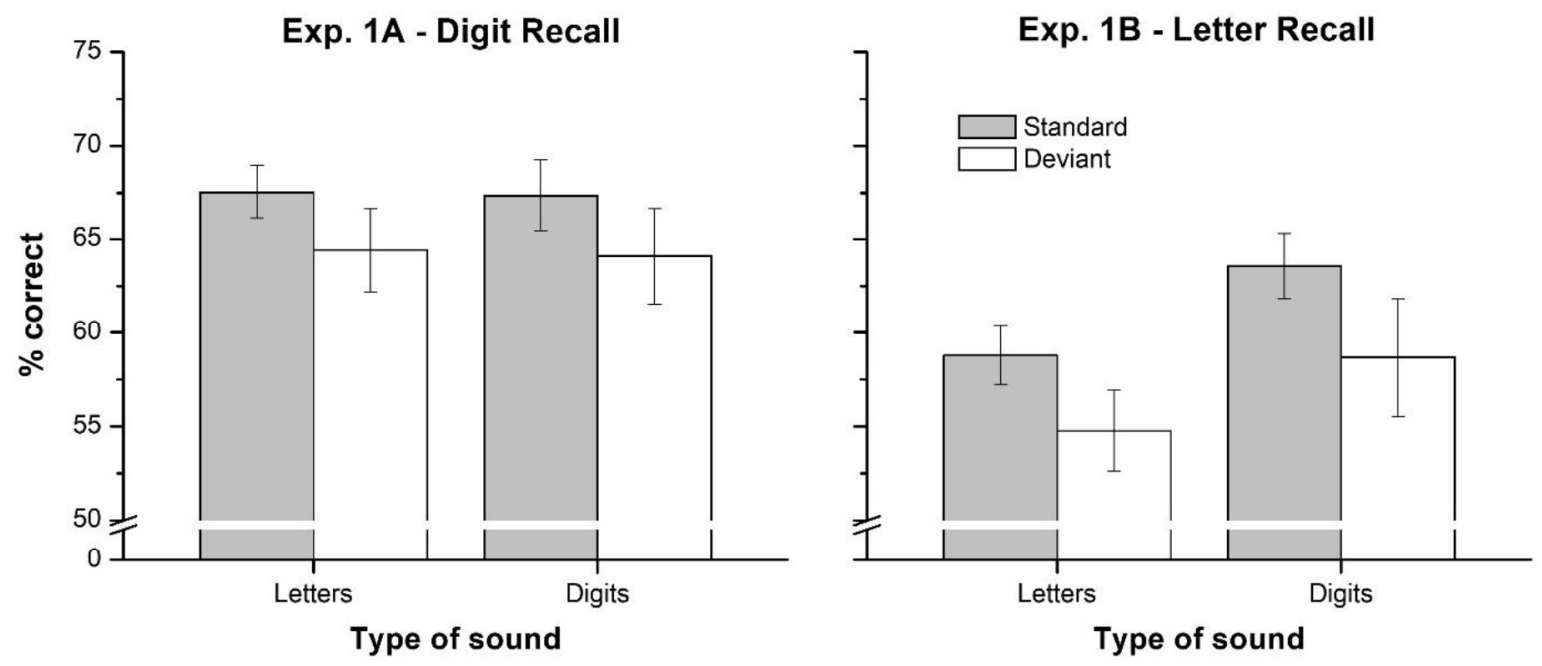

Figure 1. Mean percentage of items correctly recalled in the four conditions of Experiments $1 \mathrm{~A}$ (digit recall) and 1B (letter recall). Error bars represent 95\% within-subject confidence intervals.

Intrusions. Figure 2 shows the percentage of 'missing-item' intrusions for standard and deviant trials of both Experiments $1 \mathrm{~A}$ and 1B. The percentage of intrusions was submitted to a 2 $\times 2$ mixed ANOVA with Type of TBR material as the between-subjects factor and Missing item (absent vs. presented as the deviant) as the within-subject factor. The analysis revealed a significant main effect of Missing item, $F(1,48)=14.32, p<.001, \eta_{\mathrm{p}}^{2}=.230, p_{\mathrm{BIC}}\left(\mathrm{H}_{0} \mid \mathrm{D}\right)=.010$, indicating that intrusions of the missing item were more likely when it was presented as a deviant spoken item. There was no main effect of Type of TBR material, $F(1,48)=0.15, p=.703, \eta_{\mathrm{p}}^{2}=$ 
$.003, p_{\mathrm{BIC}}\left(\mathrm{H}_{0} \mid \mathrm{D}\right)=.867$. More importantly, the interaction was not significant, $F(1,48)=1.39, p$ $=.244, \eta_{\mathrm{p}}^{2}=.028, p_{\mathrm{BIC}}\left(\mathrm{H}_{0} \mid \mathrm{D}\right)=.777$, suggesting that the higher rate of intrusions found for when the missing item corresponded to the deviant identity was found for both types of TBR material.

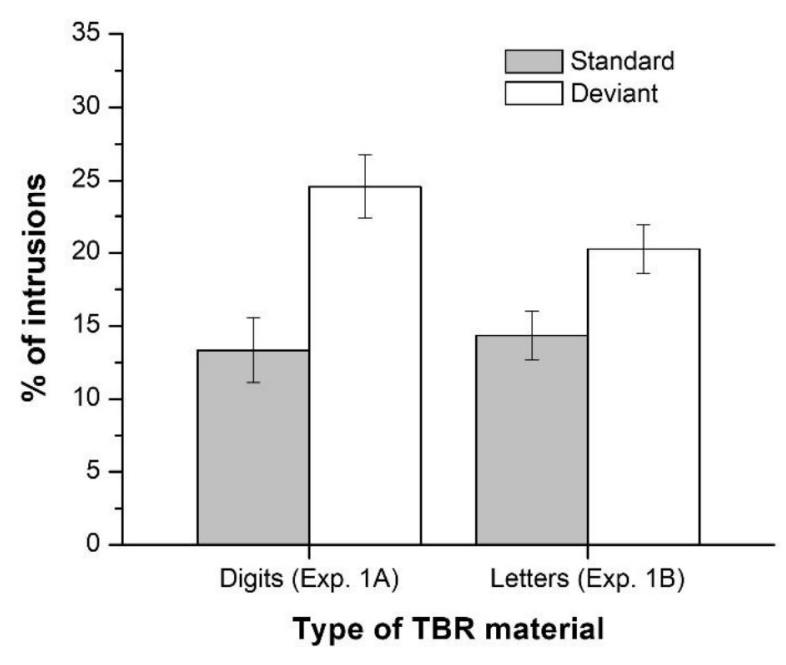

Figure 2. Mean percentage of intrusions of the missing item when absent from the trial (standard) and when presented as the auditory deviant (deviant) in Experiments 1A (digit recall) and 1B (letter recall). Error bars represent $95 \%$ within-subject confidence intervals.

\section{Discussion}

The results showed for the first time that an unexpected change of category within the TBI auditory sequence disrupted visual serial recall. This occurred regardless of whether the TBR and TBI stimuli belonged to the same semantic category or not. Coupled with the more frequent intrusions of the 'missing' item when presented as an auditory deviant than when absent from the auditory stimulus presentation, such disruption strongly suggests that an orienting response was triggered by the deviant. While intrusions in control trials probably ensued from a guessing strategy since the missing item was a plausible candidate of the TBR list, the more frequent intrusions of the deviant item are likely to reflect a failure of the source-monitoring process prompted by attention capture (see Marsh, Hughes, \& Jones, 2008). Indeed, the 
involuntary reallocation of attention from the visual stimulus presentation to the auditory deviant might have rendered participants more prone to confusing the source of activation of the deviant's representation, attributing it to the visual TBR list instead of the irrelevant auditory sequence (see Johnson, Hashtroudi, \& Lindsay, 1993; Marsh, Hughes, \& Jones, 2008). Not only do these findings indicate that a categorical deviation is, like acoustical deviations, endowed with the power to capture attention, they also provide evidence that the auditory sequence was processed to the semantic level despite its content having no personal significance or arousalinducing properties and being irrelevant to the focal task.

Importantly, this distraction effect cannot be ascribed to acoustical factors. Indeed, we provided a matching of deviants and standards for several acoustic and phonological variables (see Appendix A) that ruled out an acoustical origin view of the deviation effect highlighted in the present experiment. We are thus confident that the categorical deviation effect ensued from the detection of an unexpected variation in the semantic content of the irrelevant auditory sequence.

The question here is whether the analysis of the semantic content of the irrelevant sound took place goal-independently or not. That a categorical deviation effect was found under serial recall, a task minimizing the amount of attention being paid to the semantic properties of the TBR items (Meade \& Fernandes, 2016), suggests that attentional responses to categorical deviants were triggered in a non-contingent fashion, likewise for orienting responses to acoustical deviations (see Vachon et al., 2017). Consistent with this hypothesis is the fact that categorical deviants disrupted recall even when drawn from a different semantic category (e.g., digits) than the TBR material (e.g., letters). If attentional capture by a categorical deviation was indeed elicited independently of the participant's task set, it could then be argued that the results of Experiment 1 are consistent with the view that semantic processing is goal-independent. 
Even though the attentional control settings for serial recall are incompatible with the (semantic) properties of the deviant distractor causing interference (cf. Meade \& Fernandes, 2016), it is nevertheless possible that the categorical deviation effect highlighted in the present experiment was contingent on the close relationship between the TBR and TBI material. Such contingency would imply the involvement of top-down mechanisms in the semantic activation of the irrelevant auditory sequence, therefore arguing against the automaticity of semantic processing. One way by which the extraction of the content of the irrelevant sound may have been promoted is through the application of a proactive control strategy to perform the serial recall task. Braver (2012) proposed that cognitive control operates via two different operating modes: a proactive (or preempting; see Ansorge \& Horstmann, 2007; Kiefer, 2007) control mode, whereby task goals are actively maintained to optimally prepare the cognitive system to respond most effectively to subsequent events, and a reactive control mode, under which goal representations are only activated when required. In the context of the irrelevant sound paradigm, in which participants must focus on visual TBR stimuli while ignoring auditory distractors, the adoption of a proactive control mode would be particularly advantageous given that this mode of control tends to optimize the processing of task-relevant information over competing sources of information. Indeed, "proactive control relies upon the anticipation and prevention of interference before it occurs" (Braver, 2012, p. 106). Under proactive control, the appropriate task set can be activated and used to predict the identity of the incoming stimuli, which implies that the semantic category of the TBR items should have been represented in that task set. Given that in the present experiment a (deviant) category change in the auditory sequence always involved the task-set activated semantic category, a proactive control mode could have left participants open to inadvertently processing this irrelevant category switch. 


\section{Experiment 2}

In the previous experiment, we purposely used TBR and TBI material belonging to the same class of stimuli in order to examine potential intrusions of the identity of the categorical deviant into serial recall. Yet, the use of spoken alphanumerical stimuli may be not optimal for two main reasons. First, because of the close relationship between task-relevant and taskirrelevant information, it is impossible to determine whether the disruption of serial recall by a categorical deviation found in Experiment 1 reflects the action of contingent or non-contingent distraction mechanisms. Second, although a change from one stimulus category (e.g., digits) to another (e.g., letters) constitutes a post-categorical deviation—and gave rise to disruption—such auditory stimuli were quite impoverished in terms of semanticity. One could therefore argue that the locus of the categorical deviation effect observed so far is located prior to the semantic level, making any conclusion about the automaticity of semantic processing equivocal.

To address these issues, we used in Experiment 2 homogenous exemplars from semantically rich taxonomic categories as auditory distractors. In this experiment, each TBI auditory sequence was composed of spoken words taken from the same semantic category (e.g., Fruits: apple, pear, grape, orange...). On deviant trials, one auditory distractor was replaced by an exemplar of a different category (e.g., Tools: hammer). In this context, the detection of a categorical change — that is responsible for causing disruption—would require the irrelevant sound to be analyzed at the semantic level. Therefore, the observation of a categorical deviation effect using category exemplars would provide strong evidence that the phenomenon ensues from the (automatic) semantic processing of the TBI sound. Finally, using spoken category exemplars as TBI items in the context of the serial recall of alphanumerical visual stimuli helped minimize the relationship between the memoranda and the distracting information. In such a context, the categorical deviation effect should vanish if it reflects contingent attentional capture. 
Bell, Röer, Marsh, Storch, and Buchner (2017) have previously reported a deviation effect using TBI sequences of spoken words. They found significant disruption of serial recall when a novel word (e.g., dog) was embedded in an irrelevant auditory sequence composed of the repetition of the same one-syllable word (e.g., "black, black, black, black..."). While the insertion of a novel word in that study could be considered as categorical deviation, it clearly also induced an acoustical deviation. Moreover, not all of the words were taken from semantic categories. It is therefore impossible to determine whether a change in semantic content was responsible for the deviation effect reported by Bell and colleagues.

In the previous experiment, only two types of auditory distractor were employed (i.e. digits and letters) so that the category of the TBI items remained the same throughout an entire block of trials. The use of several different categories entails the possibility of changing from trial to trial the category from which the spoken distractors are drawn. Such a possibility is interesting in the context of the present study because it allows to test another prediction of the automatic semantic activation view. If the semantic content of the irrelevant auditory sequences is extracted automatically, then the semantic context should be established after only a few exemplars of the same category have been encountered. From an automatic semantic activation standpoint, therefore, the disruptive power of a categorical deviation should not be influenced by whether the category from which the distractors are drawn is kept constant or changes on every trial. Experiment 2 was designed to test this prediction. In Experiment 2A, a single category of irrelevant spoken words was selected randomly for each participant and used throughout the entire experiment, as in previous experiments. For instance, one participant may have to ignore auditory sequences composed of animal exemplars (e.g., cat, dog, mouse, etc.) on every trial. In Experiment $2 \mathrm{~B}$, the category from which the distractors were taken changed randomly on a trialto-trial basis. If the categorical deviation effect is underpinned by the automatic access to the 
meaning of the irrelevant sound, disruption of similar magnitude is expected in both conditions. To simplify the design, serial recall was this time restricted to lists of visual digits.

Drawing non-deviant distractors from the same category on every trial can also be problematic because the same small pool of distractors (from a single category) was recycled from trial to trial. In such a case, a categorical deviant would differ from the other distractors not only in terms of its semanticity but also of its rarity. One could therefore argue that the disruption found in previous experiments reflects distraction in response to (acoustical) novelty (cf. Escera et al., 1998) rather than to categorical deviance. Employing multiple categories allows to test this novelty account of the categorical deviation effect. Indeed, with multiple categories, it becomes possible for a category exemplar presented as a deviant item in a deviant trial to also be presented as a non-deviant distractor in a control trial. In such a case, a deviant item would not be novel anymore. Therefore, in Experiment 2B, we ensured that each categorical deviant had been encountered as a non-deviant distractor beforehand so that this stimulus would lose its novelty. If the disruptive impact of a categorical deviant is mainly driven by its novelty, then the categorical deviation effect should be larger for repeated categories (Experiment 2A) than for multiple categories (Experiment 2B).

\section{Method}

The method was identical to that employed in Experiment 1A (digit serial recall), except as noted below.

Participants. Twenty adults (11 women; mean age: 22.4 years) took part in Experiment 2A while another 20 adults (14 women; mean age: 23.6 years) participated in Experiment 2B. None of them had taken part in previous experiments.

Materials. TBI auditory stimuli consisted of 16 category exemplars drawn from 36 different semantic categories (e.g., flowers, weather phenomena, musical instruments, etc.). All 
recorded words were French spoken in a male voice. All sound files lasted $750 \mathrm{~ms}$, but depending on their length, TBI words could be presented for less than $750 \mathrm{~ms}$. On each trial, the auditory sequence was composed of eight words taken without replacement from the set of 16 words pertaining to a single semantic category (e.g., for the semantic category "animals": 'cat', 'mouse', 'goat', 'fox', 'wolf', 'horse', 'rabbit', and 'pig'). On deviant trials, a word drawn from a different semantic category was inserted in $6^{\text {th }}$ position of the sequence (e.g., 'hamster', 'dog', 'deer', 'pig', 'moose', 'knee', 'bear', and 'cow').

In Experiment 2A, all auditory sequences were comprised of category exemplars drawn from only one of four possible semantic categories: animals, tools, fruits, or occupations. The selected semantic category was counterbalanced across participants. When present, the deviant word was taken without replacement from any of the remaining 35 semantic categories. In Experiment 2B, the semantic category of TBI sequences changed from trial to trial in a quasirandom fashion so that a semantic category was never used in consecutive trials. Each of the 36 semantic categories was used two or three times throughout the experiment. With the exception of the first trial, each semantic deviant had been presented first as a non-deviant distractor. Thus, the non-deviant and deviant exemplars were very rarely novel stimuli when they were presented.

As in Experiment 1, we performed an analysis of the acoustical and phonological properties of the spoken stimuli used in the present experiment in order to rule out the possibility that distraction effects ensued from disparities in low-level auditory features between deviant and standard exemplars. Such an analysis was carried out separately for the spoken stimuli used in Experiment 2A (see Appendix B) and those employed in Experiment 2B (see Appendix C). The results revealed that, overall, there were no systematic acoustical and/or phonological discrepancies between deviant and standard exemplars of both Experiments 2A and 2B, with one exception. Indeed, we observed that the occupation (standard) exemplars used in Experiment 2A 
seemed more phonologically complex than the deviant exemplars. Under such conditions, any deviation effect could therefore be attributable, at least in part, to acoustic factors. Accordingly, we analyze serial recall performance with and without this set of stimuli to make sure the observed phonological disparity cannot fully account for the pattern of results obtained.

Procedure. Participants performed two blocks of 45 serial-recall trials, their order being counterbalanced across participants. Each block contained 39 standard—or non-deviant—-trials and 6 deviant trials. In one block, the deviant trials were Trials 5, 14, 18, 25, 35, and 43 whereas in the other block, the deviant trials were Trials 2, 7, 16, 20, 28, and 39.

\section{Results}

Figure 3 presents the percentage of digits correctly reported in standard and deviant trials of Experiments 2A and 2B. Serial recall performance appeared to be lower on deviant trials in both experiments. These data were submitted to a $2 \times 2$ mixed ANOVA with TBI category (Exp. 2A: single, and Exp. 2B: multiple) as the between-subjects factor and Deviation (with or without deviant) as the within-subject factor. The ANOVA revealed a significant main effect of Deviation, $F(1,38)=30.38 p<.001, \eta_{\mathrm{p}}^{2}=.444, p_{\mathrm{BIC}}\left(\mathrm{H}_{0} \mid \mathrm{D}\right)<.001$, confirming that serial recall was poorer in the presence of a categorical deviation. Neither the main effect of TBI category, $F(1,38)=0.07 p=.790, \eta_{\mathrm{p}}^{2}=.002, p_{\mathrm{BIC}}\left(\mathrm{H}_{0} \mid \mathrm{D}\right)=.859$, nor the interaction were significant, $F(1$, $38)=1.08 p=.305, \eta_{\mathrm{p}}^{2}=.028, p_{\mathrm{BIC}}\left(\mathrm{H}_{0} \mid \mathrm{D}\right)=.795$, suggesting that the deviation effect took place regardless of whether the semantic category from which TBI sound were drawn changed from trial to trial or remained the same throughout the experiment.

Given the phonological mismatch found between the occupation exemplars and the deviant exemplars of Experiment 2A, data from the five participants who were presented with spoken exemplars taken from the occupation category were removed from the ANOVA to verify 
that the disruption obtained was not attributable to this phonological discrepancy. The pattern of results remained unchanged: the main effect of Deviation was still significant, $F(1,33)=24.37 p$ $<.001, \eta_{\mathrm{p}}^{2}=.425, p_{\mathrm{BIC}}\left(\mathrm{H}_{0} \mid \mathrm{D}\right)<.001$, while the main effect of TBI category and the interaction remained non-significant $\left(F_{\mathrm{S}}<1\right)$. When analyzed in isolation, the deviation effect obtained in Experiment 2A was significant regardless of whether participants from the occupation condition were included, $t(19)=3.18, p=.005, d_{z}=0.71$, or not, $t(14)=2.56, p=.023, d_{z}=0.66$. It is therefore clear that phonological disparities cannot account for the categorical deviation effect obtained in Experiment 2A.

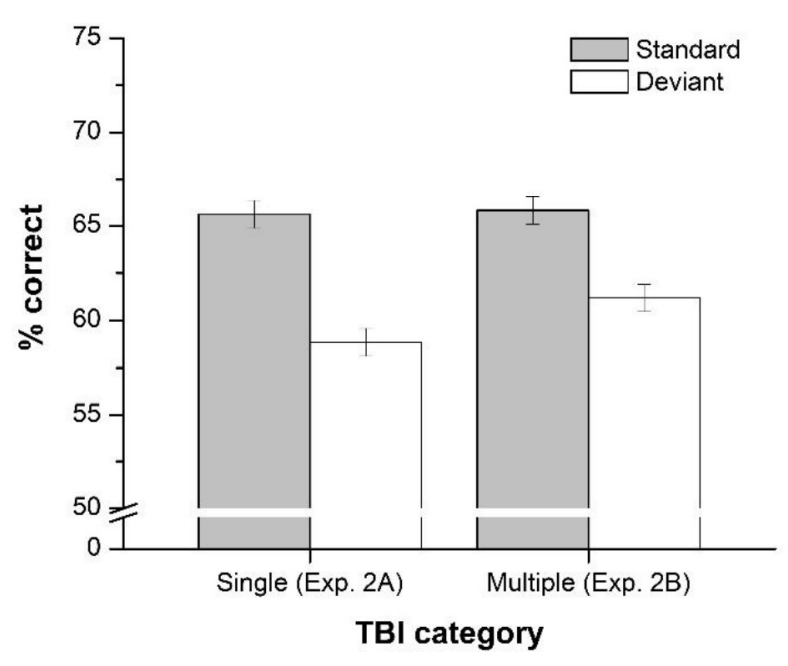

Figure 3. Mean percentage of items correctly recalled in standard and deviant trials of Experiments 2A (single to-be-ignored [TBI] category) and 2B (multiple TBI categories). Error bars represent $95 \%$ within-subject confidence intervals.

\section{Discussion}

Experiment 2 replicated the categorical deviation effect found in Experiment 1 by this time using TBI exemplars taken from semantically rich taxonomic categories. Therefore, Experiment 2 conceptually replicates the findings of the previous experiment and furthermore removes any uncertainty that the categorical deviation effects reported to this point were 
produced due to pre-semantic analysis of the irrelevant sound. Moreover, a comparison of the physical properties of the deviant and standard exemplars employed as auditory distractors demonstrated that the disruption reported in Experiments 2A and 2B cannot be explained in terms of acoustical/phonological factors. Such findings add further weight to the notion that the categorical deviation effect observed in the foregoing experiments is attributable to processing at the semantic level, consistent with the notion of automatic semantic processing. The fact that the distractive power of the deviant items was similar whether they differed from non-deviants in terms of both semanticity and novelty (Experiment 2A) or exclusively in terms of semanticity (Experiment 2B) rules out the novelty view of the categorical deviation effect.

In addition, results from Experiment 2 revealed that the disruptive power of the categorical deviant was not influenced by the establishment of a new semantic context within the TBI stream on every trial. In fact, Experiment 2B showed that the presentation of as few as five exemplars of the same category was sufficient to set up a semantic context strong enough for the insertion of an exemplar from another category to be detected and, consequently, to become disruptive. This finding that a semantic context is quickly established following the presentation of a few distractors is consistent with the view that the content of the irrelevant sound is analyzed at the semantic level in an automatic fashion.

The replication of the categorical deviation effect obtained in Experiment 1 with TBI sounds - i.e. category exemplars — that were not related to the TBR items-i.e. alphanumerical characters - provides evidence that attentional capture by categorical deviation is non-contingent on the activated task set, hence suggesting that the semantic content of the irrelevant sound was processed in a goal-independent fashion. Yet, since having to recall alphanumerical stimuli may have promoted the use of a verbal encoding strategy, one cannot preclude the hypothesis that the categorical deviation effect found in the previous experiments may be dependent upon the 
activation of verbal codes in the focal task (see also Röer et al., 2017b). Although in our view this is unlikely, we examined the categorical deviation effect in again Experiment 3, this time using TBR material minimizing the possibility that participants could have recourse to a verbal encoding strategy.

\section{Experiment 3}

This experiment was designed to further minimize the relationship between TBR and TBI material in the context of the irrelevant sound paradigm. Here, we asked participants to reconstruct the order of presentation of a set of spatially distributed dots (see, e.g., Guérard \& Tremblay, 2008; Jones, Farrand, Stuart, \& Morris, 1995; Vachon et al., 2017). In each trial, a set of eight TBR dots was presented one at a time using a random permutation of the same eight fixed locations illustrated in Figure 4. Following the presentation of the TBR list, all dots reappeared simultaneously on the screen and participants were required to click on them in their order of presentation. The application of arbitrary verbal labels for each position (e.g., "top-left", "bottom-right") is unlikely because the positions of the dots on the screen were not always visible and were such that the locations were difficult to verbalize (Jones et al., 1995). Besides, this 'dot task' is known to preclude the deliberate use of verbal codes to remember spatial positions (see Jones et al., 1995, for a discussion). This visuospatial version of the serial recall task is, as its verbal counterpart, susceptible to auditory deviance: the reconstruction of the order of the dots can be disrupted by acoustical deviants (Marsh, Vachon, \& Sörqvist, 2017; Morey \& Miron, 2016; Vachon et al., 2017). The observation of a categorical deviation effect in the present setting would constitute convincing evidence that the semantic processing of the TBI stimuli is not conditional on the activation of verbal codes in the focal task.

We examined the categorical deviation effect in the context of the dot task using the types of auditory material employed in the previous experiments. In Experiment 3A, irrelevant 
auditory sequences were composed of alphanumerical stimuli (i.e. digits and letters), whereas they were composed of category exemplars in Experiment 3B. In order to keep some equivalence across the two versions of the current experiment, we chose to limit the number of categories used in Experiment 3B to two. Hence, participants in Experiment 3B were presented with auditory sequences containing either animal or tool exemplars.

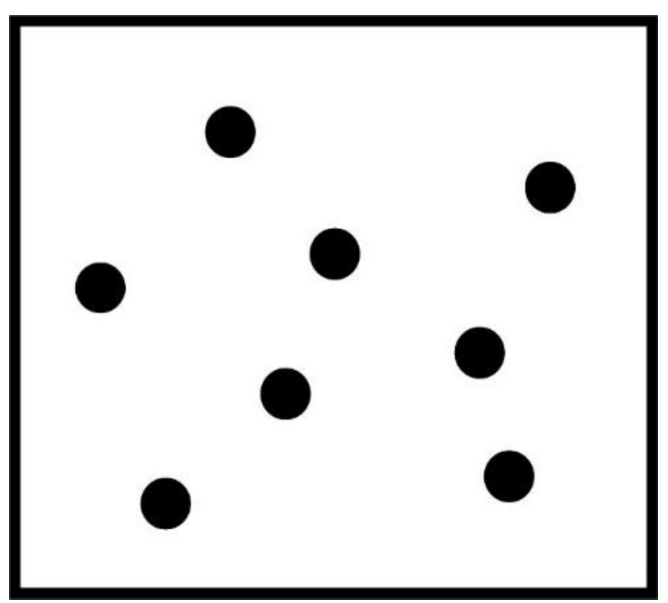

Figure 4. Spatial configuration of the to-be-remembered stimuli used in Experiment 3.

\section{Method}

Participants. Thirty volunteers (17 women; mean age: 22.8 years) took part in Experiment 3A while another 30 volunteers ( 20 women; mean age: 24.2 years) participated in Experiment 3B. These new participants had the same characteristics as those who took part in previous experiments.

Materials. In both Experiments 3A and 3B, TBR sequences were now composed of eight black dots of approximately $2.00^{\circ}$ in diameter, displayed at different spatial locations within a white $21.8^{\circ} \times 21.8^{\circ}$ window (see Figure 4 ). Each of these sequences was constructed using a quasi-random permutation of the same fixed eight dot locations, with the constraint that sequences could not share more than four dots having an identical combination of spatial location and serial position. Each dot was presented at each serial position between 8 and 12 
times throughout the experiment. The centers of any pair of dots were separated by a distance of between $5.43^{\circ}$ and $23.59^{\circ}$.

As in Experiment 1A, the irrelevant auditory stimuli of Experiment 3A consisted of digits in one block of trials, whereas they consisted of letters in the other block. The deviant item was always taken from the alternative category (i.e., deviants were digits in the block with TBI letters and vice versa). Experiment 3B used the auditory stimuli employed in Experiment 2. TBI sounds consisted of animal exemplars in one block (e.g., cat, dog, mouse, etc.), whereas they consisted of tool exemplars in the other block (e.g., drill, hammer, saw, etc.). On each standard trial, the auditory sequence was composed of eight words taken without replacement from the set of 16 words pertaining to each of these two semantic categories. On each deviant trial, a word taken from a new and different semantic category (e.g., colors, fruits, occupations, etc.) was inserted in the fifth position of the auditory sequence.

As in previous experiments, the acoustical/phonological qualities of the spoken stimuli must be performed in order to rule out an explanation of the categorical deviation effect in terms of acoustical factors. A matching of the spoken digits and letters for acoustical and phonological properties has already been provided in Experiment 1 (see Appendix A for more details). As for the category exemplars of Experiment 3B, the results of the acoustical/phonological analysis, presented in Appendix D, show that the deviant and standard exemplars did not differ with regard to their acoustical and phonological characteristics.

Procedure. Participants performed order reconstruction instead of serial recall. To begin a trial, participants had to press the spacebar. Four hundred milliseconds following the presentation of the last dot, all stimuli reappeared in their original location. Participants had to click on the dots using the mouse in the order in which they had been presented. Each item turned green once selected. No omissions nor repetitions were allowed. 


\section{Results}

Figure 5 shows the percentage of dots correctly reported according to the type of TBI sounds and the presence or not of a categorical deviant in Experiment 3A (left panel) and Experiment 3B (right panel). The pattern of results was similar to that observed in previous experiments: performance appeared lower in deviant trials, regardless of the type of auditory stimuli. Given that the type of sound was not equivalent across Experiments 3A (digits vs. letters) and 3B (animal vs. tool exemplars), results from the two experiments were first analyzed separately. The 2 (Type of sound) $\times 2$ (Deviation) repeated-measures ANOVA carried out on the data of Experiment 3A revealed a significant main effect of Deviation, $F(1,29)=7.09, p=.013$, $\eta_{\mathrm{p}}^{2}=.196, p_{\mathrm{BIC}}\left(\mathrm{H}_{0} \mid \mathrm{D}\right)=.175$, performance being poorer in the presence of a categorical deviation. Neither the main effect of Type of sound, $F(1,29)=2.57, p=.120, \eta_{\mathrm{p}}^{2}=.081$, $p_{\text {BIC }}\left(\mathrm{H}_{0} \mid \mathrm{D}\right)=.609$, nor the interaction were significant, $F(1,29)=0.26, p=.612, \eta_{\mathrm{p}}^{2}=.009$, $p_{\mathrm{BIC}}\left(\mathrm{H}_{0} \mid \mathrm{D}\right)=.833$. When performed on the data of Experiment 3B, the same ANOVA also revealed that the effect of Deviation was significant, $F(1,29)=16.52, p<.001, \eta_{\mathrm{p}}^{2}=.363$, $p_{\mathrm{BIC}}\left(\mathrm{H}_{0} \mid \mathrm{D}\right)<.001$, while that of Type of Sound $F(1,29)=0.09, p=.761, \eta_{\mathrm{p}}^{2}=.003, p_{\mathrm{BIC}}\left(\mathrm{H}_{0} \mid \mathrm{D}\right)=$ .881 , and the interaction were not, $F(1,29)=0.17, p=.682, \eta_{\mathrm{p}}^{2}=.006, p_{\mathrm{BIC}}\left(\mathrm{H}_{0} \mid \mathrm{D}\right)=.858$.

Next, we collapsed the data from the two types of sound in each experiment in order to enable the comparison of the impact of the two classes of distractors (alphanumerical stimuli vs. category exemplars) on the manifestation of the categorical deviation effect. A 2 (Class of distractors $) \times 2$ (Deviation) mixed ANOVA revealed a significant main effect of Deviation, $F(1$, $58)=19.74, p<.001, \eta_{\mathrm{p}}^{2}=.254, p_{\mathrm{BIC}}\left(\mathrm{H}_{0} \mid \mathrm{D}\right)<.001$, but not of Class of distractors, $F(1,58)=$ $0.85, p=.361, \eta_{\mathrm{p}}^{2}=.014, p_{\mathrm{BIC}}\left(\mathrm{H}_{0} \mid \mathrm{D}\right)=.834$. More importantly, the interaction between the two factors was not significant, $F(1,58)=0.001, p=.978, \eta_{\mathrm{p}}^{2}<.001, p_{\mathrm{BIC}}\left(\mathrm{H}_{0} \mid \mathrm{D}\right)=.916$, suggesting 
that both alphanumerical stimuli and category exemplars produced a categorical deviation effect of similar amplitude.
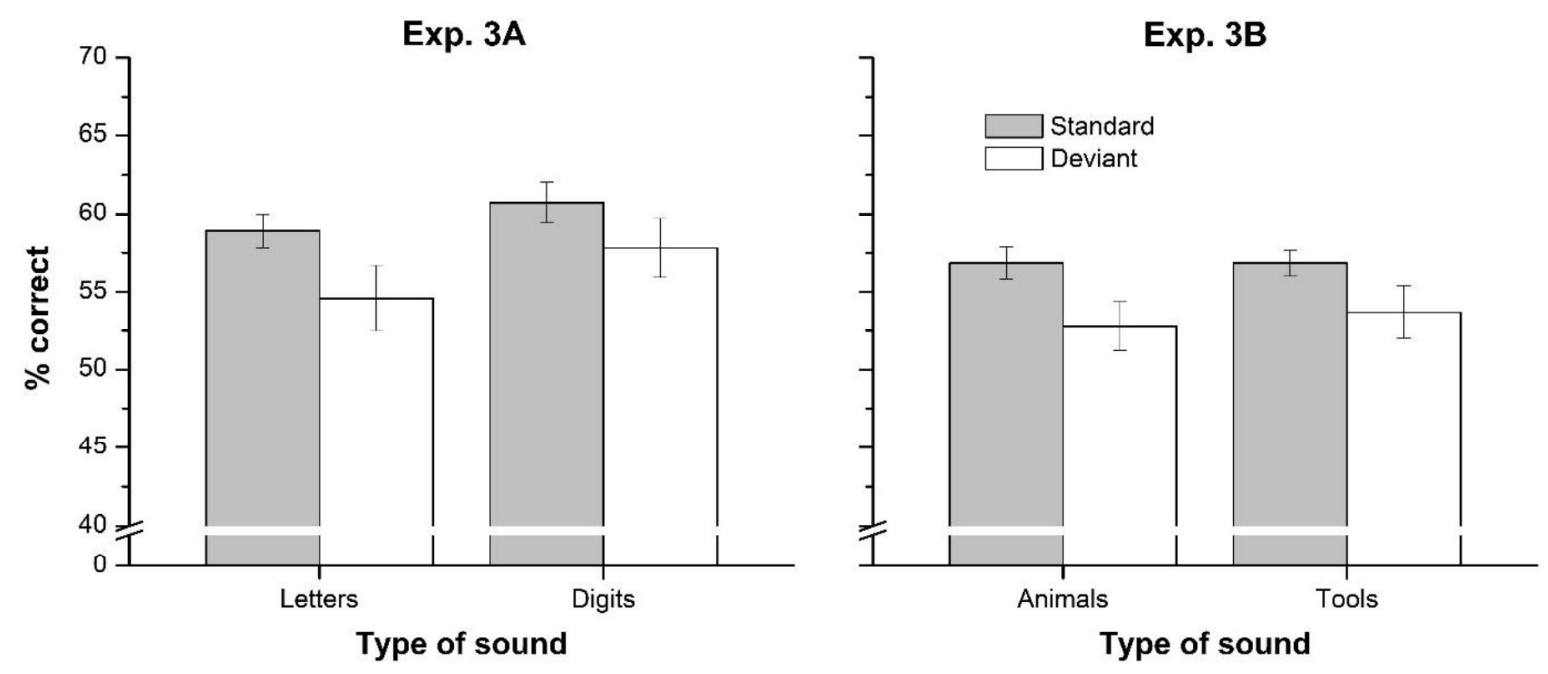

Figure 5. Mean percentage of dots correctly recalled in the four conditions of Experiment 3A (alphanumerical stimuli as to-be-ignored [TBI] material) and 3B (category exemplars as TBI material). Error bars represent $95 \%$ within-subject confidence intervals.

\section{Discussion}

Experiment 3 replicated the categorical deviation effect found in previous experiments in the context of the reconstruction of order of visuospatial stimuli. This effect was found using both semantically impoverished (Experiment 3A) and semantically rich TBI material (Experiment 3B). In fact, the two classes of stimuli produced the same amount of disruption. As in previous experiments, these deviation effects cannot be ascribed to acoustic origins given the equivalence of standard and deviant stimuli at both acoustic and phonological levels. Such findings, along with those observed in Experiments 1 and 2, suggest some form of equivalence between the distractive impact of a categorical change taking place in a semantically-rich setting (i.e. among category exemplars) and that occurring in a semantically-impoverished context (i.e. among alphanumerical stimuli). Given that, within the experimental setting of Experiment 3, the resort to verbal encoding strategies was deterred and the to-be-processed non-verbal items 
showed no association with the irrelevant spoken stimuli, it is virtually impossible to ascribe the disruptive impact of the category change observed here to a goal-related cognitive template (cf. Kiefer \& Martens, 2010) fostering the activation of verbal or semantic codes. Therefore, this result provides solid evidence that the detection of the irrelevant category switch took place preattentively, meaning that the content of the TBI auditory stream-whether semantically rich or not—was extracted in a goal-independent fashion.

\section{Interim Discussion}

In the first three experiments of the present study, we established a novel phenomenon whereby an unexpected and rare transient change in the semantic category of an irrelevant sequence of spoken items disrupted the serial recall or order reconstruction of visually-presented stimuli. From the tendency of the deviant item to intrude into recall (when possible) we can infer that such a disruption was the consequence of attention being involuntarily attracted toward that deviant and, thus, away from the focal task. This categorical deviation effect took place i) in the absence of any acoustical and/or phonological disparities between the deviant and standard stimuli, ii) despite the deviation conveying no particular signification to the participant, iii) in the absence of any relationship between the memoranda and the distracting information, iv) in the context of memory tasks that did not rely on, nor promote the activation of semantic or verbal codes, and v) regardless of the semantic richness of the TBI auditory items. Such a pattern of results therefore strongly suggests that this auditory distraction phenomenon is reliant neither on the semantic activation of auditory distractors being facilitated by the applied task set, nor on such distractors conveying task- or motivationally-relevant content. In fact, the present findings suggest that the attention response to the categorical mismatch embedded in the irrelevant sound ensued from an unintentional and goal-independent access to the meaning of the sound, 
consistent with the view that semantic processing is fully automatic. Yet, before concluding about the automaticity of semantic processing, two issues must be addressed.

First of all, we assumed that the irrelevant sound was processed preattentively, but it could have been possible for participants to nevertheless attend to the auditory stream even though they were instructed to ignore it. According to the attentional slippage hypothesis (Lachter et al., 2004), "slippage occurs when the attentional resources are allocated to irrelevant items as the result of inadequate control ("slips") of attention, causing these items to be identified" (p. 886). Lachter and colleagues (2004) posited that auditory processing is particularly prone to slippage of attention. If, in the present experimental setting, the focus of attention did occasionally wander-intentionally or not—away from the task-relevant material to the distractor channel, the analysis of the semantic content of the auditory sequence that is responsible for the categorical deviation effect observed could then be attributed to such brief attentional shifting toward-or co-monitoring of (see Muller-Gass, Macdonald, Schröger, Sculthorpe, \& Campbell, 2007; Röer et al., 2017a)—rather than to its preattentive extraction. From this standpoint, performance should be impaired on deviant trials when (a) slippage occurs during the presentation of the deviant AND at least one other item in the TBI sequence (most likely a temporally adjacent item), and (b) interference with the primary task is greater from two slippage-attended semantically unrelated items than from two slippage-attended semantically related items. In such a case, the occurrence of attention slippage in Experiments 1 to 3 would cast serious doubt about the view that the categorical deviation effect took place unintentionally. Although it may be impossible to fully rule out an attentional slippage account of the categorical deviation effect, critical aspects of the current methodology strongly suggest that the irrelevant auditory channel genuinely remained unattended. Slippage is likely to occur i) when the extent to which the focal task is demanding in terms of processing resources is low, ii) when 
distractors share some key features with task-relevant stimuli, iii) when the selected task set requires that some information about the irrelevant stimuli be processed, iv) when distractors remain present after the necessary information has been extracted from the relevant stimuli, and v) when irrelevant information can be exploited (e.g., as a cue) to perform the focal task (e.g., Lachter et al., 2004; Macken et al., 2003; Muller-Gass et al., 2007). The numerous empirical demonstrations of the semantic processing of irrelevant auditory information in the context of dichotic listening have been questioned because this paradigm suffers from several of these pitfalls (see Jones, 1999; Lachter et al., 2004). However, none of these situations are applicable to the current experimental setting. As discussed by Macken and colleagues (2003), some characteristics of the irrelevant sound paradigm employed in the present study act as safeguards against shifts of attention toward the auditory distractors. First, the serial recall of supraspan lists is a particularly demanding focal task. Second, unlike dichotic listening whereby two messages both presented aurally have to be separated, the discrimination between relevant and irrelevant material is easy in the irrelevant sound paradigm: not only are the TBR visual list and the auditory distractors presented in different sensory modalities but they also occur in different spatial locations. Moreover, care was taken in the present study to minimize the relationship between task-relevant and task-irrelevant information (Experiments 2-3) as well as the potential recourse to verbal coding strategies (Experiment 3). Therefore, there was little incentive for participants to attend to the auditory stream as spoken items did not provide any task-relevant information. Third, participants were told they would never be tested on the content of the sound and that no surprise test would be administered. Although the scope and extent of these safeguards suggest that the categorical deviation effect was not due to the focal processing of the irrelevant auditory material, some researchers argue that TBI sounds are routinely monitored for 
potentially important stimuli (Röer et al., 2017a; see also Hanczakowski et al., 2017).

Consequently, the attention-slippage hypothesis will be addressed in the remaining experiments.

A second issue that needs to be addressed before being able to conclude that the categorical deviation effect is mediated by the automatic activation of the content of the TBI material is whether, or not, this form of distraction is amenable to top-down control. This concerns the automaticity feature (un)controllable (Moors \& De Houwer, 2006, 2007). There is growing evidence that attentional capture by acoustical deviations is dependent upon such control (e.g., Horváth \& Bendixen, 2012; Hughes, Hurlstone, Marsh, Vachon, \& Jones, 2013; Parmentier \& Hebrero, 2013; Sussman, Winkler, \& Schröger, 2003). In stark contrast, the disruption produced by acoustically changing irrelevant information seems invulnerable to manipulations of top-down cognitive control (e.g., Hughes et al., 2013; Sörqvist, 2010). The immunity of this changing-state effect (cf. Jones, Madden, \& Miles, 1992) to cognitive control is due to the automatic nature of this form of auditory distraction: Disruption by changing-state sound is assumed to be a by-product of the obligatory perceptual organization of the auditory distractors (see Jones, Hughes, \& Macken, 2010; Jones \& Tremblay, 2000), an automatic process (cf. Bregman, 1990) that is not accessible to top-down control. Therefore, automatic forms of distraction, such as the changing-state effect, should not be affected by top-down cognitive control (for a discussion, see Hughes, 2014, and Jones et al., 2010). If the categorical deviation effect is truly an automatic effect, as the automatic semantic activation hypothesis would suggest, then it should be resistant to-i.e. should not be reduced by-top-down control manipulations. Given the functional similarities highlighted so far between the acoustical and categorical deviation effects (in both cases disruption arises from an unexpected deviation and is independent of the type of material used or task requirements), it is possible that the categorical version of the phenomenon would also be decreased by top-down manipulations. Any evidence 
that distraction by a categorical deviation can be decreased by top-down processes would indicate that semantic processing is controllable (cf. Moors \& De Houwer, 2006, 2007), supporting the view that meaning access is a conditionally automatic process. In contrast, the immunity of the categorical deviation effect to top-down influences would instead favor the view that semantic processing is fully automatic.

The next series of experiments was designed to test the susceptibility of the categorical deviation effect to top-down control. Shifts in top-down cognitive control settings were induced in response to increased task difficulty (Experiments 4 and 6) or foreknowledge about an imminent categorical deviation (Experiments 5-7). These top-down manipulations were selected not only on the basis of their modulatory impact on the disruptive effect of acoustical deviants (cf. Hughes et al., 2013), but also because of their potential to modulate-i.e. to either prevent or increase - the risk of attentional slippage. The following experiments share the commonality of using alphanumerical spoken items as TBI material so that any distraction effect cannot be explained in terms of acoustical/phonological factors (see Appendix A).

\section{Experiment 4}

The goal of this experiment was to examine whether conditions designed to promote focal-task engagement attenuate attentional capture by a categorical deviation embedded in the irrelevant sound sequence. Task (or cognitive) engagement refers to the level of concentration that is applied to the focal task and is assumed to reflect top-down cognitive control (e.g., Buetti \& Lleras, 2016; Halin, Marsh, Haga, Holmgren, \& Sörqvist, 2014; Hughes et al., 2013; Marsh, Sörqvist, \& Hughes, 2015; Sörqvist \& Marsh, 2015). The acoustical deviation effect (Hughes et al., 2013) as well as other forms of auditory distraction (e.g., Halin, 2016; Halin et al., 2014; Marsh, Sörqvist, \& Hughes, 2015; Marsh, Ljung et al., 2018; Marsh, Yang et al., 2018) are reduced or even abolished under conditions requiring a higher level of task engagement. It is 
argued that boosting the degree to which attention is actively engaged on the focal task tends to shield against distraction by making the locus of attention more steadfast (e.g., Halin et al., 2014; Sörqvist \& Marsh, 2015) and by attenuating the peripheral processing of task-irrelevant information (e.g., Halin, Marsh, \& Sörqvist, 2015; Marsh \& Campbell, 2016; Sörqvist, Dahlström, Karlsson, \& Rönnberg, 2016; Sörqvist, Stenfelt, \& Rönnberg, 2012; see also Buetti \& Lleras, 2016, for a similar notion in the visual domain). The need for concentration is largely determined by the difficulty of the focal task, although task engagement can also be mediated by other factors such as motivation and expertise (Ball, Threadgold, Solowiej, \& Marsh, 2018; Sörqvist \& Marsh, 2015).

Task engagement is typically influenced by manipulating task difficulty, for example the difficulty to perceptually discriminate relevant visual information (e.g., Halin et al., 2014; Hughes et al., 2013; Marsh, Sörqvist, \& Hughes, 2015; Marsh, Yang et al., 2018). For instance, Hughes and colleagues (2013) showed that when TBR items were made more transparent (i.e. less opaque) and embedded in static visual noise, the disruptive impact of acoustical deviants was abolished. In the present experiment, we sought to influence the level of task engagement by increasing task difficulty, specifically, by making it more difficult to perceptually identify the TBR items. As in Experiment 3, we wished to restrict the potential recourse to a verbal encoding strategy by using non-verbal TBR visual stimuli. However, there was no obvious way to reduce the discriminability of the visuospatial stimuli employed in Experiment 3. We therefore used pictures of unfamiliar faces as TBR material because the serial recall of such stimuli is arguably not based on verbal encoding strategies (see Smyth, Hay, Hitch, \& Horton, 2005). Stimulus discriminability was manipulated by varying the visual similarity of the TBR items. Based on the work of Smyth and colleagues, two sets of unfamiliar faces were created: TBR faces could either be similar or dissimilar. We reasoned that the greater task difficulty in the similar condition 
relative to the dissimilar condition would promote active focal-task engagement as a means of compensating for that increase in difficulty. If the categorical deviation effect is underpinned by the uncontrollable access to the meaning of the irrelevant sound and is therefore immune to topdown control, then such increased engagement should have no impact on the size of disruption. If, on the other hand, the effect is controllable and therefore open to top-down control, then increased task engagement should attenuate the categorical deviation effect.

An increase in the level of focal-task engagement should help to prevent the risk of attentional slippage. Indeed, the selective processing of task-relevant information is boosted in conditions where attention is tightly focused on the primary task (e.g., Hughes et al., 2013; Muller-Gass et al., 2007). Accordingly, the more steadfast the task engagement, the less likelihood of attention being drawn away from goal-related material. If the categorical deviation effect found in previous experiments was the result of the semantic activation of the auditory distractors caused by some — unintentional or deliberate—slips of attention toward the irrelevant sound, then the effect is expected to be reduced if not completely abolished under high taskengagement conditions. In such a case, the serial ordering of similar faces should be more resistant to the unexpected presence of a categorical deviant than the serial ordering of dissimilar faces. If, on the other hand, attentional slippage was not responsible for the disruption caused by categorical deviants, then face similarity should not influence the magnitude of the disruption.

\section{Method}

The method was identical to that employed in Experiment 1A, except as noted below.

Participants. Participants were 30 new volunteers with the same characteristics as those who took part in previous experiments. However, data from one participant was not considered since that participant failed to execute the task adequately. The final sample was therefore composed of 29 adults (21 women; mean age: 24.3 years). 
Materials. The TBR sequences now comprised seven male faces taken without replacement and arranged in a random order. The face images from CVL Face Database used in this work have been provided by the Computer Vision Laboratory, University of Ljubljana, Slovenia (Peer, Emeršič, Bule, Žganec-Gros, \& Štruc, 2014). Two sets of faces were generated. The 'similar' set was composed of seven faces that were similar in terms of age, hair and skin color, hairstyle, and face shape. This set corresponded to the set of similar faces illustrated in Figure 1A of Smyth and colleagues (2005). The 'dissimilar' set consisted of dissimilar faces according to the same criteria used to create the set of similar faces. Each TBR stimulus was displayed for $300 \mathrm{~ms}$ with an interstimulus interval of $600 \mathrm{~ms}$.

Auditory sequences were exclusively composed of spoken digits, except on deviant trials wherein a spoken letter replaced the fifth spoken digit. Given that TBR and TBI sequences were not of equal length, they were not synchronized. The onset of the first auditory item preceded the onset of the first visual item by $75 \mathrm{~ms}$.

Design and procedure. Two within-subject factors were manipulated: Task engagement (low vs. high) and Deviation (with and without deviant). In the low-engagement condition, participants performed order reconstruction of dissimilar faces. In the high-engagement condition, they did the same task but with the set of similar faces. Participants performed two blocks of 40 trials, one for each task-engagement condition, their order being counterbalanced across participants.

To begin a trial, participants clicked on a box containing the word "Start" which was located at the center of the screen. One second later, a fixation cross appeared for $1500 \mathrm{~ms}$. The first TBI sound was presented immediately after the disappearance of the fixation cross, shortly followed by the first TBR face. Three hundred milliseconds following the presentation of the seven faces, participants had to reconstruct the stimuli. In the response phase, all faces 
reappeared in random order with two faces on the top row, three on the middle row, and the last two on the bottom row. Participants had to click on the faces using the mouse in the order in which they had been presented. As faces were selected, they appeared horizontally at the bottom of the screen. Repetitions of the same item were possible, but no omissions were allowed.

\section{Results}

Figure 6 presents the percentage of faces correctly reported on standard and deviant trials according to the type of TBR stimuli. A 2 (Task engagement) $\times 2$ (Deviation) repeated-measures ANOVA carried out on these data revealed that order reconstruction was significantly poorer when task engagement was high, $F(1,28)=5.31, p=.029, \eta_{\mathrm{p}}^{2}=.159, p_{\mathrm{BIC}}\left(\mathrm{H}_{0} \mid \mathrm{D}\right)=.307$. The analysis also showed that performance was significantly lower in deviant trials than in standard trials, $F(1,28)=11.20, p=.002, \eta_{\mathrm{p}}^{2}=.286, p_{\mathrm{BIC}}\left(\mathrm{H}_{0} \mid \mathrm{D}\right)=.042$. This deviation effect was not modulated by task engagement, $F(1,28)=0.16, p=.697, \eta_{\mathrm{p}}^{2}=.006, p_{\mathrm{BIC}}\left(\mathrm{H}_{0} \mid \mathrm{D}\right)=.822$.

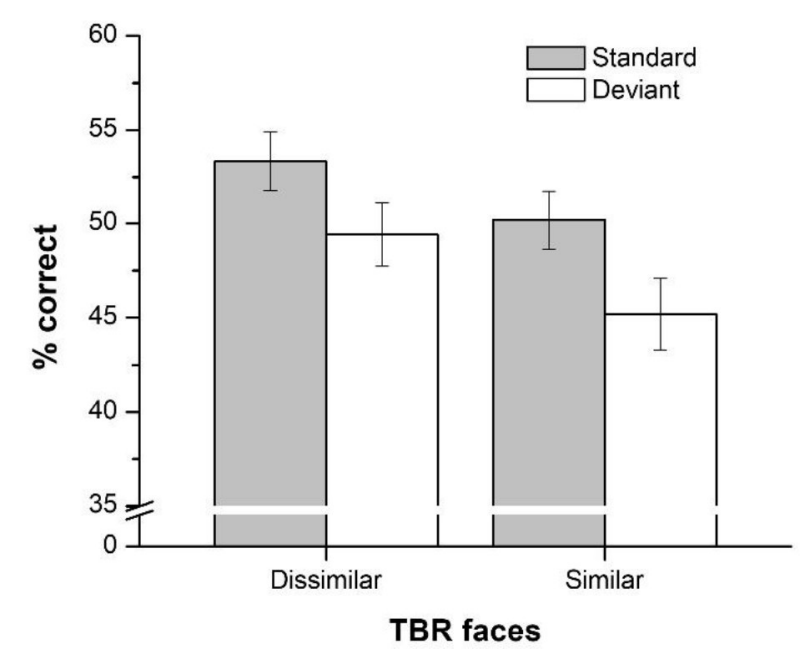

Figure 6. Mean percentage of faces correctly recalled in the four conditions of Experiment 4. Error bars represent $95 \%$ within-subject confidence intervals. 


\section{Discussion}

Experiment 4 demonstrated that the serial reconstruction of unfamiliar faces is disrupted by the introduction of a categorical deviant within the irrelevant auditory stream. Given that the task is arguably spatially oriented and does not rely on verbal encoding strategies (Smyth et al., 2005), this result provides further support that the categorical deviation effect is not contingent on the participant's task set (see also Experiment 3), consistent with a goal-independent view of the phenomenon. More important for the purpose of this experiment is the finding that the categorical deviation effect was not affected by the level of engagement required by the focal task. Indeed, the same amount of disruption was found whether participants had to recall similar or dissimilar faces. Since task engagement relies on cognitive control, associated with the voluntary commission of a greater task-directed investment (cf. Sörqvist \& Marsh, 2015), this finding suggests the categorical deviation effect is immune to cognitive control. Such a conclusion is clearly in accordance with the assumption that the extraction of the content of the TBI auditory sequence—necessary for the detection of a categorical deviation—takes place in an uncontrollable fashion. In the same vein, the fact that a reliable categorical deviation effect was found in the high-engagement condition argues against an attentional-slippage account of the effect (cf. Lachter et al., 2004; Röer et al., 2017a). Indeed, a higher engagement in the serial recall task should have helped hold the focus of attention in place thereby limiting slipping (or orienting) toward the auditory distractors.

\section{Experiment 5}

In order to test whether top-down processes can control the involuntary processing of the categorical change within the irrelevant sound, we examined in the current experiment the impact of forewarning participants of the imminent occurrence of a categorical deviant. There is evidence in the auditory distraction literature that when participants are told in advance that there 
would be a physical change within the auditory stimulation, the disruptive impact of such acoustical deviant is strongly reduced, if not abolished (e.g., Horváth \& Bendixen, 2012; Hughes et al., 2013; Parmentier \& Hebrero, 2013; Shelton, Elliott, Eaves, \& Exner, 2009; Sussman et al., 2003; but see Bell et al., 2017). This efficacy of a warning cue in shielding against deviance distraction derives from the high dependency of the disruptive power of an acoustical deviant upon its unexpectedness. There is indeed growing evidence that acoustical deviance captures attention because the deviant sound violates expectancies based on the invariance characterizing the recent auditory past (e.g., Bendixen et al., 2007; Nöstl, Marsh, \& Sörqvist, 2012; Parmentier, Elsley, Andrés, \& Barcelo, 2011; Schröger, Bendixen, Trujillo-Barreto, \& Roeber, 2007; Vachon et al., 2012; Winkler et al., 2009). This means that when the occurrence of a deviant can be anticipated, either because the deviant sound happens in a predictable fashion (e.g., Parmentier et al., 2011; Vachon et al., 2012) or it is forewarned (e.g., Hughes et al., 2013; Parmentier \& Hebrero, 2013; Shelton et al., 2009), it can be actively—i.e. voluntarily-incorporated into a predictive model of the imminent auditory sequence (cf. Bendixen et al., 2007; Winkler et al., 2009), so that the acoustical deviation would no longer constitute a cognitive violation.

Experiment 5 examined whether foreknowledge of a categorical deviation exerts a comparable effect to that of an acoustical deviation. In the context of the serial recall of dissimilar faces, participants' anticipation of a categorical change within the irrelevant sound was manipulated by providing them with a warning, or not, before each trial as to whether the incoming sequence of spoken digits would contain a (deviant) spoken letter or not. If the categorical deviation effect is not susceptible to top-down influences, as suggested by the results of Experiment 4, then such a warning should not affect the disruption caused by the insertion of a (deviant) letter among spoken digits. If, on the other hand, the effect is open to top-down control, 
then forewarning the change of category within the auditory stream should help to suppress its processing (cf. Sussman et al., 2003), hence preventing disruption.

The manipulation of foreknowledge regarding the deviant could also help to resolve the potential implication of attentional slippage in the elicitation of the categorical deviation effect. According to Lachter and colleagues (2004), slippage can take place out of curiosity. Knowing that a special (deviant) stimulus is about to occur could therefore increase the propensity to allocate attention to the auditory distractors. Thus, if attentional slippage toward the irrelevant sound is responsible for the categorical deviation effect, then more disruption is expected under conditions in which the occurrence of the categorical deviant is known in advance.

\section{Method}

The method was identical to that employed in Experiment 4, except as noted below.

Participants. Participants were 45 new volunteers (34 women; mean age: 26.2 years) with the same characteristics as those who took part in previous experiments.

Materials. The TBR lists were always conveyed using the dissimilar faces ensemble (i.e., low task engagement) of Experiment 4.

Design and procedure. The experiment employed a 2 (Warning: with and without) $\times 2$ (Deviation: with and without) within-subject design. Warning condition was blocked and it was counterbalanced across participants. Each block consisted in 32 standard trials and 8 deviant trials, making 80 trials in all. In one block, the deviant sequences occurred on Trials 5, 8, 15, 21, $26,30,36$, and 39 , whereas in another block they occurred on Trials $4,10,14,19,25,28,35$, and 40. These two deviant-trial schedules were rotated over the no-warning and warning blocks.

At the beginning of the warning block, subjects were instructed they would be provided before each trial with a warning informing them of the presence or not of a spoken letter (the "deviant") embedded among the spoken digits composing the TBI sequence. They were also told 
that on "deviant" trials, they should try hard to ignore that (deviant) letter. In the no-warning block, a box containing the word "Start" presented in black font appeared in the central screen position at the start of each trial, and participants had to click on the box using the mouse to start the trial. In the warning block, this box contained the message "No Deviant" presented in black font on standard trials, whereas the message "Deviant" was presented in red font on deviant trials. The first TBR and TBI items were presented concurrently $250 \mathrm{~ms}$ after participants clicked on the pre-trial message with the mouse. Trials were self-paced to ensure that participants would not miss the pre-trial messages. In each block, participants performed two practice trials before completing the experimental trials: In the no-warning block, these consisted of two standard trials whereas in the warning block, they consisted of one standard and one deviant trials.

\section{Results}

Figure 7 shows the percentage of faces correctly reordered in standard and deviant trials as a function of the provision of a pre-trial warning or not. A 2 (Deviation) $\times 2$ (Warning) repeated-measures ANOVA performed on these data showed a significant main effect of Deviation, $F(1,44)=8.58, p=.005, \eta_{\mathrm{p}}^{2}=.163, p_{\mathrm{BIC}}\left(\mathrm{H}_{0} \mid \mathrm{D}\right)=.105$, confirming the presence of the categorical deviation effect. However, neither the main effect of Warning, $F(1,44)=0.31, p=$ $.579, \eta_{\mathrm{p}}^{2}=.007, p_{\mathrm{BIC}}\left(\mathrm{H}_{0} \mid \mathrm{D}\right)=.851$, nor the interaction were significant, $F(1,44)=0.02, p=.896$, $\eta_{\mathrm{p}}^{2}<.001, p_{\mathrm{BIC}}\left(\mathrm{H}_{0} \mid \mathrm{D}\right)=.869$.

\section{Discussion}

The results of Experiment 5 replicated those of Experiment 4 by showing a categorical deviation effect in the context of the serial recall of unfamiliar faces, providing further evidence that the effect can take place under conditions where the established task set does not foster the activation of verbal or semantic codes. More importantly, this deviation effect remained 
unaffected by the foreknowledge of the upcoming categorical deviant. The acoustical deviation effect tends to vanish when the occurrence of the physical change can be anticipated (e.g., Hughes et al., 2013). The present findings revealed that such foreknowledge about, this time, a change of category did not prevent such a change from disrupting performance. Consistent with the conclusion of Experiment 4, such findings suggest that the categorical deviation effect is not amenable to top-down control. Moreover, the failure to find increased disruption in a condition where the presence of an atypical stimulus (here, a letter among digits) in an otherwise homogeneous irrelevant stream was forewarned provides evidence against the attentionalslippage origins of the effect. Indeed, foreknowledge of the imminent occurrence of a special distractor is likely to promote the processing of the distractor stream out of curiosity (cf. Lachter et al., 2004).

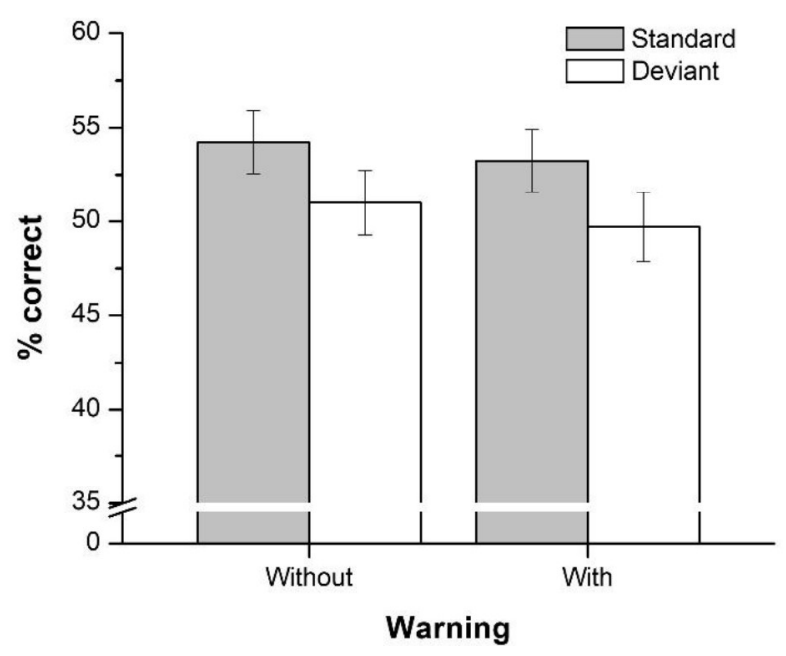

Figure 7. Mean percentage of faces correctly recalled in the four conditions of Experiment 5. Error bars represent $95 \%$ within-subject confidence intervals.

Overall, the results of Experiment 5, along with those of Experiment 4, were consistent with the view that the categorical deviation effect arises from the uncontrollable processing of the content of the irrelevant auditory material. Indeed, the observations that reliable disruption 
took place in conditions of high task engagement (Experiment 4) or deviant foreknowledge (Experiment 5) suggest that the categorical deviation effect is independent of top-down control, contrary to its acoustical counterpart (see Hughes et al., 2013). Yet these empirical demonstrations that the effect is not susceptible to the manipulation of top-down factors also rely on the observation of null interaction effects. Although Bayes factor analyses provided positive support for the null hypothesis (the probability that the data favor the null hypothesis for the interaction were .822 and .869 in Experiments 4 and 5, respectively), these results need to be replicated. It cannot be excluded, for example, that the use of similar faces was insufficient to increase the level of engagement in the serial recall task enough to observe a significant impact of task engagement on the amount of disruption produced by the categorical deviant (see Discussion of Experiment 4). Consequently, Experiment 6 was designed to test the influence of task engagement and foreknowledge on the categorical deviation effect within an experimental setting known for its successful manipulation of these top-down factors.

\section{Experiment 6}

Since in Experiment 4 similar faces were significantly more difficult to recall than dissimilar faces (see also Smyth et al., 2005), we assumed that our task-engagement manipulation was effective. Yet the supposition that the processing of similar faces was more demanding than that of dissimilar faces could only be inferred from the results. Typically, task engagement is boosted to compensate for increased task difficulty. Consistent with this idea of a compensatory shift in task engagement are the demonstrations that increasing task difficulty does not necessarily produce drops in performance (e.g., Hughes et al., 2013; Marsh, Ljung et al., 2018; Marsh, Sörqvist, \& Hughes, 2015; see also Ball et al., 2018). In fact, the reduction of the level of recall performance with increasing task difficulty observed in Experiment 4 would be expected if task engagement was not sufficiently boosted (see, e.g., Eggemeier, Crabtree, \& 
LaPointe, 1983). It is therefore possible that the increase in similarity between the faces failed to translate into a proper increase in task engagement. This possibility will be addressed in the current experiment.

In an attempt to replicate the apparent immunization of the categorical deviation effect to top-down factors revealed in Experiments 4 and 5, the current experiment capitalized on the methodology and design employed by Hughes and colleagues (2013) in their second experiment where they established the sensitivity of the acoustical version of the phenomenon to both task difficulty and deviant foreknowledge. Accordingly, participants performed serial recall of digits while ignoring sequences of spoken letters, similarly to Experiment 1A. This time, task engagement and deviant foreknowledge were manipulated in a within-subject fashion so as to independently evaluate the impact of each factor within the same experiment. This also allowed for the combination of the two factors so as to create a condition that would maximize the potential power of top-down influences. An outcome whereby deviant disruption takes place even under a high-engagement condition with deviant forewarning would strongly militate against the idea that the categorical deviation effect is amenable to top-down control.

In order to influence the degree to which attention would need to be actively engaged on the serial recall task, task difficulty was increased this time by perceptually degrading the TBR items so that they would be more effortful to identify. In the high-engagement condition, each digit in the TBR list was made transparent and embedded in static visual noise (cf. Hughes et al., 2013; Marsh, Sörqvist, \& Hughes, 2015; Parmentier, Elford, Escera, Andrés, \& San Miguel, 2008; Yi, Woodman, Widders, Marois, \& Chun, 2004), as illustrated in the right panel of Figure 8. This was compared with a low-engagement condition in which each TBR digit was presented in the usual, non-degraded fashion (see left panel of Figure 8). Hughes and colleagues (2013) showed that such perceptual degradation of the stimuli extends the time required for their 
identification, confirming that this manipulation is efficient in increasing task difficulty. Moreover, Marsh, Sörqvist, and Hughes (2015) showed that embedding TBR items in visual static noise improves source monitoring-i.e. reduces processing of the irrelevant auditory material—via a front-end control mechanism. In the same way as in Experiment 5, deviant foreknowledge was manipulated by providing a warning, or not, about the presence of a categorical change in the upcoming auditory sequence. More specifically, before each trial of the warning condition, participants were informed of whether, or not, a spoken digit would be embedded in the irrelevant sequence of spoken letters.

The use of a deviant item drawn from the same category as the visual memoranda (i.e. digits) allowed for the application of the deviant intrusion technique employed in Experiment 1. As a reminder of this technique, each TBR list was composed of all but one of the set of digits 19 and the missing digit was systematically presented auditorily as the deviant item on deviant trials. The application of such a procedure conferred the advantage of testing the impact of topdown manipulations not only on a single measure but instead on two distinct dependent variables, namely serial recall performance and deviant-intrusion errors. For instance, Marsh, Sörqvist, and Hughes (2015) found not only an improvement of the free recall of category exemplars in the presence of semantically-related irrelevant speech, but also a reduction in the number of intrusions of spoken distractors through the degradation manipulation employed here. By measuring distraction by categorical deviants in two different ways in an experiment that factorially combined a manipulation of task engagement with a manipulation of foreknowledge, we hoped to undertake a more sensitive test of the influence of top-down factors on the categorical deviation effect.

\section{Method}

The method was identical to that employed in Experiment 1A, except for what follows. 


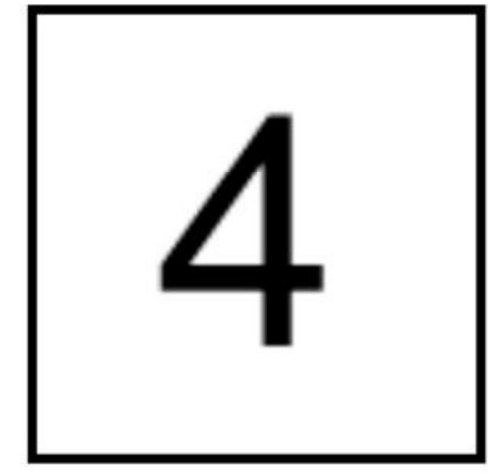

Non degraded

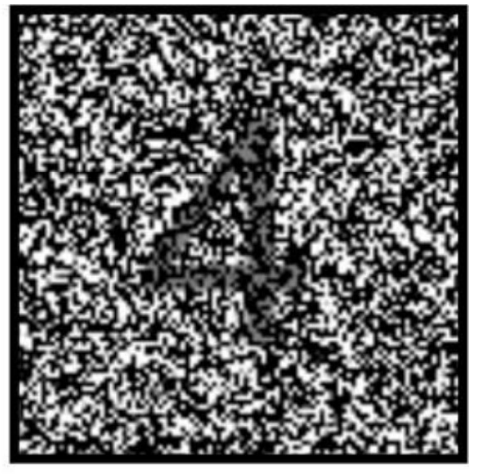

Degraded

Figure 8. Illustration of how the to-be-remembered digits appeared in the low task engagement (non degraded) and high task engagement (degraded) conditions of Experiment 6.

Participants. Participants were 24 new volunteers (19 women; mean age: 23.5 years) with the same characteristics as those who took part in previous experiments.

Materials. Each TBR sequence was composed of eight digits sampled without replacement from the set $1-9$, arranged in a quasi-random order with the constraint that there were no ascending or descending runs of more than two digits. Two versions of the 9-digit set were created and saved as bitmap files on the computer controlling stimulus presentation. In the non-degraded set, digits were clearly visible, presented in black against a white background (see left panel of Figure 8). TBR lists of non-degraded digits was used in the low-engagement condition. In the degraded set, the digits were degraded by setting the transparency of the item to $50 \%$, and by adding a visual mask comprising static Gaussian visual noise (400\%) over the item using the Adobe Photoshop software (see right panel of Figure 8). The degraded set of TBR digits was used in the high-engagement condition.

Auditory sequences were composed of eight letters randomly selected from the set B, F, $\mathrm{H}, \mathrm{K}, \mathrm{M}, \mathrm{Q}, \mathrm{R}, \mathrm{X}$, and Z. On deviant trials, the fifth letter was replaced by a spoken digit. This deviant digit corresponded to the digit that was absent from the TBR visual sequence. 
Design and procedure. The experiment had a 2 (Deviation: no deviant vs. deviant) $\times 2$ (Task engagement: low vs. high) $\times 2$ (Warning: with vs. without) within-subject design. Whereas Deviation and Task engagement varied from trial to trial in a random fashion, Warning was blocked. Participants completed two experimental blocks, one in the no-warning condition and the other in the warning condition. The order of these blocks was counterbalanced across participants. Each block consisted of 80 standard trials (40 low-engagement trials and 40 high task-engagement trials) and 12 deviant trials (6 low-engagement trials and 6 high-engagement trials), making 184 trials in all. In one block, the deviant sequences occurred on Trials 10, 21, 24, $33,38,45,55,66,69,78,83$, and 90 , whereas in the other block, they occurred on Trials 5,8 , $18,27,35,41,50,53,63,72,80$, and 86 . These two deviant-trial schedules were rotated over the no-warning and warning blocks.

At the beginning of the warning block, participants were instructed they would be provided before each trial with a warning informing them of the presence or not of a spoken digit (the "deviant") embedded among the spoken letters composing the TBI sequence. They were also informed that on "deviant" trials, they should try hard to ignore that (deviant) digit. In the no-warning block, a box containing the word "Start" presented in black font appeared in the central screen position at the start of each trial, and participants had to click on the box using the mouse to start the trial. In the warning block, this box contained the message "No Deviant" presented in black font on standard trials, whereas the message "Deviant" was presented in red font on deviant trials. The TBR and TBI sequences started simultaneously $250 \mathrm{~ms}$ after the participant clicked on the message box with the mouse. Trials were self-paced to make sure that participants would not miss the pre-trial message. There was no time limit for participants to write down their responses in the answer booklet. When participants were ready to go to the next trial, they clicked anywhere on the screen using the mouse. 
Participants began the no-warning block with four practice trials consisting of two lowengagement and two high-engagement standard trials. Before the warning block, they also completed two practice trials in each task-engagement condition, but this time one of the two trials was accompanied by a TBI sequence containing a categorical deviant. The two experimental blocks were administered on separate days given the taxing nature of the serial recall task. Each experimental session took approximately 45 minutes to complete.

\section{Results}

Serial recall. Figure 9 presents serial recall performance for non-degraded and degraded TBR digits on both standard and deviant trials of the no-warning (left panel) and the warning (right panel) conditions of Experiment 6. Recall appeared to be poorer on deviant trials than on standard trials regardless of task difficulty and the presence or absence of a warning cue. This pattern of results was confirmed by a 2 (Deviation) $\times 2$ (Task engagement $) \times 2$ (Warning) repeated-measures ANOVA performed on these data. Whereas the main effects of Task engagement, $F(1,23)=0.07, p=.789, \eta_{\mathrm{p}}^{2}=.003, p_{\mathrm{BIC}}\left(\mathrm{H}_{0} \mid \mathrm{D}\right)=.825$, and Warning, $F(1,23)=$ $0.01, p=.937, \eta_{\mathrm{p}}^{2}<.001, p_{\mathrm{BIC}}\left(\mathrm{H}_{0} \mid \mathrm{D}\right)=.830$, were not significant, that of Deviation was significant, $F(1,23)=25.97, p<.001, \eta_{\mathrm{p}}^{2}=.530, p_{\mathrm{BIC}}\left(\mathrm{H}_{0} \mid \mathrm{D}\right)<.001$, indicating the presence of a categorical deviation effect. Importantly, this Deviation effect did not interact with neither Task engagement, $F(1,23)=0.44, p=.514, \eta_{\mathrm{p}}^{2}=.019, p_{\mathrm{BIC}}\left(\mathrm{H}_{0} \mid \mathrm{D}\right)=.796$, nor Warning, $F(1,23)=$ $0.32, p=.580, \eta_{\mathrm{p}}^{2}=.014, p_{\mathrm{BIC}}\left(\mathrm{H}_{0} \mid \mathrm{D}\right)=.806$, nor the interaction of Task engagement and Warning, $F(1,23)=0.68, p=.417, \eta_{\mathrm{p}}^{2}=.029, p_{\mathrm{BIC}}\left(\mathrm{H}_{0} \mid \mathrm{D}\right)=.786$, suggesting that the magnitude of the categorical deviation effect was not influenced by the two manipulated top-down factors. 

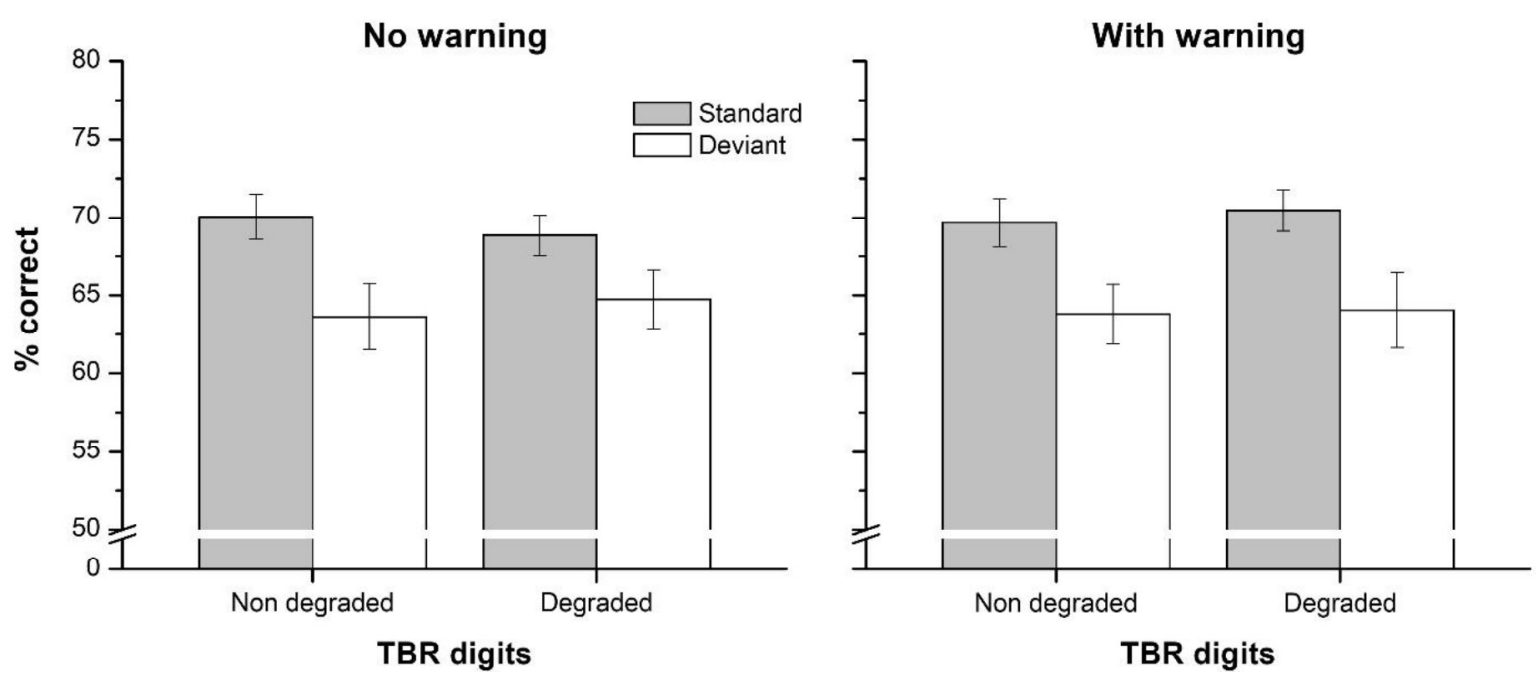

Figure 9. Mean percentage of items correctly recalled in standard and deviant trials under low (non-degraded digits) and high (degraded digits) task engagement for the no-warning (left panel) and the warning conditions (right panel) of Experiment 6. Error bars represent 95\% within-subject confidence intervals.

Intrusions. As in Experiment 1A, the percentage of intrusion errors-i.e. the incorrect recall of the digit that was 'missing' from the TBR list—was compared between standard trials, in which TBI sequences were exclusively composed of spoken letters, and deviant trials, in which a deviant digit corresponding to the identity of the missing item was inserted in the irrelevant auditory stream. Figure 10 shows the percentage of missing-item intrusions for standard and deviant trials according to the level of task engagement and the presence or not of pre-trial warnings. A visual inspection of the figure revealed that intrusion errors were more likely in deviant trials than in standard trials in every condition. These data were submitted to a 2 $($ Deviation $) \times 2($ Task engagement $) \times 2($ Warning $)$ repeated-measures ANOVA, which revealed no significant main effect of Task engagement, $F(1,23)=0.01, p=.917, \eta_{\mathrm{p}}^{2}<.001, p_{\mathrm{BIC}}\left(\mathrm{H}_{0} \mid \mathrm{D}\right)=$ .830 , or Warning, $F(1,23)=0.06, p=.804, \eta_{\mathrm{p}}^{2}=.003, p_{\mathrm{BIC}}\left(\mathrm{H}_{0} \mid \mathrm{D}\right)=.825$. The significant main effect of Deviation, $F(1,23)=35.03, p<.001, \eta_{\mathrm{p}}^{2}=.604, p_{\mathrm{BIC}}\left(\mathrm{H}_{0} \mid \mathrm{D}\right)<.001$, confirmed the increased number of intrusions in deviant trials. Importantly, this higher tendency for the 
intrusion of the missing item when presented as the categorical deviant was not modulated by neither Task engagement, $F(1,23)=0.40, p=.531, \eta_{\mathrm{p}}^{2}=.017, p_{\mathrm{BIC}}\left(\mathrm{H}_{0} \mid \mathrm{D}\right)=.800$, nor Warning, $F(1,23)=0.79, p=.781, \eta_{\mathrm{p}}^{2}=.003, p_{\mathrm{BIC}}\left(\mathrm{H}_{0} \mid \mathrm{D}\right)=.824$, nor the interaction of these two factors, $F(1,23)=1.30, p=.265, \eta_{\mathrm{p}}^{2}=.054, p_{\mathrm{BIC}}\left(\mathrm{H}_{0} \mid \mathrm{D}\right)=.717$.
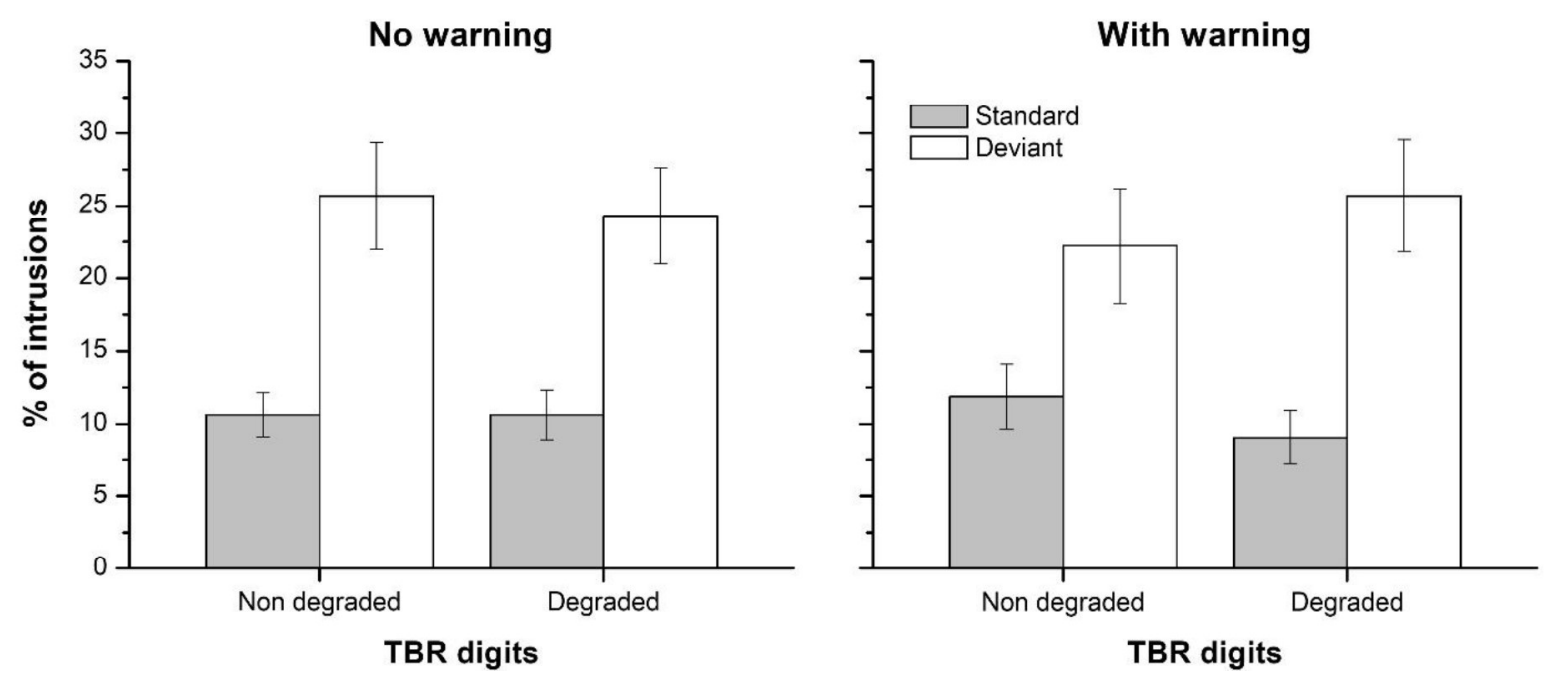

Figure 10. Mean percentage of intrusions of the missing item when absent from the trial (standard) and when presented as the auditory deviant (deviant) under low (non-degraded digits) and high (degraded digits) task engagement for the no-warning (left panel) and the warning conditions (right panel) of Experiment 6. Error bars represent 95\% within-subject confidence intervals.

\section{Discussion}

Adopting the method and design used by Hughes and colleagues (2013), Experiment 6 replicated the findings of Experiments 4 and 5 with regard to the failure of top-down factors to modulate the categorical deviation effect. Indeed, the disruptive impact on serial recall of a change of category within the irrelevant auditory channel remained unaltered by both an increase in the level of task engagement induced by the perceptual degradation of TBR stimuli, and the foreknowledge of the imminent encounter with the deviant event. Not only did such top-down manipulations fail to reduce the distraction caused by the categorical deviation, they also did not diminish the propensity of the deviant item to erroneously intrude into the recalled list. Such 
results revealed that distraction and intrusions were neither diminished in a condition of high task engagement designed to discourage the allocation of attentional resources to the irrelevant sound nor increased by foreknowledge that could attract attention (out of curiosity) toward an incoming special distractor. Taken together, these findings provide further support against an attentionalslippage view of the categorical deviation effect. In fact, this new demonstration that the phenomenon is independent from top-down control militates in favor of a conceptualization of this form of distraction in terms of uncontrollable content analysis of the irrelevant sound.

In contrast with Experiment 4, the current experiment did not reveal any drop in performance provoked by the decrease in perceptual discriminability of the TBR items. This result, however, is consistent with previous studies showing that manipulations designed to increase perceptual demand, such as the perceptual degradation of task-relevant material (Hughes et al., 2013; Marsh, Sörqvist, \& Hughes, 2015) or the use of a disfluent-i.e. difficultto-read-font (Halin et al., 2014; Marsh, Ljung et al., 2018) can reduce or even abolish auditory distraction without having an impact on task performance. According to Sörqvist and Marsh (2015), "when task difficulty is high, people make a compensatory upward shift in concentration in order to maintain their desired level of performance. As a result, people's locus of attention becomes more steadfast" (p. 269). The absence of effect on task performance can therefore be taken as evidence that the perceptual degradation of the TBR stimuli successfully boosted the level of focal task engagement. In any case, we failed to find an impact of perceptual discriminability on the behavioral manifestation of the categorical deviation effect whether focal task performance was impaired (Experiment 4) or not (current experiment) by such a manipulation, suggesting that the phenomenon is truly immune to top-down cognitive control.

Despite the large number of experiments demonstrating that the foreknowledge of an imminent acoustical irregularity can shield against its distractive power (e.g., Horváth \& 
Bendixen, 2012; Hughes et al., 2013; Parmentier \& Hebrero, 2013; Shelton et al., 2009; Sussman et al., 2003), the present study failed to extend such a finding to categorical disparities. While both acoustical and categorical deviations constitute a digression from some invariance within the auditory environment, they nevertheless differ fundamentally on the nature of such deviance. Since content is at the heart of a categorical deviation, one could argue that simply forewarning about the imminent occurrence of some discrepancy in the content of the stimulation with no more detail about that content may not be sufficient to preclude such incongruity from causing distraction. In other words, it is possible that the failure to observe an impact of categorical deviant foreknowledge in Experiments 5 and 6 ensued from the use of a forewarning that was not specific enough (cf. Röer, Bell, \& Buchner, 2015) to efficiently reduce the unexpectedness of the deviant event. This issue was addressed in the next experiment.

\section{Experiment 7}

The goal of this experiment was to further test the impact of foreknowledge on the categorical deviation effect by examining the potential efficacy of forewarning the identity of the deviant item in reducing the disruption caused by the deviant. Such specific foreknowledge may be necessary to build up a proper mental representation of an upcoming categorical deviation within the irrelevant channel. Röer and colleagues (2015; see also Bell et al., 2017) showed that increasing the specificity of pre-trial warnings can help to further reduce the amount of distraction caused by irrelevant speech, especially for meaningful distractor material. By informing participants before each deviant trial about the exact content of the incongruity they were going to hear, we tried to maximize the chances of finding a significant foreknowledge effect. Therefore, we compared the categorical deviation effect in the absence of any information about the upcoming deviation to two forewarning conditions. In the 'unspecific' condition, the same (unspecific) warning about the imminent occurrence (or not) of a categorical change (e.g., 
"a digit will be inserted among the letters in the irrelevant auditory sequence") as in previous experiments was presented before every trial. In the 'specific' forewarning condition, the pretrial message took the form of a 'specific' warning about both the occurrence and, more importantly, the identity of the deviant event within the upcoming auditory stream (e.g., "a spoken ' 8 ' will be inserted among the spoken letters of the irrelevant auditory sequence"). It is noteworthy that, as in previous experiments, the deviant was always presented in the same position within the auditory sequence, making its occurrence even more predictable in the forewarning conditions. If the failure to observe foreknowledge effects in Experiments 5 and 6 was attributable to a lack of specificity of the pre-trial warning cues employed, then the use of specific forewarnings should help reduce the disruption found in deviant trials. If, on the other hand, the absence of foreknowledge effects truly reflected the insensitivity of the categorical deviation effect to top-down control, then neither specific or unspecific warnings should be endowed with the power to shield against the disruptive impact of the deviant event.

\section{Method}

The method was identical to that employed in Experiment 6, except for the following differences. Participants were 36 new volunteers (21 women; mean age: 24.5 years) with the same characteristics as those who took part in previous experiments. They performed the serial recall task on non-degraded digits only. The experiment employed a 2 (Deviation: with or without deviant) $\times 3$ (Type of warning: none, unspecific and specific) within-subject design. The type of pre-trial warning was manipulated across three different blocks of trials, and the order of these blocks was counterbalanced across participants. Each block consisted of 32 standard trials and 8 deviant trials. In one block, the deviant sequences occurred on Trials $6,9,15,19,23,30$, 35, and 39. In another block, they occurred on Trials $5,10,13,17,23,30,32,40$, whereas in the 
remaining block, they occurred on Trials $6,9,13,20,22,29,33$, and 36. Those three devianttrial schedules were rotated over the three pre-trial warning conditions.

After being given general instructions about the task to be performed, participants began the experimental session with two standard practice trials. Before each experimental block, participants were given directions that were specific to the forthcoming set of trials. The nowarning block was preceded by a reminder of the serial recall task and of the need to ignore the irrelevant spoken sequences that were presented simultaneously. The instructions given prior to the unspecific warning block went further by indicating to participants that they would be provided with a pre-trial warning informing them of the presence or not of a spoken digit (the "deviant") embedded among the spoken letters composing the TBI sequence. They were also informed that on "deviant" trials, they should try hard to ignore that (deviant) digit. The same instructions were given before the specific warning block, but participants were also informed that on deviant trials, they would be provided with the identity of the "deviant" digit. The experimental session took approximately 50 minutes to complete.

In the no-warning block, a box containing the word "Start" appeared in the central screen position at the start of each trial, and participants had to click on the box using the mouse to start the trial. In the unspecific warning block, this box contained the message "No Deviant" presented in black font on standard trials, whereas the message "Deviant" was presented in red font on deviant trials. In the specific warning block, the red message also included the identity of the deviant digit (e.g., if the upcoming deviant was 8, the message was "Deviant 8").

\section{Results}

Serial recall. Figure 11 shows serial recall performance on both standard and deviant trials for each of the three types of warning. Recall appeared to be poorer on deviant than on standard trials regardless of the condition. This pattern of results was confirmed by a 2 
(Deviation) $\times 3$ (Type of warning) repeated-measures ANOVA performed on these data. The main effect of Deviation was significant, $F(1,35)=28.16, p<.001, \eta_{\mathrm{p}}^{2}=.446, p_{\mathrm{BIC}}\left(\mathrm{H}_{0} \mid \mathrm{D}\right)<$ .001 , pointing to the presence of the categorical deviation effect. However, neither the main effect of Type of warning, $F(2,70)=0.44, p=.648, \eta_{\mathrm{p}}^{2}=.012, p_{\mathrm{BIC}}\left(\mathrm{H}_{0} \mid \mathrm{D}\right)=.983$, nor the interaction were significant, $F(2,70)=0.45, p=.640, \eta_{\mathrm{p}}^{2}=.013, p_{\mathrm{BIC}}\left(\mathrm{H}_{0} \mid \mathrm{D}\right)=.983$, suggesting that the deviation effect was not modulated by the provision of a warning before each trial, whether this warning was specific or unspecific.

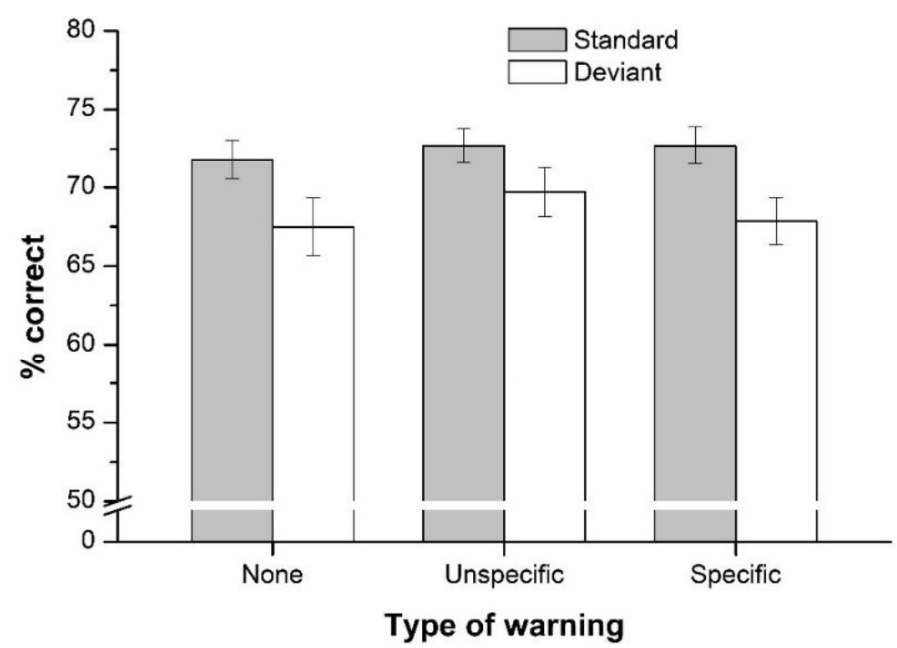

Figure 11. Results from Experiment 7: Mean percentage of items correctly recalled in standard and deviant trials as a function of the type of warning. Error bars represent $95 \%$ within-subject confidence intervals.

Intrusions. Figure 12 presents the percentage of intrusion errors in standard and deviant trials according to the type of warning. Intrusions of the missing item seemed more frequent in deviant trials than in standard trials, regardless of the type of warning provided. The 2 $($ Deviation $) \times 3$ (Type of warning) repeated-measures ANOVA performed on intrusion errors showed that whereas the main effect of Deviation was significant, $F(1,35)=43.51, p<.001, \eta_{\mathrm{p}}^{2}$ $=.554, p_{\mathrm{BIC}}\left(\mathrm{H}_{0} \mid \mathrm{D}\right)<.001$, the main effect of Type of warning, $F(2,70)=0.55, p=.581, \eta_{\mathrm{p}}^{2}=$ 
$.015, p_{\mathrm{BIC}}\left(\mathrm{H}_{0} \mid \mathrm{D}\right)=.978$, and the interaction were not significant, $F(2,70)=0.26, p=.772, \eta_{\mathrm{p}}^{2}=$ $.007, p_{\mathrm{BIC}}\left(\mathrm{H}_{0} \mid \mathrm{D}\right)=.989$.

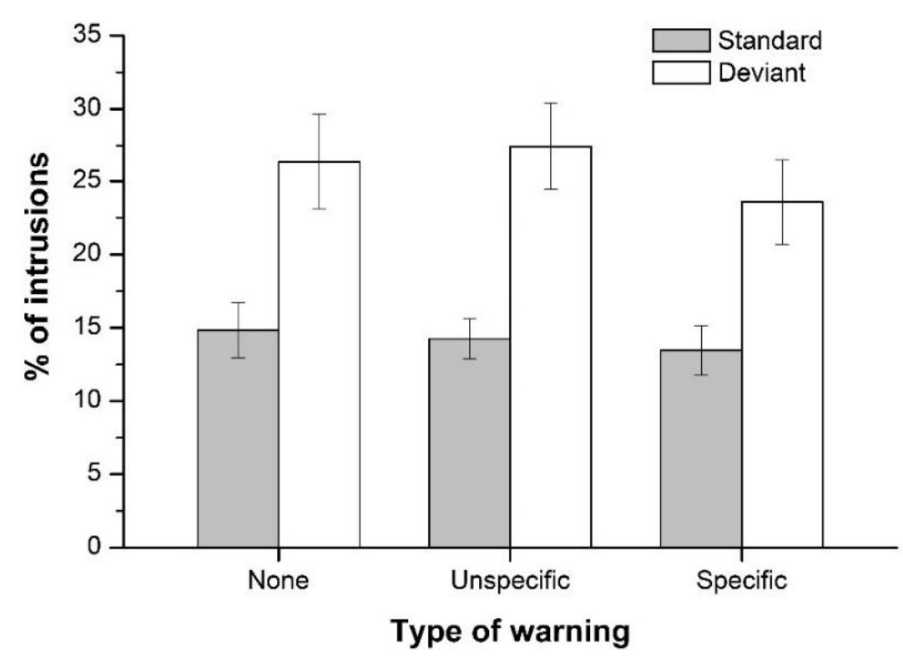

Figure 12. Mean percentage of intrusions of the missing item when absent from the trial (standard) and when presented as the auditory deviant (deviant) in the three warning conditions of Experiment 7. Error bars represent 95\% within-subject confidence intervals.

\section{Discussion}

The results of Experiment 7 are clear-cut: serial recall performance was impaired and intrusions of the missing item were more frequent in deviant relative to standard trials regardless of whether a pre-trial warning cue was provided or not. Whereas previous experiments showed that the mere notification that an unspecified change of category would occur next had no effect on distraction, the current experiment extended such a finding to the forewarning of the exact identity of the deviant item. Indeed, the disruptive effect of categorical deviants was found even when participants knew beforehand which (deviant) digit was going to be embedded in the irrelevant auditory sequence. This means that even distraction caused by a highly predictable categorical deviation cannot be resisted. Such results contrast with those of Röer and colleagues (2015), who found that comparable foreknowledge manipulations reduced distraction with 
sentential materials. The present pattern of results provides further evidence that the disruption of short-term memory by categorical deviations within TBI auditory sequences reflects the consequence of an uncontrollable process that is immune to top-down control. We suggest this process is the preattentive extraction of the meaning of the irrelevant items.

\section{Bayes Factor Meta-Analysis}

Bayesian techniques were used to determine the relative level of support for our theoretically derived categorical deviant hypothesis as well as our top-down manipulations hypothesis over the null hypothesis. Bayes factors provide a continuous measure of how probable the data are under our hypotheses compared to how probable the data are under the null hypothesis. Bayes factor calculations were undertaken using software described in Dienes (2008, $2011,2014)$. This assumes, as a default, a null hypothesis where the true population value is exactly zero. The Bayesian approach demands specificity about the hypothesis to be contrasted with the null. Although no prior research has unveiled a categorical deviation effect, we assumed that the effect would be similar in magnitude to the acoustical version of the effect. Therefore, our assumption was that a categorical deviation effect would vary in size between zero and the upper limit set by the acoustical deviation effect. We based our prediction on a half-normal distribution wherein predicting smaller effect sizes—such as the deviation effects—is more likely than large effect sizes. Here, the estimate of the standard deviation of the p(population value|theory) was computed as an average of the mean difference between deviant and nodeviant conditions (7.12; $S E=1.62)$ from Körner, Röer, Buchner, \& Bell (2017; Experiment 3), Sörqvist (2010, Experiments 1 and 2), and Vachon and colleagues (2017, Experiments 1A and 1B) and mean of $\mathrm{p}$ (population value|theory) was set at 0 . Data from the current ten experiments $(1 \mathrm{~A}, 1 \mathrm{~B}, 2 \mathrm{~A}, 2 \mathrm{~B}, 3 \mathrm{~A}, 3 \mathrm{~B}, 4,5,6$ and 7$)$ were combined in a meta-analysis using the mean and standard deviation $(S D)$ from Experiment 1A as the prior mean and prior $S D$ and the mean from 
Experiment $1 \mathrm{~B}$ as the likelihood mean and likelihood's $S D$ in order to calculate posterior mean and posterior $S D$. Once obtained this was then entered as the new prior mean and $S D$ and the mean and $S D$ from Experiment 2 used as the likelihood's mean and $S D$. Once all ten studies had been included in this stepwise procedure (Dienes, 2008) a final Bayes Factor was computed to represent the combined data. The Bayes Factor was $\mathrm{B}_{\mathrm{H}(0,7.12)}=3.036853124598074 \times 10^{41}$. Therefore, the results indicate extreme evidence for the alternative, categorical deviation hypothesis, over the null hypothesis (see Jeffreys, 1961).

We applied the same technique separately for the task-engagement manipulation and the forewarning manipulation. With regard to task engagement, the estimate of the standard deviation of the $\mathrm{p}$ (population value|theory) was computed as the mean difference in the size of the deviation effect (i.e. the mean difference between deviant and no-deviant conditions) between the low- and high-difficulty conditions $(9.34 ; S E=2.35)$ found in Experiment 1 of Hughes and colleagues (2013). Data from Experiments 4 and 6 were combined in a metaanalysis. The data indicated there was moderate evidence for the null hypothesis, $\mathrm{B}_{\mathrm{H}(0,9.34)}=$ 0.19. Concerning the effect of foreknowledge, the estimate of the standard deviation of the $\mathrm{p}$ (population value|theory) was computed as the mean difference in the size of the deviation effect between the no-warning and warning conditions $(8.42 ; S E=3.15)$ found in the lowdifficulty condition of Experiment 2 of Hughes and colleagues (2013). Data from Experiments 4, 6 , and 7 were combined in a meta-analysis. The data indicated there was strong evidence for the null hypothesis, $\mathrm{B}_{\mathrm{H}(0,8.42)}=0.10$. Such results provide convincing evidence that the categorical deviation effect was not affected by top-down manipulations.

\section{General Discussion}

The purpose of the present study was to revisit the debate about the automaticity of semantic processing using a new approach: the automatic access to the meaning of task- 
irrelevant speech was tested through the assessment of its distractive power. More specifically, we aimed to examine whether the insertion of an unexpected category change within the content of a TBI sequence of neutral and non-significant spoken items could disrupt focal processing. We established that the presence of such a categorical deviation within the irrelevant auditory stream impaired the serial recall of visual items and that the deviant item tended to intrude into recall, indicating that the change in the content of the sound was detected. In seven experiments, we sought to determine whether such categorical deviance detection took place automatically by assessing the functional properties of this novel categorical deviation effect.

In the first part of the current paper, our findings revealed that the categorical deviation effect occurred with non-significant deviations (i.e., that carried no motivational value), meaning that distraction was induced by a (contextual) change in the content of the irrelevant sequence and not by the meaningfulness of the deviant stimulus. The effect was found with distractors that were either semantically impoverished (alphanumerical stimuli) or semantically rich (semantic category exemplars). This form of distraction was not contingent on either the relationship between the TBR and TBI material or the activation of semantic or verbal codes in the focal task, suggesting that categorical deviance detection was not primed by a particular configuration of the cognitive system designed to facilitate semantic processing. In addition, it was not influenced by the novelty of the deviant in that its probability compared to "standards" did not influence the disruption it produced. Finally, the effect manifest in the absence of acoustical and/or phonological discrepancies between the deviant and standard stimuli, suggesting that its origin is not acoustic.

In the second part of this article, we demonstrated that the categorical deviation effect was impervious to cognitive control. Indeed, the effect was not influenced by top-down manipulations. Increasing the level of engagement in the focal task—or concentration-by 
making the TBR items more difficult to perceptually discriminate failed to reduce the amplitude of the deviation effect or the amount of deviant intrusion. Similarly, deviant disruption and intrusion remained unaffected when participants were warned about the imminent occurrence of the categorical change in the irrelevant stream, even if such forewarning specified the identity of the deviant stimulus. This convergence of experimental and psychometric data indicate that the categorical deviation effect was triggered independently from the current task set, suggesting that the semantic content of the irrelevant speech was processed in a purely automatic fashion.

\section{Automaticity and Semantic Processing}

Following Moors and De Houwer's $(2006,2007)$ approach, we aimed to diagnose the automaticity of semantic processing by assessing whether this form of processing was intentional or unintentional, goal-dependent or goal-independent, and controllable or uncontrollable. For a process to qualify as unintentional, the process must be present in the absence of intention to activate the process. The methodology and task-settings we used to assess the disruptive impact of the post-categorical properties of irrelevant sound arguably satisfy the stringent criteria of ensuring that TBI information was processed unintentionally. Indeed, the key stimulation was presented outside the focus of attention, was completely task-irrelevant and participants were informed they would never be tested on its content. Therefore, there was little, if any, incentive for the participants to process the sound intentionally, with maybe the exceptions of Experiments 5-7 whereby the forewarning of an imminent categorical change may have promoted transient attentional diversion toward the incoming deviant out of curiosity. Moreover, the focal serial recall tasks used in the present series of experiments are characterized by the unrelenting, attentionally-demanding serial rehearsal process. And yet task performance was impaired by the presence of a semantic change in the TBI auditory stream in each of the seven experiments of the present study, indicating that the categorical deviation was systematically detected. Such 
detection must rely on the processing of the content of the auditory sequence, suggesting that semantic processing happened unintentionally.

Despite these controls, one could argue that detection of a categorical deviation ensued from attentional resources being allocated to irrelevant speech as the result of intermittent attentional slips (Lachter et al., 2004) or co-monitoring of the auditory sequence (Röer et al., 2017a). Ensuring that attention slippage never occurs is extremely difficult (e.g., Rivenez, Darwin, \& Guillaume, 2006), but the results of Experiments 4-7 strongly suggest that slippage played no role in the categorical deviation effect. On the one hand, the effect remained unaffected by making the TBR items more difficult to discriminate (see Experiments 4 and 6), a manipulation known to increase the level of engagement (or concentration) in the task. When people concentrate harder, their locus of attention becomes more steadfast (i.e., not as easily diverted by irrelevant information; see Sörqvist \& Marsh, 2015), hence reducing the propensity of attentional slips toward the irrelevant channel. If the semantic analysis of the TBI sequence was dependent upon such slips, the detection of a categorical deviation-and the ensuing distraction —-would have been less likely under high task engagement. However, the potency of the categorical deviation was not altered when task engagement increased, suggesting that attentional slippage plays no role in the phenomenon. On the other hand, providing participants with foreknowledge of the incoming deviant may promote (voluntary) slips of attention toward the irrelevant stream out of curiosity for that special distractor, and yet forewarning even the identity of the deviant item failed to increase the amount of disruption-including the number of intrusions—produced by that deviant (see Experiments 5-7). We are therefore confident that the irrelevant auditory sequence was never attended, other than when attention was captured by a deviant, and, hence, that the content of that sequence was analyzed and extracted unintentionally. 
In order to be considered goal-independent, a process must not depend on a goal for its occurrence, neither proximal nor remote. In the present series of experiments, the categorical deviation effect was task-invariant: it took place regardless of the process required by the focal task. The semantic processing of the TBI stream occurred even when the task-process per se was not a semantically-based one. That is, the key process underpinning performance in the serial recall task is an articulatory-based seriation process (Jones et al., 2004; Meade \& Fernandes, 2016) that is devoid of semantic processing. Yet, one could argue that the use of verbal items as TBR material (in Experiments 1, 2, 6 and 7) activated a remote goal that triggered verbal and/or semantic processing directly, circumventing the proximal goal of processing serial order. In such a case, the processing responsible for the detection of the categorical change would be considered goal-dependent. However, the categorical deviation effect was not conditional on the activation of mere verbal codes in the focal task as it was found with both verbal and non-verbal memoranda (see Experiments 3-5). Therefore, the degree to which the semantic content of TBI items is processed cannot be accounted for by some cross-modal priming initiated by the need to process verbal stimuli. According to Moors and De Houwer (2006), the features (un)intentional and goal-(in)dependent are intertwined: processes that are not contingent on a proximal goal constitute, by definition, a form of unintentional processes. Therefore, the evidence that the semantic processing responsible for the categorical deviation effect is goal-independent provides further support for its unintentional nature.

A process is said to be controllable when the goal (to engage in, to avoid, to alter or to interrupt the process) is a fundamental part of the sufficient set of conditions for the effect (occurrence, prevention, change, or interruption of the process). On the opposite, an uncontrollable process is one for which there is no such set. Therefore, whether there is a goal pertaining to the process or not, there should be no effect on an uncontrollable process. The 
results from Experiments 4 to 7 revealed that the amount of disruption produced by a categorical deviation seems impervious to top-down control factors such as the level of engagement in the task and foreknowledge about the imminent deviant. Indeed, these manipulations failed to alter the behavioral manifestation of the semantic deviation effect. Such immunity to cognitive control is consistent with the view that the semantic processing at the origin of the phenomenon is uncontrollable.

Moors and De Houwer (2006, 2007) also identified the feature autonomous, which corresponds to processes uncontrollable in terms of every possible processing goal. This implies that a completely autonomous process is not produced, altered, stopped, or avoided by a processing goal. The present study was not designed to directly assess whether semantic processing runs autonomously or not. However, the fact that the categorical deviation effect seems to be an ineluctable form of distraction (it was systematically found in the 21 conditions tested in the present study without being tempered) suggests that the semantic processes causing the effect can run autonomously. Again, there is considerable overlap with other features: autonomous processes can be viewed as a subclass of not only uncontrollable processes, but also of unintentional processes. Given the present findings provide evidence that the semantic processing of the irrelevant speech was unintentional and uncontrollable, one could therefore argue that it is also autonomous. More work is required however to ascertain the autonomous nature of semantic processing in the current paradigm.

The current work provided converging evidence that the processing behind the detection of the transient categorical change within the irrelevant auditory stream can be at the same time unintentional, goal-independent, uncontrollable, and possibly autonomous. Therefore, we can conclude unambiguously that semantic processing can be purely or fully automatic (cf. Posner \& Snyder, 1975; Schneider \& Shiffrin, 1977). Such a conclusion is in stark contrast with previous 
claims that semantic processing, as many other forms of automatic processes, is critically dependent on top-down factors such as attention, intention, and task sets (e.g., Kiefer, 2007; Kiefer \& Martens, 2010; Naccache et al., 2002; Neumann, 1984, 1990). This view that semantic processing is contingent on the precise configuration of the cognitive system stems mainly from the literature on semantic priming (see, e.g., Kiefer \& Martens, 2010). Inspired by the work of Bargh (1992), Moors and De Houwer (2006) posited that all automatic processes are dependent on a set of preconditions that may vary from one process to another and proposed that "the study of automaticity should be concerned with establishing the set of preconditions that must be in place for an autonomous process to occur" (p. 302). We consider that this set of preconditions might vary according to the methodological parameters used to assess the automatic nature of a process.

The masked semantic priming technique consists of measuring the facilitative effect elicited by an unconsciously perceived masked stimulus, the prime, on the response to a subsequently presented visible stimulus, the target. In order to obtain facilitation, the (irrelevant) prime and the target need to be related in some way. Consequently, the content of the subliminal prime is likely to match the configuration of the cognitive system set to process the target adequately (Ratcliff \& McKoon, 1988). Such (pre)conditions are therefore likely to render the processing of the prime more susceptible to the influence of the established task set(s). In the paradigm used in the present study, care was taken to ensure that the content of the irrelevant stimuli would not match task requirements. In addition, the prime is typically presented in the same location as the target, hence it occurs at the attended location. Presenting the prime in the focus of attention is likely to increase its sensitivity to attentional effects even though it is not consciously perceived (e.g., Kellenbach \& Michie, 1996). With the irrelevant speech technique employed here, the semantic material was presented in a different sensory modality than the 
task-relevant information and participants were explicitly told to ignore it. It thus appears that the conflicting conclusions with regard to the automatic nature of semantic processing between the current study and work on semantic priming most likely originate from key differences in the methods employed. This observation is in line with Bargh's (1992) recommendation: "researchers should pay careful attention to aspects of their paradigms that might contribute to the production of an automatic effect, and attempt to remove these preconditions in order to gain an accurate picture of the necessary conditions for the effect" (p. 193).

\section{Implications for Auditory Distraction}

Eimer and colleagues (1996) made a distinction between specific and aspecific forms of distraction. Distraction is specific when the power of a stimulus to disrupt ongoing processing derives specifically from the special meaning of the stimulus itself (e.g., motivationally-relevant stimuli). In stark contrast, the categorical deviation effect cannot be considered as a specific form of distraction since there is nothing about the deviant per se (e.g., a letter or a digit) that endows it with attention-grabbing power. In fact, the distractive potency of the categorical deviant is entirely dependent on the fact that it is discrepant from the semantic context provided by previous distractors, which is more consistent with the definition of aspecific distraction.

Semantic distraction effects have been reported in the context of aspecific attentional capture. Indeed, when a sound captures attention by virtue of the fact that it deviated from the acoustical invariance characterizing the irrelevant stimulation, its content can sometimes produce further disruption. For example, the categorization of a visual target was slowed down by a deviant auditory stimulus that conveyed semantic information incongruent with the meaning of that target compared to congruent semantic content (e.g., Parmentier, 2008; Parmentier \& Kefauver, 2015; Parmentier et al., 2014). Given that such semantic effects are exclusive to attention-capturing sounds, researchers assumed that the semantic analysis of irrelevant auditory 
material could take place only once attention had been involuntary diverted toward the sound (Escera et al., 2003; Parmentier, 2008; Parmentier \& Kefauver, 2015; Roye et al., 2007). One exception is the study of Marsh, Röer, Bell, and Buchner (2014), who reported that the disruption of visual serial recall was amplified when an acoustical deviant (the repetition of a spoken item embedded within an otherwise changing sequence of items) violated a well-known canonical sequence (e.g., $12345 \underline{5} 678$ ). The authors concluded that such findings provide evidence that the post-categorical content of the auditory sequence must have been processed before the acoustical deviant occurred. However, a sequence of digits presented in canonical order is an overlearned sequence. This means that the acoustical transitions from one item to the next one are more easily predictable in a canonical sequence than in a random sequence. Hence, any deviation from this highly expected acoustical pattern should trigger a larger attentional response. It is therefore impossible to determine with certainty whether the enlarged distraction found by Marsh, Röer and colleagues with canonical sequences is of semantic or acoustical nature. The present findings unambiguously indicate that the categorical deviation effect is purely semantic because distraction was observed with stimuli that were not acoustically discrepant from previously encountered distractor sounds. There was nothing in the auditory sequences used in the present study, apart from the rare and unexpected categorical change, that could have triggered an orienting response. Moreover, for a change of category to be detected, a categorical context must be established in the first place, which requires the preceding auditory stimuli of the sequence to undergo semantic analysis. The semantic processing of the irrelevant sound that leads to the categorical deviation effect cannot, therefore, be ascribed to attention being captured and (re)oriented toward the auditory channel. Instead, we claim that the effect took place because the content of the TBI sequence was processed preattentively (see also Röer et al., 2017b, for a similar conclusion based on a semantic priming effect from irrelevant speech). 
Taking for granted the notion that the post-categorical properties of the irrelevant sound material are processed preattentively, at what level does such processing operate? Are sequences of words semantically analyzed to extract sentential meaning or a representation of their shared category membership? Or is the meaning of individual words processed without their integration with other words? And what consequence does this semantic processing have for the focal task processing? The current data clearly support the notion that the semantic relationship between consecutive words within the unattended speech is processed, otherwise the categorical deviation would not be processed as such and therefore fail to disrupt serial recall performance. This conclusion contradicts earlier suggestion that unattended information undergoes semantic analysis but only at the level of individual word meanings (Marsh, Perham, Sörqvist, \& Jones, 2014; Underwood \& Everatt, 1996). For example, in their review Underwood and Everatt (1996) concluded that there is little evidence that unattended sequences of words gain the integration necessary for recognition of their underlying meaning which requires a propositional analysis of an irrelevant sentence thought to require focal attention. More specifically, they argue that listeners do not appear to recognize the deep structure of an unattended sentence or recognize the common category of words in an unattended list. Similarly, Marsh, Perham and colleagues (2014) concluded that the disruption meaningful irrelevant sound produces to semantic categoryclustering was due to semantic processing of individual lexical items, rather than supra-lexical, semantic processing. This conclusion was shaped by the finding that the order of approximation to English of irrelevant narrative had no effect on the degree to which it disrupted semanticcategorization: Natural English narrative (first-order approximation) produced no more disruption than a high-order approximation to English (whereby every sixth word of a text is selected and randomly reinserted into the vacant positions) and a low-order approximation (whereby this procedure is performed with every second word). 
A widely accepted explanation of the acoustical deviation effect is that an acousticallydeviating sound captures attention to the degree that its acoustical properties violate expectations of an impending sound, or those of an immediately preceding experience (e.g., Cowan, 1995; Schröger, 1997). An automatic deviance-detection mechanism requires that a mental description of incoming auditory stimulation has been preattentively fashioned. Such a 'neural model' (cf. Sokolov, 1963) is described as an abstract forward (or predictive) mnemonic representation derived from the acoustical regularities embodied in the unfolding auditory stimulation (e.g., Bendixen et al., 2007; Hughes et al., 2007; Vachon et al., 2012; Winkler et al., 2009). Accordingly, it has been assumed that only acoustical—i.e. pre-categorical—information can be extracted and encoded into the neural model (cf. Cowan, 1995). The fact that a categorical deviation within the irrelevant auditory stream can disrupt serial recall in the same way as an acoustical deviation suggests that post-categorical features can also be represented in the neural model (see also Marsh, Röer et al., 2014; Röer, Bell, Körner, \& Buchner, 2019). In fact, the current results are consistent with the notion that automatic semantic processing is involved in the rapid fabrication of a neural model based on post-categorical details of the TBI auditory sequence. Since the presentation of only five category exemplars in Experiment 2B was sufficient for a categorical deviant to be detected and, in turn to capture attention, the establishment of a post-categorical neural model appears to be rather fast.

What type of semantic information would be included in the neural model? As discussed earlier and based on the present results, we assume that what is extracted and encoded into the neural modal is not an aggregate (cf. Hughes et al., 2005, 2007) of the semantic features of previously encountered spoken items (e.g., the meaning of each individual items), but rather the semantic relationship transcending the items composing the unfolding auditory sequence such as category membership. Recently, Röer and colleagues (2019) showed that visual-verbal serial 
recall is more disrupted by TBI auditory sentences with unexpected endings than sentences with expected endings. The authors interpreted such a 'semantic mismatch effect' in terms of attentional capture. These findings suggest that sentential meaning could also be preattentively represented in the neural model. Yet, recent evidence that TBI English single sentences produce more disruption than TBI Swedish single sentences for English monolinguals (Marsh, Kershaw, Vachon, \& Hughes, 2019) suggests that some sort of attentional diversion toward the irrelevant speech must occur prior to the sentence ending. Therefore, the jury is still out as to whether sentence ending violations produce capture when attention is focussed elsewhere and, accordingly, whether sentential meaning is processed preattentively. As for acoustical information (cf. Schröger, 1997), we postulate that an implicit process compares the semantic representation of the current sound to the post-categorical predictions generated by the neural model. Hence, a categorical deviant tends to trigger a 'call for attention' when its content mismatches the implicit (semantic) expectancies derived from the neural model. When this call is answered, the attentional focus is diverted away from the focal task, leading to performance disruption, and momentarily displaced onto the deviant, fostering a deeper analysis of the distractor (cf. Escera et al., 2003; Parmentier, 2008) that makes it more likely to be perceived as a task-relevant stimulus and, therefore, to be erroneously recalled.

The fact that not only categorical deviants disrupted visual serial recall but also tended to intrude into recall is consistent with the idea that the form of distraction highlighted here is underpinned by attentional capture. However, the apparent insensitivity of the phenomenon to top-down control and its independence from the acoustical deviation effect cast some doubt about the attention-capture hypothesis of the categorical deviation effect. Indeed, attentional capture is typically viewed as a form of distraction that is amenable to top-down cognitive control (e.g., Awh, Belopolsky, \& Theeuwes, 2012; De Jong, Berendsen, \& Cools, 1999). 
Besides, there is evidence that the attentional response to acoustical deviations is influenced by such control: promoting focal-task engagement and providing foreknowledge of an imminent deviation have been shown to temper the distractive impact of acoustical deviants (e.g., Horváth \& Bendixen, 2012; Hughes et al., 2013; Shelton et al., 2009; Sussman et al., 2003). The present findings indicate this is not the case for categorical deviants, suggesting that the acoustical and categorical deviation effects are not completely functionally equivalent and may, therefore, be produced by distinct mechanisms. For instance, it is assumed that when the occurrence of an acoustical deviant can be anticipated by virtue of a pre-trial forewarning, it can be voluntarily incorporated into the neural model (Bendixen et al., 2007; Hughes et al., 2013; Winkler et al., 2009), so that the deviation would no longer be considered as a violation of expectancies derived from the neural model. The finding that categorical deviation disruption and intrusion endured even when participants were provided beforehand with the identity of the deviant item appears inconsistent with an interpretation of the categorical deviation effect in terms of an orienting response elicited by the violation of a (post-categorical) predictive model. Further examination of the functional properties of the categorical deviation effect is required to pinpoint with more precision the mechanisms underlying this form of auditory distraction.

\section{Conclusion}

The present study highlighted a novel auditory distraction phenomenon whereby a rare and unexpected change of category within an irrelevant stream of spoken items disrupted visual serial recall. Overall, our findings revealed that this categorical deviation effect is not contingent on the activated task set and appears resistant to top-down control. By suggesting not only that the semantic content of the irrelevant sound can be extracted unintentionally, independently from activated goals and in an uncontrollable fashion, but also that such semantic activation seems ineluctable, these findings put a new light on the automatic nature of semantic processing. The 
distraction paradigm exploited in the current research offers a new tool for studying automatic processes as it provides a way to assess processing of information that is never attended and that is neither related nor relevant to the configuration of the cognitive system. It is in such an experimental context that the present study demonstrated, at odds with recent research, that semantic processing can indeed be fully automatic. 


\section{References}

Anderson, B. A. (2013). A value-driven mechanism of attentional selection. Journal of Vision, 13(3), 7. doi:10.1167/13.3.7

Ansorge, U., \& Horstmann, G. (2007). Preemptive control of attentional capture by colour: Evidence from trial-by-trial analyses and orderings of onsets of capture effects in reaction time distributions. Quarterly Journal of Experimental Psychology, 60, 952-975. doi:10.1080/17470210600822795

Ansorge, U., Kunde, W., \& Kiefer, M. (2014). Unconscious vision and executive control: How unconscious processing and conscious action control interact. Consciousness and Cognition, 27, 268-287. doi:10.1016/j.concog.2014.05.009

Awh, E., Belopolsky, A. V., \& Theeuwes, J. (2012). Top-down versus bottom-up attentional control: a failed theoretical dichotomy. Trends in Cognitive Sciences, 16, 437-443. doi:10.1016/j.tics.2012.06.010

Ball, L. J., Threadgold, E., Solowiej, A., \& Marsh, J. E. (2018). Can intrinsic and extrinsic metacognitive cues shield against distraction in problem solving? Journal of Cognition, 1, 15. doi: $10.5334 /$ joc. 9

Bargh, J. A. (1989). Conditional automaticity: Varieties of automatic influence in social perception and cognition. In J. S. Uleman \& J. A. Bargh (Eds.), Unintended thought (pp. 3-51). New York: Guilford Press.

Bargh, J. A. (1992). The ecology of automaticity: Toward establishing the conditions needed to produce automatic processing effects. American Journal of Psychology, 105, 181-199. doi: $10.2307 / 1423027$ 
Beaman, C. P. (2004). The irrelevant sound phenomenon revisited: What role for working memory capacity? Journal of Experimental Psychology: Learning, Memory, and Cognition, 30, 1106-1118. doi:10.1037/0278-7393.30.5.1106

Beaman, C. P., \& Jones, D. M. (1997). The role of serial order in the irrelevant speech effect: Tests of the changing state hypothesis. Journal of Experimental Psychology: Learning, Memory, and Cognition, 23, 459-471. doi:10.1037//0278-7393.23.2.459

Bell, R., Röer, J. P., Marsh, J. E., Storch, D., \& Buchner, A. (2017). The effect of cognitive control on different types of auditory distraction: A preregistered study. Experimental Psychology, 64, 359-368. doi:10.1027/1618-3169/a000372

Bendixen, A., Roeber, U., \& Schröger, E. (2007). Regularity extraction and application in dynamic auditory stimulus sequences. Journal of Cognitive Neuroscience, 19, 1664-1677. doi:10.1162/jocn.2007.19.10.1664

Besner, D., Stolz, J. A., \& Boutilier, C. (1997). The Stroop effect and the myth of automaticity. Psychonomic Bulletin \& Review, 4, 221-225. doi:10.3758/bf03209396

Boersma, P., \& Weenink, D. (2019). Praat: doing phonetics by computer [Computer program]. Version 6.0.56, retrieved 10 July 2019 from http://www.praat.org/

Botvinick, M. M., Braver, T. S., Barch, D. M., Carter, C. S., \& Cohen, J. D. (2001). Conflict monitoring and cognitive control. Psychological Review, 108, 624-652. doi:10.1037//0033-295X.I08.3.624

Braver, T. S. (2012). The variable nature of cognitive control: A dual mechanisms framework. Trends in Cognitive Sciences, 16, 106-113. doi:10.1016/j.tics.2011.12.010

Bregman, A. S. (1990). Auditory scene analysis: The perceptual organization of sound. Cambridge, MA: The MIT Press. 
Buchner, A., Mehl, B., Rothermund, K., \& Wentura, D. (2006). Artificially induced valence of distractor words increases the effects of irrelevant speech on serial recall. Memory \& Cognition, 34, 1055-1062. doi:10.3758/bf03193252

Buchner, A., Rothermund, K., Wentura, D., \& Mehl, B. (2004). Valence of distractor words increases the effects of irrelevant speech on serial recall. Memory \& Cognition, 32, 722731. doi:10.3758/bf03195862

Buetti, S., \& Lleras, A. (2016). Distractibility is a function of engagement, not task difficulty: Evidence from a new oculomotor capture paradigm. Journal of Experimental Psychology: General, 145, 1382-1405. doi:10.1037/xge0000213

Buschke, H. (1963). Relative retention in immediate memory determined by the missing scan method. Nature, 200, 1129-1130. doi:10.1038/2001129b0

Conway, A. R., Cowan, N., \& Bunting, M. F. (2001). The cocktail party phenomenon revisited: The importance of working memory capacity. Psychonomic Bulletin \& Review, 8, 331335. doi:10.3758/bf03196169

Cousineau, D. (2005). Confidence intervals in within-subject designs: A simpler solution to Loftus and Masson's method. Tutorials in Quantitative Methods for Psychology, 1, 4245. doi:10.20982/tqmp.01.1.p042

Cowan, N. (1995). Attention and memory: An integrated framework. Oxford: Oxford University Press. doi:10.1093/acprof:oso/9780195119107.001.0001

Dalton, P., \& Lavie, N. (2004). Auditory attentional capture: Effects of singleton distractor sounds. Journal of Experimental Psychology: Human Perception and Performance, 30, 180-193. doi:10.1037/0096-1523.30.1.180

Dalton, P., \& Lavie, N. (2007). Overriding auditory attentional capture. Perception \& Psychophysics, 69, 162-171. doi:10.3758/bf03193739 
De Jong, R., Berendsen, E., \& Cools, R. (1999). Goal neglect and inhibitory limitations:

Dissociable causes of interference effects in conflict situations. Acta Psychologica, 101, 379-394. doi:10.1016/S0001-6918(99)00012-8

Dehaene, S., Naccache, L., Le Clec'H, G., Koechlin, E., Mueller, M., Dehaene-Lambertz, G., van de Moortele, P. F., \& Le Bihan, D. (1998). Imaging unconscious semantic priming. Nature, 395, 597-600. doi:10.1038/26967

Dienes, Z. (2008). Understanding psychology as a science: An introduction to scientific and statistical inference. Basingstoke: Palgrave Macmillan.

Dienes, Z. (2011). Bayesian versus orthodox statistics: Which side are you on? Perspective in Psychological Science, 6, 274-290. doi:10.1177/1745691611406920

Dienes, Z. (2014). Using Bayes to get the most out of non-significant results. Frontiers in Psycholology, 5, 781. doi:10.3389/fpsyg.2014.00781

Eggemeier, F. T., Crabtree, M. S., \& LaPointe, P. A. (1983). The effect of delayed report on subjective ratings of mental workload. Proceedings of the Human Factors and Ergonomics Society Annual Meeting, 27(2), 139-143. doi:10.1177/154193128302700205

Eimer, M., Nattkemper, D., Schröger, E., \& Prinz, W. (1996). Involuntary attention. In O. Neumann \& A. F. Sanders (Eds.), Handbook of perception and action (Vol. 3, pp. 155184). San Diego, CA: Academic Press. doi:10.1016/S1874-5822(96)80022-3

Escera, C., Alho, K., Winkler, I., \& Näätänen, R. (1998). Neural mechanisms of involuntary attention to acoustic novelty and change. Journal of Cognitive Neuroscience, 10, 590 604. doi:10.1162/089892998562997

Escera, C., Yago, E., Corral, M. J., Corbera, S., \& Nuñez, M. I. (2003). Attention capture by auditory significant stimuli: semantic analysis follows attention switching. European Journal of Neuroscience, 18, 2408-2412. doi:10.1046/j.1460-9568.2003.02937.x 
Formby, D. (1967). Maternal recognition of infant's cry. Developmental Medicine \& Child Neurology, 9, 293-298. doi:10.1111/j.1469-8749.1967.tb02271.x

Guérard, K., \& Tremblay, S. (2008). Revisiting evidence for modularity and functional equivalence across verbal and spatial domains in memory. Journal of Experimental Psychology: Learning, Memory, and Cognition, 34, 556-569. doi:10.1037/02787393.34.3.556

Hackley, S. A. (1993). An evaluation of the automaticity of sensory processing using eventrelated potentials and brain-stem reflexes. Psychophysiology, 30, 415-428. doi:10.1111/j.1469-8986.1993.tb02065.x

Halin, N. (2016). Distracted while reading? Changing to a hard-to-read font shields against the effects of environmental noise and speech on text memory. Frontiers in Psychology, 7, 1196. doi:10.3389/fpsyg.2016.01196

Halin, N., Marsh, J. E., Haga, A., Holmgren, M., \& Sörqvist, P. (2014). Effects of speech on proofreading: Can task-engagement manipulations shield against distraction? Journal of Experimental Psychology: Applied, 20, 69-80. doi:10.1037/xap0000002

Halin, N., Marsh, J. E., \& Sörqvist, P. (2015). Central load reduces peripheral processing: Evidence from incidental memory of background speech. Scandinavian Journal of Psychology, 56, 607-612. doi:10.1111/sjop.12246

Hanczakowski, M., Beaman, C. P., \& Jones, D. M. (2017). When distraction benefits memory through semantic similarity. Journal of Memory and Language, 94, 61-74. doi:10.1016/j.jml.2016.11.005

Holeckova, I., Fischer, C., Giard, M.H., Delpuech, C. \& Morlet, D. (2006). Brain responses to a subject's own name uttered by a familiar voice. Brain Research, 1082, 142-152. doi:10.1016/j.brainres.2006.01.089 
Holender, D. (1986). Semantic activation without conscious identification in dichotic listening, parafoveal vision, and visual masking: A survey and appraisal. Behavioral and Brain Sciences, 9, 1-66. doi:10.1017/s0140525x00021269

Horváth, J., \& Bendixen, A. (2012). Preventing distraction by probabilistic cueing. International Journal of Psychophysiology, 83, 342-347. doi: 10.1016/j.ijpsycho.2011.11.019

Hughes, R. W. (2014). Auditory distraction: A duplex-mechanism account. PsyCH Journal, 3, 30-41. doi:10.1002/pchj.44

Hughes, R. W., Hurlstone, M. J., Marsh, J. E., Vachon, F., \& Jones, D. M. (2013). Cognitive control of auditory distraction: Impact of task difficulty, foreknowledge, and working memory capacity supports duplex-mechanism account. Journal of Experimental Psychology: Human Perception and Performance, 39, 539-553. doi:10.1037/a0029064

Hughes, R. W., \& Jones, D. M. (2003). Indispensable benefits and unavoidable costs of unattended sound for cognitive functioning. Noise \& Health, 6, 63-76.

Hughes, R. W., Vachon, F., \& Jones, D. M. (2005). Auditory attentional capture during serial recall: Violations at encoding of an algorithm-based neural model? Journal of Experimental Psychology: Learning, Memory, and Cognition, 31, 736-749. doi:10.1037/0278-7393.31.4.736

Hughes, R. W., Vachon, F., \& Jones, D. M. (2007). Disruption of short-term memory by changing and deviant sounds: Support for a duplex-mechanism account of auditory distraction. Journal of Experimental Psychology: Learning, Memory, and Cognition, 33, 1050-1061. doi:10.1037/0278-7393.33.6.1050

Jeffreys, H. (1961). Theory of probability ( $3^{\text {rd }}$ ed.) Oxford: Oxford University Press.

Johnson, M. K., Hashtroudi, S., \& Lindsay, D. S. (1993). Source monitoring. Psychological Bulletin, 114, 3-28. doi:10.1037//0033-2909.114.1.3 
Jones, D. M. (1999). The cognitive psychology of auditory distraction: The 1997 BPS Broadbent Lecture. British Journal of Psychology, 90, 167-187. doi:10.1348/000712699161314

Jones, D., Farrand, P., Stuart, G., \& Morris, N. (1995). Functional equivalence of verbal and spatial information in serial short-term memory. Journal of Experimental Psychology: Learning, Memory, and Cognition, 21, 1008-1018. doi:10.1037/0278-7393.21.4.1008

Jones, D. M., Hughes, R. W., \& Macken, W. J. (2010). Auditory distraction and serial memory: The avoidable and the ineluctable. Noise \& Health, 12, 201-209. doi:10.4103/14631741.70497

Jones, D. M., Macken, W. J., \& Nicholls, A. P. (2004). The phonological store of working memory: Is it phonological and is it a store? Journal of Experimental Psychology: Learning, Memory, and Cognition, 30, 656-674. doi:10.1037/0278-7393.30.3.656

Jones, D., Madden, C., \& Miles, C. (1992). Privileged access by irrelevant speech to short-term memory: The role of changing state. Quarterly Journal of Experimental Psychology, 44A, 645-669. doi:10.1080/14640749208401304

Jones, D. M., \& Tremblay, S. (2000). Interference in memory by process or content? A reply to Neath (2000). Psychonomic Bulletin \& Review, 7, 550-558. doi:10.3758/bf03214370

Kahneman, D., \& Treisman, A. (1984). Changing views of attention and automaticity. In R. Parasuraman \& D. R. Davies (Eds.), Varieties of attention (pp. 29-61), Orlando, FL: Academic Press.

Keil, A., Bradley, M. M., Junghöfer, M., Russmann, T., Lowenthal, W., \& Lang, P. J. (2007). Cross-modal attention capture by affective stimuli: Evidence from event-related potentials. Cognitive, Affective, \& Behavioral Neuroscience, 7, 18-24. doi:10.3758/CABN.7.1.18 
Kellenbach, M. L., \& Michie, P. T. (1996). Modulation of event-related potentials by semantic priming: Effects of color-cued selective attention. Journal of Cognitive Neuroscience, 8 , 155-173. doi:10.1162/jocn.1996.8.2.155

Kiefer, M. (2002). The N400 is modulated by unconsciously perceived masked words: Further evidence for an automatic spreading activation account of N400 priming effects. Cognitive Brain Research, 13, 27-39. doi:10.1016/S0926-6410(01)00085-4

Kiefer, M. (2007). Top-down modulation of unconscious 'automatic' processes: A gating framework. Advances in Cognitive Psychology, 3, 289-306. doi:10.2478/v10053-008$0032-2$

Kiefer, M., \& Martens, U. (2010). Attentional sensitization of unconscious cognition: Task sets modulate subsequent masked semantic priming. Journal of Experimental Psychology: General, 139, 464-489. doi:10.1037/a0019561

Körner, U., Röer, J. P., Buchner, A., \& Bell, R. (2017). Working memory capacity is equally unrelated to auditory distraction by changing-state and deviant sounds. Journal of Memory and Language, 96, 122-137. doi:10.1016/j.jml.2017.05.005

Kreitz, C., Schnuerch, R., Furley, P. A., Gibbons, H., \& Memmert, D. (2015). Does semantic preactivation reduce inattentional blindness? Attention, Perception, \& Psychophysics, 77, 759-767. doi:10.3758/s13414-014-0819-8

Kunde, W., Kiesel, A., \& Hoffmann, J. (2003). Conscious control over the content of unconscious cognition. Cognition, 88, 223-242. doi:10.1016/s0010-0277(03)00023-4

Lachter, J., Forster, K. I., \& Ruthruff, E. (2004). Forty-five years after Broadbent (1958): Still no identification without attention. Psychological Review, 111, 880-913. doi:10.1037/0033295X.111.4.880 
Lange, E. B. (2005). Disruption of attention by irrelevant stimuli in serial recall. Journal of Memory and Language, 53, 513-531. doi:10.1016/j.jml.2005.07.002

Logan, G. D. (1988). Toward an instance theory of automatization. Psychological Review, 95, 492-527. doi:10.1037/0033-295X.95.4.492

Logan, G. D. (1989). Automaticity and cognitive control. In J. S. Uleman \& J. A. Bargh (Eds.), Unintended thought (pp. 52-74). New York: Guilford Press.

Logan, G. D., \& Schulkind, M. D. (2000). Parallel memory retrieval in dual-task situations: I. Semantic memory. Journal of Experimental Psychology: Human Perception and Performance, 26, 1072-1090. doi:10.1037//0096-1523.26.3.1072

Luck, S. J., Vogel, E. K., \& Shapiro, K. L. (1996). Word meanings can be accessed but not reported during the attentional blink. Nature, 383, 616-618. doi:10.1038/383616a0

Macken, W. J., Tremblay, S., Houghton, R. J., Nicholls, A. P., \& Jones, D. M. (2003). Does auditory streaming require attention? Evidence from attentional selectivity in short-term memory. Journal of Experimental Psychology: Human Perception and Performance, 29, 43-51. doi:10.1037/0096-1523.29.1.43

MacLeod, C. M. (1991). Half a century of research on the Stroop effect: An integrative review. Psychological Bulletin, 109, 163-203. doi:10.1037/0033-2909.109.2.163

Maki, W. S., Frigen, K., \& Paulson, K. (1997). Associative priming by targets and distractors during rapid serial visual presentation: Does word meaning survive the attentional blink? Journal of Experimental Psychology: Human Perception and Performance, 23, 1014-1034. doi:10.1037/0096-1523.23.4.1014

Marcel, A. J. (1983). Conscious and unconscious perception: An approach to the relations between phenomenal experience and perceptual processes. Cognitive Psychology, 15, 238-300. doi:10.1016/0010-0285(83)90010-5 
Marsh, J. E., \& Campbell, T. A. (2016). Processing complex sounds passing through the rostral brainstem: The new early filter model. Frontiers in Neuroscience, 10, 136. doi:10.3389/fnins.2016.00136

Marsh, J. E., Hughes, R. W., \& Jones, D. M. (2008). Auditory distraction in semantic memory: A process-based approach. Journal of Memory and Language, 58, 682-700. doi:10.1016/j.jml.2007.05.002

Marsh, J. E., Hughes, R. W., \& Jones, D. M. (2009). Interference by process, not content, determines semantic auditory distraction. Cognition, 110, 23-38. doi:10.1016/j.cognition.2008.08.003

Marsh, J. E., \& Jones, D. M. (2010). Cross-modal distraction by background speech: What role for meaning? Noise \& Health, 12, 210-216. doi:10.4103/1463-1741.70499

Marsh, J. E., Kershaw, M. B. A., Vachon, F., \& Hughes, R. W. (2019, in preparation). More than complex "changing-state": Meaningful single sentences produce attentional capture.

Marsh, J. E., Ljung, R., Jahncke, H., MacCutcheon, D., Ball, L. J., Pausch, F., \& Vachon, F. (2018). Why are background telephone conversations distracting? Journal of Experimental Psychology: Applied, 24, 222-235. doi:10.1037/xap0000170.

Marsh, J. E., Perham, N., Sörqvist, P., \& Jones, D. M. (2014). Boundaries of semantic distraction: Dominance and lexicality act at retrieval. Memory \& Cognition, 42, $1285-$ 1301. doi:10.3758/s13421-014-0438-6

Marsh, J. E., Röer, J. P., Bell, R., \& Buchner, A. (2014). Predictability and distraction: Does the neural model represent postcategorical features? PsyCh Journal, 3, 58-71. doi:10.1002/pchj.50

Marsh, J. E., Sörqvist, P., Hodgetts, H. M., Beaman, C. P., \& Jones, D. M. (2015). Distraction control processes in free recall: Benefits and costs to performance. Journal of 
Experimental Psychology: Learning, Memory, and Cognition, 41, 118-133. doi:10.1037/a0037779

Marsh, J. E., Sörqvist, P., \& Hughes, R. W. (2015). Dynamic cognitive control of irrelevant sound: Increased task engagement attenuates semantic auditory distraction. Journal of Experimental Psychology: Human Perception and Performance, 41, 1462-1474. doi:10.1037/xhp0000060

Marsh, J. E., Vachon, F., \& Jones, D. M. (2008). When does between-sequence phonological similarity promote irrelevant sound disruption? Journal of Experimental Psychology: Learning, Memory, and Cognition, 34, 243-248. doi:10.1037/0278-7393.34.1.243

Marsh, J. E., Vachon, F., \& Sörqvist, P. (2017). Increased distractibility in schizotypy: Independent of individual differences in working memory capacity? Quarterly Journal of Experimental Psychology, 70, 565-578. doi:10.1080/17470218.2016.1172094

Marsh, J. E., Yang, J., Qualter, P., Richardson, C., Perham, N., Vachon, F., \& Hughes, R. W. (2018). Postcategorical auditory distraction in serial short-term memory: Insights from increased task load and task type. Journal of Experimental Psychology: Learning, Memory, and Cognition, 44, 882-897. doi:10.1037/xlm0000492

Masson, M. E. J. (2011). A tutorial on a practical Bayesian alternative to null-hypothesis significance testing. Behavior Research Methods, 43, 679-690. doi:10.3758/s13428-010$0049-5$

Maxfield, L. (1997). Attention and semantic priming: A review of prime task effects. Consciousness and Cognition, 6, 204-218. doi:10.1006/ccog.1997.0311

Meade, M. E., \& Fernandes, M. A. (2016). The role of semantically related distractors during encoding and retrieval of words in long-term memory. Memory, 24, 801-811. doi:10.1080/09658211.2015.1053491 
Moors, A., \& De Houwer, J. (2006). Automaticity: A theoretical and conceptual analysis. Psychological Bulletin, 132, 297-326. doi:10.1037/0033-2909.132.2.297

Moors, A., \& De Houwer, J. (2007). What is automaticity? An analysis of its component features and their interrelations. In J. A. Bargh (Ed.), Automatic processes in social thinking and behavior. Hove, England: Psychology Press

Moray, N. (1959). Attention in dichotic listening: Affective cues and the influence of instructions. Quarterly Journal of Experimental Psychology, 11, 56-60. doi:10.1080/17470215908416289

Morey, C. C., \& Miron, M. D. (2016). Spatial sequences, but not verbal sequences, are vulnerable to general interference during retention in working memory. Journal of Experimental Psychology: Learning, Memory, and Cognition, 42, 1907-1918. doi:10.1037/xlm0000280

Morey, R. D. (2008). Confidence Intervals from Normalized Data: A correction to Cousineau (2005). Tutorials in Quantitative Methods for Psychology, 4, 61-64. doi:10.20982/tqmp.04.2.p061

Muller-Gass, A., Macdonald, M., Schröger, E., Sculthorpe, L., \& Campbell, K. (2007). Evidence for the auditory P3a reflecting an automatic process: Elicitation during highly-focused continuous visual attention. Brain Research, 1170, 71-78. doi:10.1016/j.brainres.2007.07.023

Murdock, B. B., Jr. (1993). TODAM2: A model for the storage and retrieval of item, associative, and serial-order information. Psychological Review, 100, 183-203. doi:10.1037//0033$295 x .100 .2 .183$

Naccache, L., Blandin, E., \& Dehaene, S. (2002). Unconscious masked priming depends on temporal attention. Psychological Science, 13, 416-424. doi:10.1111/1467-9280.00474 
Näätänen, R. (1990). The role of attention in auditory information processing as revealed by event-related and other brain measures of cognitive function. Behavioral and Brain Sciences, 13, 201-233. doi:10.1017/s0140525x00078407

Neely, C. B., \& LeCompte, D. C. (1999). The importance of semantic similarity to the irrelevant speech effect. Memory \& Cognition, 27, 37-44. doi:10.3758/bf03201211

Neely, J. H. (1991). Semantic priming effects in visual word recognition: A selective review of current findings and theories. In D. Besner \& G. W. Humphreys (Eds.), Basic processes in reading: Visual word recognition (pp. 264-336), Hillsdale, NJ: Lawrence Erlbaum Associates. doi:10.4324/9780203052242

Neely, J. H., \& Kahan, T. A. (2001). Is semantic activation automatic? A critical re-evaluation. In H. L. Roediger III, J. S. Nairne, I. Neath, \& A. M. Surprenant (Eds.), Science conference series. The nature of remembering: Essays in honor of Robert G. Crowder (pp. 69-93). Washington, DC, US: American Psychological Association. doi:10.1037/10394-005

Neumann, O. (1984). Automatic processing: A review of recent findings and a plea for an old theory. In W. Prinz \& A. F. Sanders (Eds.), Cognition and motor processes (pp. 255293). Berlin, Germany: Springer-Verlag. doi:10.1007/978-3-642-69382-3_17

Neumann, O. (1990). Direct parameter specification and the concept of perception. Psychological Research, 52, 207-215. doi:10.1007/BF00877529

New, B., Pallier, C., Brysbaert, M., \& Ferrand, L. (2004). Lexique 2 : A new French lexical database. Behavior Research Methods, Instruments, \& Computers, 36, 516-524. doi:10.3758/BF03195598 
Nöstl, A., Marsh, J. E., \& Sörqvist, P. (2012). Expectations modulate the magnitude of attentional capture by auditory events. PLoS ONE 7(11), e48569. doi:10.1371/journal.pone.0048569

Parmentier, F. B. R. (2008). Towards a cognitive model of distraction by auditory novelty: The role of involuntary attention capture and semantic processing. Cognition, 109, 345-362. doi:10.1016/j.cognition.2008.09.005

Parmentier, F. B. R., Elford, G., Escera, C., Andrés, P., \& San Miguel, I. (2008). The cognitive locus of distraction by acoustic novelty in the cross-modal oddball task. Cognition, 106, 408-432. doi:10.1016/j.cognition.2007.03.008

Parmentier, F. B. R., Elsley, J. V., Andrés, P., \& Barceló, F. (2011). Why are auditory novels distracting? Contrasting the roles of novelty, violation of expectation and stimulus change. Cognition, 119, 374-380. doi:10.1016/j.cognition.2011.02.001

Parmentier, F. B. R., \& Hebrero, M. (2013). Cognitive control of involuntary distraction by deviant sounds. Journal of Experimental Psychology: Learning, Memory, and Cognition, 39, 1635-1641. doi:10.1037/a0032421

Parmentier, F. B. R., \& Kefauver, M. (2015). The semantic aftermath of distraction by deviant sounds: Crosstalk interference is mediated by the predictability of semantic congruency. Brain Research, 1626, 247-257. doi:10.1016/j.brainres.2015.01.034

Parmentier, F. B. R., Turner, J., \& Perez, L. (2014). A dual contribution to the involuntary semantic processing of unexpected spoken words. Journal of Experimental Psychology: General, 143, 38-45. doi:10.1037/a0031550

Peer, P., Emeršič, Ž., Bule, J., Žganec-Gros, J., \& Štruc, V. (2014). Strategies for exploiting independent cloud implementations of biometric experts in multibiometric scenarios. Mathematical Problems in Engineering, 2014, 585139. doi:10.1155/2014/585139 
Posner, M. I., \& Snyder, C. R. (1975). Attention and cognitive control. In R.L. Solso (Ed.), Information processing and cognition. Hillsdale, NJ: Erlbaum.

Ratcliff, R., \& McKoon, G. (1988). A retrieval theory of priming in memory. Psychological Review, 95, 385-408. doi:10.1037//0033-295x.95.3.385

Rivenez, M., Darwin, C. J., \& Guillaume, A. (2006). Processing unattended speech. Journal of the Acoustical Society of America, 119, 4027-4040. doi:10.1121/1.2190162

Röer, J. P., Bell, R., \& Buchner, A. (2013). Self-relevance increases the irrelevant sound effect: Attentional disruption by one's own name. Journal of Cognitive Psychology, 25, $925-$ 931. doi:10.1080/20445911.2013.828063

Röer, J. P., Bell, R., \& Buchner, A. (2015). Specific foreknowledge reduces auditory distraction by irrelevant speech. Journal of Experimental Psychology: Human Perception and Performance, 41, 692-702. doi:10.1037/xhp0000028

Röer, J. P., Bell, R., Körner, U., \& Buchner, A. (2019). A semantic mismatch effect on serial recall: Evidence for interlexical processing of irrelevant speech. Journal of Experimental Psychology: Learning, Memory, and Cognition, 45, 515-525. doi:10.1037/xlm0000596

Röer, J. P., Körner, U., Buchner, A., \& Bell, R. (2017a). Attentional capture by taboo words: A functional view of auditory distraction. Emotion, 17, 740-750. doi:10.1037/emo0000274

Röer, J. P., Körner, U., Buchner, A., \& Bell, R. (2017b). Semantic priming by irrelevant speech. Psychonomic Bulletin \& Review, 24, 1205-1210. doi:10.3758/s13423-016-1186-3

Roye, A., Jacobsen, T., \& Schröger, E. (2007). Personal significance is encoded automatically by the human brain: an event- related potential study with ringtones. European Journal of Neuroscience, 26, 784-790. doi:10.1111/j.1460-9568.2007.05685.x 
Schneider, W., \& Shiffrin, R. M. (1977). Controlled and automatic human information processing: I. Detection, search, and attention. Psychological Review, 84, 1-66. doi:10.1037/0033-295X.84.1.1

Schnuerch, R., Kreitz, C., Gibbons, H., \& Memmert, D. (2016). Not quite so blind: Semantic processing despite inattentional blindness. Journal of Experimental Psychology: Human Perception and Performance, 42, 459-463. doi:10.1037/xhp0000205

Schröger, E. (1997). On the detection of auditory deviations: A pre-attentive activation model. Psychophysiology, 34, 245-257. doi:10.1111/j.1469-8986.1997.tb02395.x

Schröger, E., Bendixen, A., Trujillo-Barreto, N. J., \& Roeber, U. (2007). Processing of abstract rule violations in audition. PLoS ONE 2(11), e1131. doi:10.1371/journal.pone.0001131

Schröger, E., \& Wolff, C. (1998). Behavioral and electrophysiological effects of task-irrelevant sound change: A new distraction paradigm. Cognitive Brain Research, 7, 71-87. doi:10.1016/S0926- 6410(98)00013-5

Shelton, J. T., Elliott, E. M., Eaves, S. D., \& Exner, A. L. (2009). The distracting effects of a ringing cell phone: An investigation of the laboratory and the classroom setting. Journal of Environmental Psychology, 29, 513-521. doi:10.1016/j.jenvp.2009.03.001

Smyth, M. M., Hay, D. C., Hitch, G. J., \& Horton, N. J. (2005). Serial position memory in the visual-spatial domain: Reconstructing sequences of unfamiliar faces. Quarterly Journal of Experimental Psychology, 58A, 909-930. doi:10.1080/02724980443000412

Sokka, L., Huotilainen, M., Leinikka, M., Korpela, J., Henelius, A., Alain, C., Müller, K., \& Pakarinen, S. (2014). Alterations in attention capture to auditory emotional stimuli in job burnout: An event-related potential study. International Journal of Psychophysiology, 94, 427-436. doi:10.1016/j.ijpsycho.2014.11.001 
Sokolov, E. N. (1963). Perception and the conditioned reflex. London, England: Pergamon Press.

Sörqvist, P. (2010). High working memory capacity attenuates the deviation effect but not the changing-state effect: Further support for the duplex-mechanism account of auditory distraction. Memory \& Cognition, 38, 651-658. doi:10.3758/MC.38.5.651

Sörqvist, P., Dahlström, Ö., Karlsson, T., \& Rönnberg, J. (2016). Concentration: The neural underpinnings of how cognitive load shields against distraction. Frontiers in Human Neuroscience, 10, 122. doi:10.3389/fnhum.2016.00221

Sörqvist, P., \& Marsh, J. E. (2015). How concentration shields against distraction. Current Directions in Psychological Science, 24, 267-272. doi:10.1177/0963721415577356

Sörqvist, P., Marsh, J. \& Jahncke, H. (2010). Hemispheric asymmetries in auditory distraction. Brain and Cognition, 74, 79-87. doi:10.1016/j.bandc.2010.06.007

Sörqvist, P., Stenfelt, S. \& Rönnberg, J. (2012). Working memory capacity and visual-verbal cognitive load modulate auditory-sensory gating in the brainstem: Toward a unified view of attention. Journal of Cognitive Neuroscience, 24, 2147-2154. doi:10.1162/jocn_a_00275

Surprenant, A. M., Neath, I., \& Brown, G. D. A. (2006). Modeling age-related differences in immediate memory using SIMPLE. Journal of Memory and Language, 55, 572-586. doi:10.1016/j.jml.2006.08.001

Sussman, E., Winkler, I., \& Schröger, E. (2003). Top-down control over involuntary attention switching in the auditory modality. Psychonomic Bulletin \& Review, 10, 630-637. doi:10.3758/BF03196525 
Theeuwes, J., \& Burger, R. (1998). Attentional control during visual search: The effect of irrelevant singletons. Journal of Experimental Psychology: Human Perception and Performance, 24, 1342-1353. doi:10.1037//0096-1523.24.5.1342

Thierry, G., \& Roberts, M. V. (2007). Event-related potential study of attention capture by affective sounds. Neuroreport, 18, 245-248. doi:10.1097/WNR.0b013e328011dc95

Tse, C.-S., \& Neely, J. H. (2007). Semantic and repetition priming effects for Deese/RoedigerMcDermott (DRM) critical items and associates produced by DRM and unrelated study lists. Memory \& Cognition, 35, 1047-1066. doi:10.3758/BF03193477

Underwood, G., \& Everatt, J. (1996). Automatic and controlled information processing: The role of attention in the processing of novelty. In O. Neumann \& A. F. Sanders (Eds.), Handbook of perception and action, Vol. 3. Attention (pp. 185-227). San Diego, CA: Academic Press. doi:10.1016/S1874-5822(96)80023-5

Vachon, F., Hughes, R. W., \& Jones, D. M. (2012). Broken expectations: Violation of expectancies, not novelty, captures auditory attention. Journal of Experimental Psychology: Learning, Memory, and Cognition, 38, 164-177. doi:10.1037/a0025054

Vachon, F., \& Jolicœur, P. (2011). Impaired semantic processing during task-set switching: Evidence from the N400 in rapid serial visual presentation. Psychophysiology, 48, 102111. doi:10.1111/j.1469-8986.2010.01040.x

Vachon, F., \& Jolicœur, P. (2012). On the automaticity of semantic processing during task switching. Journal of Cognitive Neuroscience, 24, 611-626.doi:10.1162/jocn_a_00149

Vachon, F., Labonté, K., \& Marsh, J. E. (2017). Attentional capture by deviant sounds: A noncontingent form of auditory distraction? Journal of Experimental Psychology: Learning, Memory, and Cognition, 43, 622-634. doi:10.1037/xlm0000330 
Vachon, F., Tremblay, S., \& Jones, D. M. (2007). Task-set reconfiguration suspends perceptual processing: Evidence from semantic priming during the attentional blink. Journal of Experimental Psychology: Human Perception and Performance, 33, 330-347. doi:10.1037/0096-1523.33.2.330

West, G. L., Anderson, A. A. K., \& Pratt, J. (2009). Motivationally significant stimuli show visual prior entry: Evidence for attentional capture. Journal of Experimental Psychology: Human Perception and Performance, 35, 1032-1042. doi:10.1037/a0014493

Winkler, I., Denham, S. L., \& Nelken, I. (2009). Modeling the auditory scene: Predictive regularity representations and perceptual objects. Trends in Cognitive Sciences, 13, 532540. doi:10.1016/j.tics.2009.09.003

Woldorff, M. G., Hackley, S. A., \& Hillyard, S. A. (1991). The effects of channel-selective attention on the mismatch negativity wave elicited by deviant tones. Psychophysiology, 28, 30-42. doi:10.1111/j.1469-8986.1991.tb03384.x

Wood, N., \& Cowan, N. (1995). The cocktail party phenomenon revisited: How frequent are attention shifts to one's name in an irrelevant auditory channel? Journal of Experimental Psychology: Learning, Memory, and Cognition, 21, 255-260. doi:10.1037//02787393.21 .1 .255

Yi, D. J., Woodman, G. F., Widders, D., Marois, R., \& Chun, M. M. (2004). Neural fate of ignored stimuli: Dissociable effects of perceptual and working memory load. Nature Neuroscience, 7, 992-996. doi:10.1038/nn1294 


\section{Appendix A}

\section{Analysis of the acoustical and phonological properties of the spoken alphanumerical stimuli used in Experiments 1, 3A, 4, 5, 6, and 7}

In order to ascribe any disruption caused by the insertion of a spoken letter within a sequence of spoken digits or of a spoken digit among spoken letters to the semantic properties of the auditory stimuli, we need to rule out the possibility that such an effect is of acoustical nature. To do so, we contrasted the acoustical and phonological qualities of the two sets of spoken alphanumerical stimuli used in Experiments 1, 3A, 4, 5, 6, and 7. For each of the nine digits (19) and the nine letters (B, F, H, K, M, Q, R, X, and Z), we extracted 10 variables relating to their duration, loudness, spectral composition, and phonological characteristics.

The acoustic parameters were evaluated by Praat software (Boersma \& Weenink, 2019), using the standard extraction settings. The duration corresponds to the time (in ms) from the onset to the offset of the waveform of a spoken item. Although all sound files were edited to last $250 \mathrm{~ms}$, some spoken items were slightly shorter than that. In order to determine whether one set of stimuli was systematically louder, we extracted the overall intensity (in $\mathrm{dB}$ ) of the sound as well as the root mean square or RMS amplitude (in Pa). Spectral composition was assessed through four parameters (all expressed in $\mathrm{Hz}$ ): $F 0$ (i.e. the mean fundamental frequency), $F 0$ range (computed by subtracting the maximum $\mathrm{F} 0$ from the minimum $\mathrm{F} 0$ ), and the frequency of the first two formants (F1 and $F 2$ ) since they are particularly important in speech recognition.

The phonological parameters were extracted from the lexical database for French Lexique (version 3.83; New, Pallier, Brysbaert, \& Ferrand, 2004). We focused the phonological analysis on three variables: the number of syllables, the number of phonemes, and the phonological uniqueness point (PUP), which refers to the point in a word at which it becomes unique from all 
other words in the lexicon. It is noteworthy that some letters were absent from the Lexique database. For some of them, the phonological variables could be extrapolated by using the values for words with the exact same phonology (e.g., the word 'aime' for the letter 'M' or the word 'hache' for the letter ' $H$ '). This could not be done, however, for the letter ' $F$ '. This letter was therefore excluded from the phonological analysis.

The results of the acoustical and phonological analyses of the two sets of alphanumerical stimuli are presented in Table A1. This table details the mean, standard deviation, minimum and maximum of each variable separately for the digit set and the letter set. We compared the mean of each parameter between the two types of stimulus using independent-sample $t$-tests and found no significant differences $(p s>.11)$. It is clear from these results that there was no systematic discrepancy at either acoustical or phonological level between the spoken letters and the spoken digits employed in the present study that could account for any disruption produced by the introduction of a categorical deviant item within a sequence of spoken alphanumerical stimuli. 
Table A1

Results of the acoustical/phonological analysis of the spoken alphanumerical stimuli

\begin{tabular}{|c|c|c|c|c|c|c|c|c|c|}
\hline & \multicolumn{4}{|c|}{ Digits $(n=9)$} & \multicolumn{4}{|c|}{ Letters $(n=9)$} & \multirow[b]{2}{*}{$t^{\mathrm{a}}$} \\
\hline & $M$ & $S D$ & Min & Max & $M$ & $S D$ & Min & Max & \\
\hline Duration (ms) & 246.4 & 5.9 & 235 & 250 & 244.8 & 10.37 & 220 & 250 & 0.42 \\
\hline \multicolumn{10}{|l|}{$\begin{array}{l}\text { Loudness } \\
\text { analysis }\end{array}$} \\
\hline Intensity (dB) & 75.8 & 1.2 & 74.2 & 78.2 & 76.1 & 1.2 & 74.3 & 77.6 & -0.52 \\
\hline RMS amp. (Pa) & 0.105 & 0.016 & 0.087 & 0.139 & 0.111 & 0.013 & 0.092 & 0.128 & -0.78 \\
\hline \multicolumn{10}{|l|}{$\begin{array}{l}\text { Spectral } \\
\text { analysis }(\mathrm{Hz})\end{array}$} \\
\hline F0 & 116.3 & 2.9 & 113.2 & 122.6 & 114.5 & 1.3 & 112.4 & 117.2 & 1.67 \\
\hline F0 range & 30.2 & 22.8 & 6.9 & 80.2 & 36.0 & 12.0 & 15.6 & 48.6 & -0.67 \\
\hline F1 & 541.8 & 143.9 & 333 & 777 & 599.9 & 221.1 & 317 & 953 & -0.66 \\
\hline F2 & 1793.6 & 248.5 & 1421 & 2228 & 1840.3 & 286.0 & 1500 & 2310 & -0.37 \\
\hline \multicolumn{10}{|l|}{$\begin{array}{l}\text { Phonological } \\
\text { analysis }^{\text {b }}\end{array}$} \\
\hline No. syllables & 1.00 & 0 & 1 & 1 & 1.00 & 0 & 1 & 1 & - \\
\hline No. phonemes & 2.89 & 0.93 & 1 & 4 & 2.33 & 0.50 & 2 & 3 & 1.38 \\
\hline PUP & 2.89 & 0.93 & 1 & 4 & 2.33 & 0.50 & 2 & 3 & 1.38 \\
\hline
\end{tabular}

Note. RMS amp. = root mean square of the amplitude; F0 = Fundamental frequency; F1 = First formant; F2 = Second formant; PUP = phonological uniqueness point

${ }^{a}$ No $t$-test was significant $(p s>.11)$.

bPhonological attributes were unavailable for the letter ' $\mathrm{F}$ '. 


\section{Appendix B}

\section{Analysis of the acoustical and phonological properties of the spoken category exemplars of}

\section{Experiment 2A}

In order to ascertain that any categorical deviation effect that could be found with spoken category exemplars employed in Experiment 2A is not of acoustical nature, we applied to those auditory stimuli the same acoustical/phonological analysis we performed on spoken alphanumerical items (see Appendix A for more details). To be more specific, we contrasted the acoustical and phonological properties of the deviant exemplar of each deviant sequences to those of the standard exemplars composing the remaining of these sequences. This allowed to determine whether the deviant exemplars were special cases in terms of acoustical and phonological qualities. Using Praat software (Boersma \& Weenink, 2019), we extracted the seven acoustical parameters (see Appendix A) for the 12 deviant spoken words encountered in the experiment and for the 16 standard spoken words of each of the four stimulus sets used in this experiment (i.e. animals, tools, fruits, and occupations). The three phonological parameters were extracted from the Lexique database (New et al., 2004) for all but one standard exemplar ('infirmière'), absent from the database.

First, we compared the acoustical and phonological parameters of the set of deviant exemplars to those of each of the four sets of standard exemplars. The results are presented in Table B1. We compared the mean of each parameter between deviants and standards from each of the four categories employed using independent-sample $t$-tests. We found no significant differences for three categories, namely animals ( $p \mathrm{~s}>.08)$, tools $(p \mathrm{~s}>.12)$, and fruits ( $p \mathrm{~s}>.17)$. Such results suggested that the spoken exemplars used as deviants overall shared similar acoustical and phonological properties with the spoken exemplars from these three categories. 


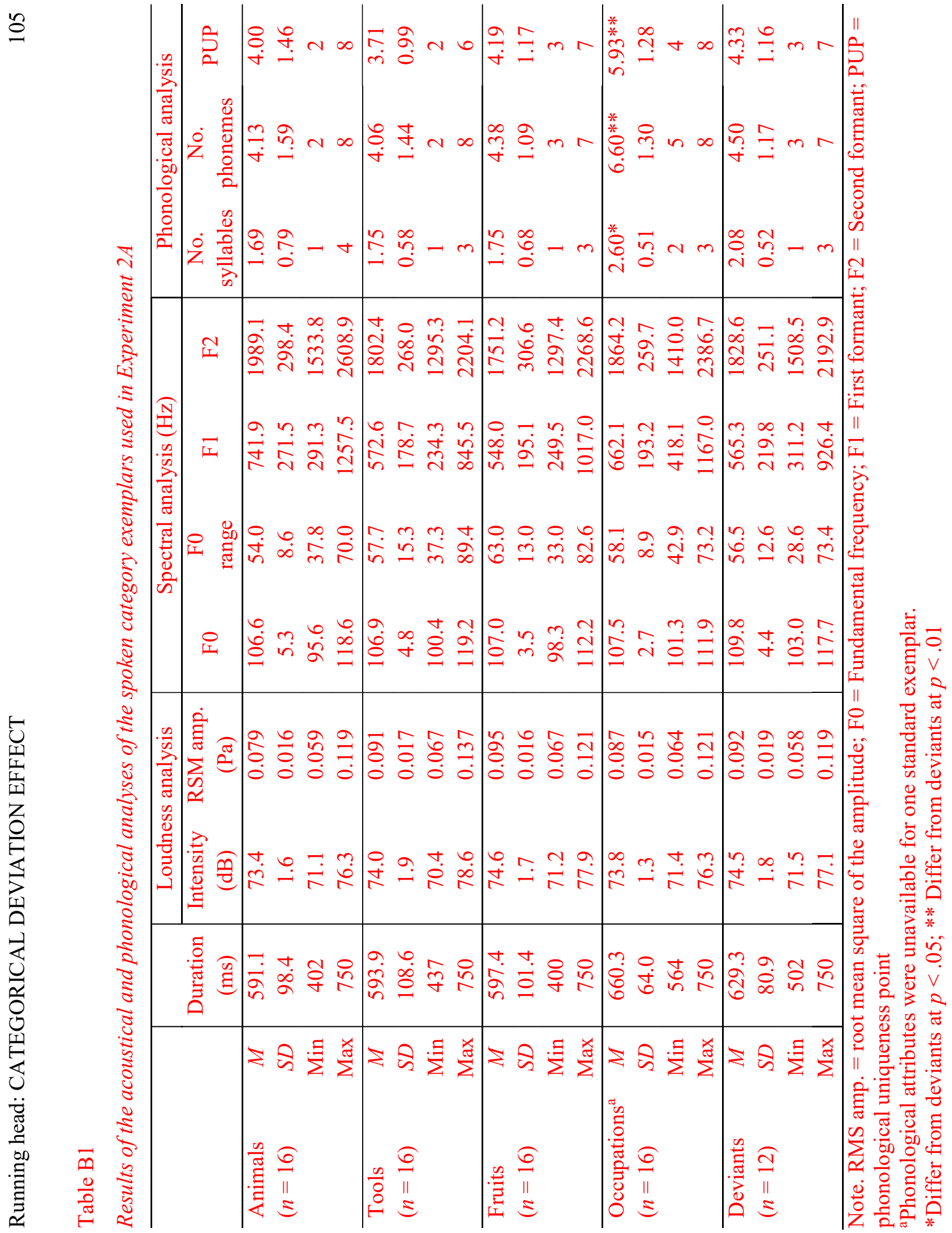


The analysis revealed, however, that the set of occupation exemplars appeared, overall, phonologically more complex than the set of deviant exemplars. In fact, the former showed, on average, significantly more syllables and phonemes and a significantly greater PUP than the latter. No difference was found at the acoustical level between the two sets ( $p \mathrm{~s}>.11)$.

One could argue that despite the overall acoustical and phonological similarities between the deviants and the standards (at least for 3 out of the 4 categories), it is nevertheless conceivable that each deviant exemplar stood out from the rest of the auditory sequence at the acoustical and/or phonological levels. The analysis performed on the values averaged on all stimuli would not capture this possibility. As a consequence, for each category, we computed separately for each of the 12 sequences the absolute difference from the sequence mean of each extracted parameter for the deviant exemplar as well as for the standard exemplar with the largest deviation from the mean. This allowed us to determine whether the randomly selected deviant exemplars were particularly acoustically and/or phonologically salient. Table B2 presents the averaged absolute mean difference for both the 12 deviants and the 12 standards of each category with the largest mean difference. The results revealed that the deviant exemplars did not depart from the rest of the sequence on any of the parameters analyzed. This was true for all categories, including occupations.

Again, one could argue that averaging the deviations from the mean over all deviant exemplars could preclude from knowing whether each individual deviant really stood out from the other sounds composing the sequence in which it was embedded. We therefore took a more 'individual' approach and computed for each deviant the number of parameters for which it showed the largest deviation from the sequence mean. An acoustically/phonologically salient deviant item should depart from the rest of the auditory sequence on several parameters. Table $\mathrm{B} 3$ presents the distribution of deviant sequences as a function of the number of parameters for 


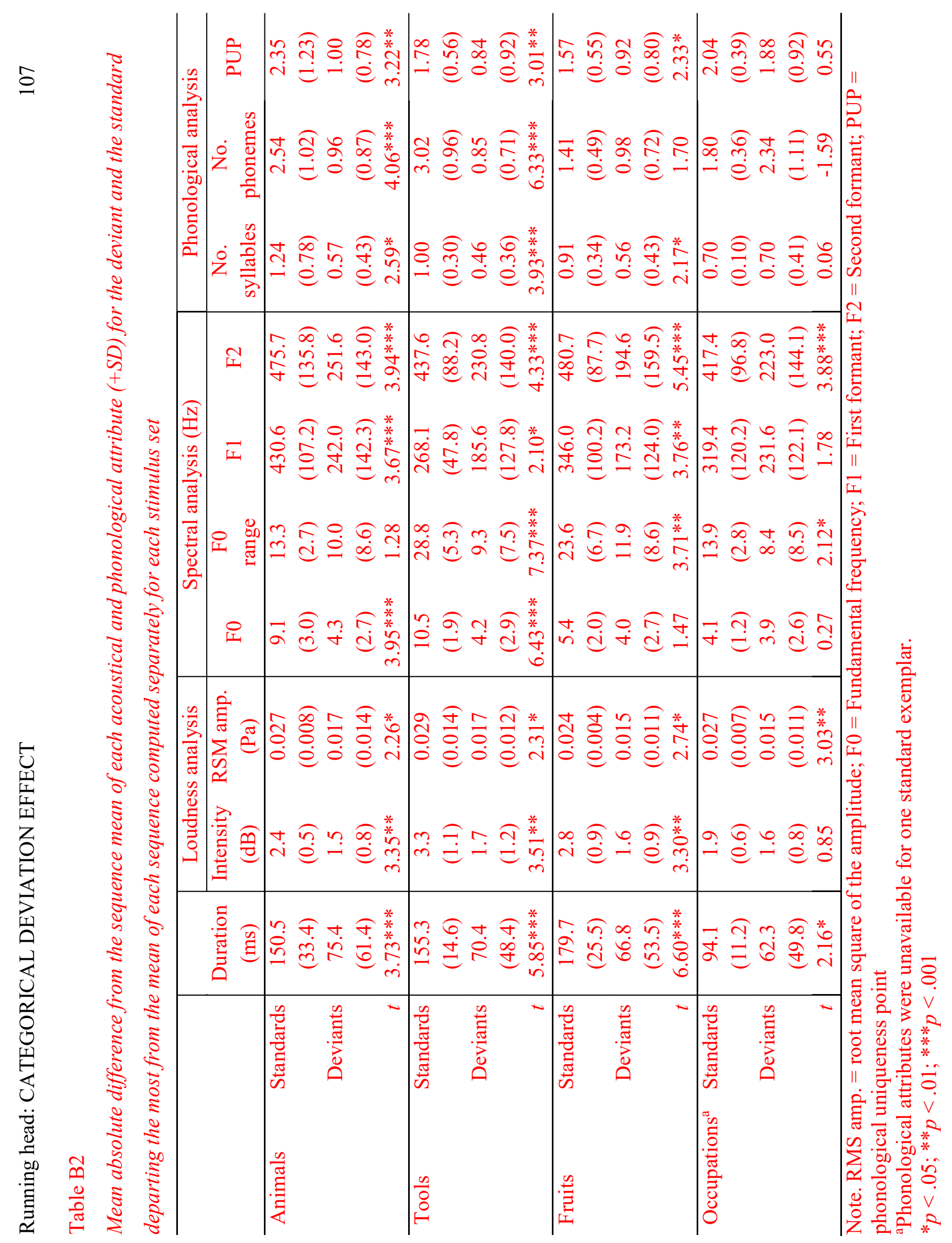


which the deviant exemplar stood out from the other (standard) exemplars of the sequence for each of the four categories. This table shows that for the first three categories (i.e., animals, tools and fruits), the deviant had the highest deviation from the mean on two or less parameters in 10 out of the 12 sequences. Such results indicated that deviant exemplars were not acoustical and/or phonological special cases when inserted among animal, tool, or fruit exemplars. The portrait was different, however, with the set of occupation exemplars as almost half deviants departed from the rest of the sequence on four or more acoustical/phonological properties. This suggests that some deviant exemplars could be considered as acoustical and/or phonological special cases when embedded among occupation exemplars.

\section{Table B3}

Number of auditory sequences (out of 12) in which the categorical deviant had the largest deviation from the mean on one or more acoustical or phonological parameters for each stimulus set

\begin{tabular}{lcccccc}
\hline & \multicolumn{6}{l}{ No. of parameters with the largest deviation from the mean for the deviant } \\
\cline { 2 - 6 } & 0 & 1 & 2 & 3 & 4 & $5+$ \\
\hline Animals & 2 & 4 & 4 & 2 & 0 & 0 \\
Tools & 5 & 0 & 5 & 2 & 0 & 0 \\
Fruits & 3 & 7 & 0 & 2 & 0 & 0 \\
Occupations & 2 & 3 & 0 & 2 & 3 & 2 \\
\hline
\end{tabular}

Overall, the analysis of the acoustical and phonological features of the spoken stimuli used in Experiment 2A revealed a proper matching of standard and deviant exemplars for 3 out of 4 categories. The phonological mismatch between occupation and deviant exemplars has therefore been taken into account in the analysis of the categorical deviation effect. 


\section{Appendix C}

\section{Analysis of the acoustical and phonological properties of the spoken category exemplars of}

\section{Experiment 2B}

To determine whether acoustical factors could contribute to any categorical deviation effect found in Experiment 2B, we applied to the spoken exemplars employed in Experiment 2B the same acoustical/phonological analysis we performed on the auditory stimuli of Experiment 2A (see Appendix B for more details). More specifically, we contrasted the acoustical and phonological properties of the deviant exemplar of each of the 12 auditory sequences to those of the standard exemplars composing the remaining of these sequences. This allowed to determine whether the deviant exemplars were special cases in terms of acoustical and phonological qualities. Using Praat software (Boersma \& Weenink, 2019), we extracted the seven acoustical parameters (see Appendix A) for 12 deviant spoken words and 81 standard spoken words (three exemplars appeared in two different deviant trials). The three phonological parameters (see Appendix A) were extracted from the Lexique database (New et al., 2004) for all but two standard exemplars ('tilapia' and 'Baileys'), absent from the database.

We first contrasted the average value of each parameters between the two types of spoken items. The results are presented in Table C1. We compared the mean of each parameter between deviants and standards using independent-sample $t$-tests and found no significant difference ( $p \mathrm{~s}>$ .21). Such results suggested that the spoken exemplars used as deviants overall shared similar acoustical and phonological properties with the other spoken exemplars that were surrounding them. 
Table C1

Results of the acoustical and phonological analyses of the spoken category exemplars used in Experiment $2 B$

\begin{tabular}{|c|c|c|c|c|c|c|c|c|c|}
\hline & \multicolumn{4}{|c|}{ Standards $(n=81)$} & \multicolumn{4}{|c|}{ Deviants $(n=12)$} & \multirow[b]{2}{*}{$t^{\mathrm{a}}$} \\
\hline & $M$ & $S D$ & Min & $\operatorname{Max}$ & $M$ & $S D$ & Min & $\operatorname{Max}$ & \\
\hline Duration (ms) & 664.8 & 39.0 & 402 & 750 & 681.1 & 77.4 & 535 & 750 & -0.65 \\
\hline \multicolumn{10}{|l|}{$\begin{array}{l}\text { Loudness } \\
\text { analysis }\end{array}$} \\
\hline Intensity (dB) & 73.7 & 0.9 & 68.2 & 77.2 & 73.9 & 1.7 & 71.3 & 76.9 & -0.25 \\
\hline RMS amp. (Pa) & 0.091 & 0.010 & 0.048 & 0.138 & 0.092 & 0.015 & 0.067 & 0.117 & -0.26 \\
\hline \multicolumn{10}{|l|}{$\begin{array}{l}\text { Spectral } \\
\text { analysis }(\mathrm{Hz})\end{array}$} \\
\hline F0 & 107.9 & 3.5 & 98.9 & 124.0 & 109.0 & 4.8 & 101.9 & 118.5 & -0.67 \\
\hline F0 range & 61.2 & 13.2 & 29.0 & 316.7 & 59.3 & 15.1 & 28.5 & 83.5 & 0.33 \\
\hline F1 & 594.1 & 104.5 & 265 & 1189 & 563.3 & 174.7 & 311 & 828 & 0.52 \\
\hline $\mathrm{F} 2$ & 1892.4 & 112.4 & 1279 & 2609 & 1853.6 & 232.9 & 1460 & 2193 & 0.52 \\
\hline \multicolumn{10}{|l|}{$\begin{array}{l}\text { Phonological } \\
\text { analysis }^{\mathrm{b}}\end{array}$} \\
\hline No. syllables & 1.99 & 0.28 & 1 & 4 & 2.25 & 0.75 & 1 & 4 & -1.13 \\
\hline No. phonemes & 4.94 & 0.47 & 2 & 9 & 5.58 & 1.68 & 3 & 9 & -1.29 \\
\hline PUP & 4.48 & 0.41 & 2 & 8 & 4.92 & 2.07 & 3 & 8 & -0.71 \\
\hline
\end{tabular}

Note. RMS amp. = root mean square of the amplitude; F0 = Fundamental frequency; F1 = First formant; F2 = Second formant; PUP = phonological uniqueness point ${ }^{a}$ No $t$-test was significant $(p s>.21)$.

${ }^{b}$ Phonological attributes were unavailable for two standard exemplars.

We then tested whether deviant exemplars stood out from the rest of the auditory sequence at the acoustical and/or phonological levels by computing separately for each of the 12 sequences the absolute difference from the sequence mean of each extracted parameter for both the deviant exemplar and the standard exemplar with the largest deviation from the mean. This 
allowed to determine whether the randomly selected deviant exemplars were particularly acoustically and/or phonologically salient. Table C2 presents the averaged absolute mean difference for both the 12 deviants and the 12 standards with the largest mean difference. The results revealed that the deviant exemplars did not depart from the rest of the sequence on any of the parameters analyzed.

Table C2

Mean absolute difference from the sequence mean of each acoustical and phonological attribute (+SD) for the deviant and the standard departing the most from the mean of each sequence

\begin{tabular}{rccc}
\hline & Standards & Deviants & $t$ \\
\hline Duration (ms) & $146.0(53.1)$ & $84.4(54.6)$ & $2.81^{* *}$ \\
\hline Loudness analysis & & & \\
Intensity (dB) & $2.7(1.0)$ & $1.3(0.8)$ & $3.73^{* * *}$ \\
RMS amp. (Pa) & $0.025(0.010)$ & $0.011(0.006)$ & $4.07^{* * *}$ \\
Fpectral analysis $\quad$ Fz) & $7.9(3.1)$ & $2.6(1.5)$ & $5.30^{* * *}$ \\
F0 range (Hz) & $40.7(57.6)$ & $13.6(11.1)$ & 1.60 \\
F2 (Hz) & $312.0(129.7)$ & $145.5(131.6)$ & $3.12^{* *}$ \\
No. syllables & $405.3(133.4)$ & $223.0(121.9)$ & $3.50^{* *}$ \\
No. phonemes & $2.40(0.86)$ & & $2.73^{*}$ \\
PUP & $2.13(0.73)$ & $1.36(1.09)$ & $2.60^{*}$ \\
\hline
\end{tabular}

Note. RMS amp. $=$ root mean square of the amplitude; F0 = Fundamental frequency; F1 $=$ First formant; F2 = Second formant; PUP = phonological uniqueness point ${ }^{*} p<.05 ; * * p<.01 ; * * * p<.001$

As in Appendix B, we also took a more 'individual' approach and computed for each deviant the number of parameters for which it showed the largest deviation from the sequence 
mean. An acoustically/phonologically salient deviant item should depart from the rest of the auditory sequence on several parameters. Table $\mathrm{C} 3$ presents the distribution of deviant sequences as a function of the number of parameters for which the deviant exemplar stood out from the other (standard) exemplars. This table shows that the deviant had the highest deviation from the mean on one or no parameter in half of the sequences. Moreover, among the three times the deviant stood out on 4 out of the 10 parameters, the deviant had the largest deviation on both the number of phonemes and the PUP on two occasions. Given these two phonological properties are highly correlated, it could be assumed that the deviant stood out on four parameters only once. Thus, there is no clear evidence from this individual analysis that the deviant exemplars were acoustical and/or phonological special cases.

\section{Table C3}

Number of auditory sequences (out of 12) in which the categorical deviant had the largest deviation from the mean on one or more acoustical or phonological parameters

\begin{tabular}{lclllllc}
\hline & \multicolumn{6}{l}{ No. of parameters with the largest deviation from the mean for the deviant } \\
\cline { 2 - 7 } & 0 & 1 & 2 & 3 & 4 & $5+$ \\
\hline No. of sequences & 3 & 3 & 1 & 2 & 3 & 0 \\
\hline
\end{tabular}

Overall, the analysis of the acoustical and phonological qualities of the spoken exemplars used in Experiment 2B provided no evidence that the disruption of serial recall could be attributed to the particular acoustical/phonological properties of the deviant exemplars. 


\section{Appendix D}

\section{Analysis of the acoustical and phonological properties of the spoken category exemplars of}

\section{Experiment 3B}

We performed an acoustical/phonological analysis of the exemplars used in Experiment 3B (see Appendices B and C for more details) akin to that undertaken for the spoken exemplars employed in Experiment 2. We contrasted the acoustical and phonological properties of the deviant exemplar of each of the 16 auditory sequences to those of the standard exemplars composing the remaining of these sequences to determine whether the deviants were special cases in terms of acoustical and phonological qualities. More specifically, we extracted seven acoustical parameters using Praat software (Boersma \& Weenink, 2019; see Appendix A) and three phonological parameters from the Lexique database (New et al., 2004; see Appendix B) for the 8 deviant exemplars and the 12 standard exemplars used in each of the two blocks of trials.

We first contrasted the average value of each parameters between the deviant and standard exemplars separately for the 'animals' and 'tools' blocks. The results are presented in Table D1. We compared the mean of each parameter between deviants and standards from both blocks employed using independent-sample $t$-tests. We found no significant differences in both 'animals' block $(p \mathrm{~s}>.10)$ and 'tools' block $(p \mathrm{~s}>.19)$, suggesting that the spoken exemplars used as deviants overall shared similar acoustical and phonological properties with the spoken exemplars in both blocks of trials.

We then tested, for each block, whether deviant exemplars stood out from the rest of the auditory sequence at the acoustical and/or phonological levels by computing separately for each of the 8 sequences the absolute difference from the sequence mean of each extracted parameter for both the deviant exemplar and the standard exemplar with the largest deviation from the 


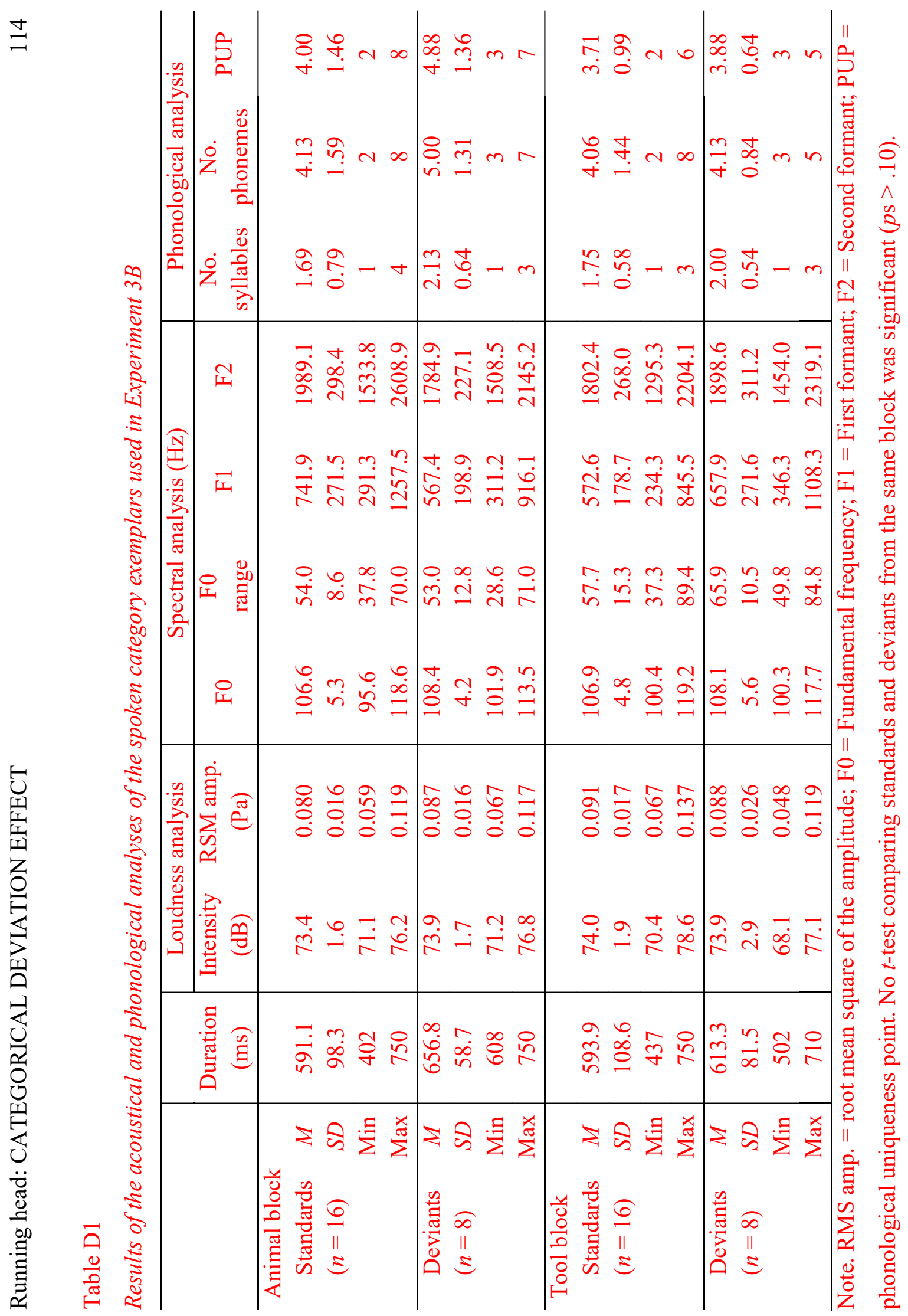


mean. This allowed us to determine whether the randomly selected deviant exemplars were particularly acoustically and/or phonologically salient. Table D2 presents the averaged absolute mean difference for both the 8 deviants and the 8 standards with the largest mean difference in each block. The results revealed that the deviant exemplars from both the 'animals' and 'tools' blocks did not depart from the rest of the sequence on any of the parameters analyzed.

As in Appendices B and C, we also took a more 'individual' approach and computed for each deviant the number of parameters for which it showed the largest deviation from the sequence mean. An acoustically/phonologically salient deviant item should depart from the rest of the auditory sequence on several parameters. Table D3 presents the distribution of deviant sequences as a function of the number of parameters for which the deviant exemplar stood out from the other (standard) exemplars. This table shows that the deviant had the highest deviation from the mean on two or less parameters in $75 \%$ of the 'animals' sequences and $87.5 \%$ of the 'tools' sequences. Thus, there is no clear evidence from this individual analysis that the deviant exemplars were acoustical and/or phonological special cases.

Overall, the analysis of the acoustical and phonological qualities of the spoken exemplars used in Experiment /B provided no evidence that the disruption of serial recall could be attributed to the particular acoustical/phonological properties of the deviant exemplars. 
Table D2

Mean absolute difference from the sequence mean of each acoustical and phonological attribute $(+S D)$ for the deviant and the standard departing the most from the mean of each sequence computed separately for each block of trials

\begin{tabular}{|c|c|c|c|c|c|c|}
\hline & \multicolumn{3}{|c|}{ Animal block } & \multicolumn{3}{|c|}{ Tool block } \\
\hline & Standards & Deviants & $t$ & Standards & Deviants & $t$ \\
\hline Duration & 154.1 & 62.8 & $3.36^{* *}$ & 157.0 & 76.5 & $6.51 * * *$ \\
\hline (ms) & $(38.7)$ & $(66.5)$ & & $(15.7)$ & $(31.3)$ & \\
\hline \multicolumn{7}{|c|}{ Loudness analysis } \\
\hline Intensity & 2.4 & 1.5 & 1.91 & 3.5 & 2.3 & 1.60 \\
\hline$(\mathrm{dB})$ & $(0.5)$ & $(1.2)$ & & $(1.1)$ & (1.7) & \\
\hline RMS amp. & 0.028 & 0.013 & $3.03 * *$ & 0.030 & 0.022 & 1.20 \\
\hline$(\mathrm{Pa})$ & $(0.007)$ & $(0.012)$ & & $(0.016)$ & $(0.013)$ & \\
\hline \multicolumn{7}{|c|}{ Spectral analysis $(\mathrm{Hz})$} \\
\hline \multirow{4}{*}{ F0 range } & 9.4 & 3.8 & $3.89 * *$ & 10.9 & 4.5 & $4.75 * * *$ \\
\hline & $(3.0)$ & (2.7) & & $(1.8)$ & (3.3) & \\
\hline & 13.7 & 8.7 & 1.42 & 31.6 & 11.6 & $5.53 * * *$ \\
\hline & $(2.4)$ & $(9.7)$ & & (3.2) & $(9.7)$ & \\
\hline \multirow[t]{2}{*}{$\mathrm{F} 1$} & 430.9 & 226.5 & $3.22 * *$ & 265.7 & 218.4 & 0.77 \\
\hline & $(81.0)$ & $(160.1)$ & & $(54.7)$ & $(165.3)$ & \\
\hline \multirow[t]{2}{*}{$\mathrm{F} 2$} & 524.8 & 233.3 & $3.86^{* *}$ & 456.87 & 287.6 & $2.82 *$ \\
\hline & (127.3) & (171.6) & & $(20.5)$ & (168.5) & \\
\hline \multicolumn{7}{|c|}{ Phonological analysis } \\
\hline No. & 1.34 & 0.61 & $2.35^{*}$ & 0.86 & 0.50 & $2.20 *$ \\
\hline syllables & $(0.79)$ & $(0.39)$ & & $(0.37)$ & $(0.28)$ & \\
\hline No. & 2.80 & 1.13 & $3.56^{* *}$ & 2.64 & 0.63 & $4.65 * * *$ \\
\hline phonemes & $(0.89)$ & (0.99) & & (1.16) & $(0.40)$ & \\
\hline \multirow[t]{2}{*}{ PUP } & 2.66 & 1.27 & $2.66^{*}$ & 1.51 & 0.53 & $3.67 * *$ \\
\hline & (1.14) & $(0.95)$ & & $(0.70)$ & $(0.28)$ & \\
\hline
\end{tabular}

Note. RMS amp. = root mean square of the amplitude; F0 = Fundamental frequency; F1 = First formant; F2 = Second formant; PUP = phonological uniqueness point $* p<.05 ; * * p<.01 ; * * * p<.001$ 
Table D3

Number of auditory sequences (out of 8) in which the categorical deviant had the largest deviation from the mean on one or more acoustical or phonological parameters for each block of trials

No. of parameters with the largest deviation from the mean for the deviant

\begin{tabular}{llllllc}
\cline { 2 - 6 } & 0 & 1 & 2 & 3 & 4 & $5+$ \\
\hline Animal block & 3 & 3 & 0 & 1 & 1 & 0 \\
Tool block & 3 & 1 & 3 & 0 & 1 & 0 \\
\hline
\end{tabular}

